\title{
Community structure and dynamics of wood-rotting Basidiomycetes on decomposing conifer trunks in northern Finland
}

\author{
PERTTI RENVALL
}

\begin{abstract}
Renvall, P. 1995: Community structure and dynamics of wood-rotting Basidiomycetes on decomposing conifer trunks in northern Finland. - Karstenia 35:1-51. Helsinki. ISSN 0453-3402

The succession and organization of wood-rotting Basidiomycetes, as indicated by their fruit body production, were studied on naturally fallen, decomposing trunks of Picea abies (L.) Karsten subsp. obovata (Ledeb.) Domin and Pinus sylvestris L. in northeastern Finland. The study area consists of northern boreal primeval forests that show no signs of forestry practices or wood utilization. Altogether 120 species of Basidiomycetes were found on Picea and 104 on Pinus. The species compositions varied with the following characteristics of the trunks: stage of decay, history of fungal infections preceding the tree fall, diameter and amount of bark. The structures of fungal communities were analysed by using DCA ordination and the divisive clustering technique TWINSPAN. The results indicate that wood-inhabiting fungi succeed each other according to a regular order and that they differ from each other in their association with microclimatic regimes, in their strategies in resource capture, in their competition ability during the wood decomposition, and in their species associates. Physical and chemical properties of the host tree species and the microclimate of the growth site govern the basic trends in the community development of wood-inhabiting fungi. The first steps in tree trunk decomposition greatly depend on the way the tree died. Primary decayers affect the composition of the fungi at later stages of decay, by opening successional pathways for specific groups of saprotrophs. It is concluded that the conservation of lignicolous fungi can succeed only after comprehensive analysis of fungal community development and the achievement of a thorough understanding of the decomposition dynamics of fallen tree trunks.
\end{abstract}

Key words: brown rot, charred wood, community ecology, conservation, microclimate, Picea, Pinus, primeval forest, succession, threatened fungi, white rot, wood-rotting fungi

Pertti Renvall, Department of Ecology and Systematics, P.O. Box 47, FIN-00014 University of Helsinki, Finland

\section{Introduction}

Decaying wood is an essential ecological component in forest ecosystems. Snags, stumps, branches and fallen tree trunks, in particular, harbour a large number of fungi and insects, even birds and some mammals. Furthermore, fallen extensively decayed trunks serve as a substrate for bryophytes and other plants, and play an active role in forest regeneration as seedbeds of trees (e.g., Swift 1977, Harmon et al. 1986, Franklin et al. 1987, Harmon \& Franklin 1989). Species richness of a single forest is closely linked with the dynamics of the wood decomposition in the area (cf. Kotiranta \& Niemelä 1981, Maser \& Trappe 1984, Söderström 1988, Andersson \& Hytteborn 1991, Hansson 1992, Angelstam \& Mikusiński 1994, Kaila et al. 1994, Siitonen 1994, Virkkala et al. 1994).

The decomposition of wood and the development of decomposer communities has traditionally been regarded as one of the most clear-cut cases of succession. The term substratum succession was used by Park (1968) for the succession of fungi that 
occurs on any colonizable plant or animal substrate. Frankland (1992) preferred the term resource succession, adding, however, that in practice a fallen tree trunk is 'a heterogeneous complex of closely interrelated resources, usually exhibiting a pattern of decomposers related to the distribution of different tissues'. An even more dynamic concept was introduced by Boddy (1992), who rejected the term succession as too simplistic and described the development of fungal communities in wood as $a$ complex, multidimensional process which follows a diverse array of 'optional' pathways. In this paper I use the term succession in a broad sense to comprise the general temporal dynamics of fungi during the decomposition of wood (internal succession in fallen trees, see Maser \& Trappe 1984). The term itself is used without any particular connection to equilibrium and nonequilibrium concepts in ecology (Chesson \& Case 1986, DeAngelis \& Waterhouse 1987, Giller \& Gee 1987). The term fungal community is understood as a highly dynamic assemblage of decomposer fungi that changes continuously in space and time (see Swift 1987).

The decomposition of a fallen tree trunk is a complex biological process which usually starts within the living tree. Bacteria and ascomycetes are often reported to be the first invaders of fresh, intact wood (Roll-Hansen \& Roll-Hansen 1979, Eriksson et al. 1990, Solheim 1992a, b, Hallaksela \& SalkinojaSalonen 1992, Hallaksela 1993), but actively growing trees may also be troubled by more effective decayers (see Boddy \& Rayner 1983b, Rayner \& Boddy 1988a), often by certain polypores. These fungi act as pathogens of living trees and are of economic significance in that they substantially reduce the quality and quantity of commercial timber (e.g., Stenlid 1993). Soon after the death and fall of a tree it will be invaded by a variety of saprotrophic fungi, especially Basidiomycetes. Basidiomycetes occupy the whole tree trunk and make up the majority of organisms responsible for the wood decay (Montgomery 1982, Swift 1982, Rayner \& Boddy 1988a). In the course of time the decomposing trunk undergoes structural and chemical changes, and the primary rotters become replaced by more competitive decomposers. Finally, more or less ephemeral fungi utilize the last remnants of rotten wood; the fallen tree trunk will be totally decomposed and the remnants incorporated in humus.

As beautifully shown by Rayner, Boddy and their co-workers (Boddy \& Rayner 1983c, Coates \& Rayner 1985c, Boddy et al. 1987, Rayner et al. 1987, Chapela \& Boddy 1988a, b, c, Rayner \& Boddy 1988b, Griffith \& Boddy 1990, Boddy 1993), wood-inhabiting fungi make up both temporally and spatially changing populations and communities, and the population and community structure and development are based on dynamic interactions of species and individuals (genets). Although the basic biochemical and physiological pathways in wood decomposition, and the general patterns of mycelial dynamics in wood are fairly well known in vitro (Rayner \& Boddy 1988a, Eriksson et al. 1990), quantitative data on mycelial dynamics, populations and communities is available for just a few species of wood-inhabiting fungi, and most of it for managed forests of the temperate zone. Accordingly, despite the rapid methodological progress in ecological research (Nobles 1948, 1965, Rypáček 1966, Korhonen 1978a, b, Stalpers 1978, Rayner \& Todd 1979, Todd \& Rayner 1980, Chase \& Ullich 1983, Stenlid 1985, Chamuris \& Falk 1987, Lewis \& Hansen 1991, Kay \& Vilgalys 1992, Smith et al. 1992, Karlsson 1993) the major portion of the spatial and temporal patterns and processes of wood fungi are waiting to be described.

The decomposition of wood in nature, tree-fall dynamics, and the subsequent changes in the species composition of wood-rotting fungi have received surprisingly little attention. Most studies dealing with the communities or populations of saprotrophic fungi (see Boddy 1992) have focused on the onset of the wood decomposition, and the data have predominantly been obtained from experiments carried out at least partially under unnatural conditions. Although tree death, as an ecological process, is a well-recognized biological phenomenon (Graham 1925, McCullough 1948, Muhle \& Leblanc 1978, Maser \& Trappe 1984, Söderström 1987, Jonsson 1993), studies on the succession of wood-rotting fungi have been undertaken chiefly in managed forests, and often the data pertain to branches and twigs or merely cut stumps (Käärik \& Rennerfelt 1957, Meredith 1959, 1960, Butcher 1968, Rayner 1977a, b, Runge 1978, 1986, Lopez 1983, Chapela et al. 1988, Hintikka 1993). Both mycofloristically and temporally comprehensive investigations on the dynamics of fungi on fallen, decaying tree trunks (Jahn 1962, 1966, 1968, Lange 1986, 1992, Luschka 1993) are very few.

Northern regions of Finland, Norway and Sweden have long escaped forestry and land use. Even now, patches of naturally regenerating virgin forests are found in these regions. However, during the last decades intensive forestry practices, including clearcutting and artificial regeneration of tree species, have invaded also these regions, and the number of unmanaged forests has drastically diminished even in northern Fennoscandia. The remaining pristine or near- 
pristine forests have become more and more fragmented and are now almost always isolated by vast areas of managed forests (Hämet-Ahti 1983, Karström 1992a, b, Hansson 1992, Haila et al. 1994). Furthermore, because modern forestry eradicates fallen tree trunks, forests with a continuity of natural decomposition have been widely destroyed, and many fungi that prefer old-forest habitats have diminished in number and been classified as threatened in Nordic countries (Bendiksen \& Høiland 1992, Rassi et al. 1992, Kotiranta \& Niemelä 1993, Hallingbäck 1994).

Our knowledge of the dynamics of wood fungi in the boreal zone has at the same time remained scanty. Saprotrophic fungal communities, particularly at late stages of the wood decomposition, are still extremely poorly known. Numerous regional species lists have been published on boreal wood-inhabiting fungi, and their host species preferences are generally well known in northern Europe (see Eriksson 1958, Eriksson \& Strid 1969, Strid 1975, Johansen \& Ryvarden 1977, Hjortstam \& Johannesen 1980, Kotiranta \& Niemelä 1981, Hjortstam 1981, Erkkilä \& Niemelä 1986, Larsson 1986, Aandstad \& Ryvarden 1987, Renvall et al. 1991b, Mathiassen 1993, Ryvarden 1994). By contrast, information on the population and community ecology of these fungi is so far almost exclusively available as descriptive data. The community development and spatial structures and the forest-fire dynamics of wood-rotting fungi in primeval forests, as well as the detailed effects of forestry on the composition of such fungi, are still poorly known.

The purpose of my study was four-fold: 1) to investigate the dynamics of wood-rotting Basidiomycetes on fallen, decomposing trunks of Picea abies (L.) Karsten subsp. obovata (Ledeb.) Domin and Pinus sylvestris $\mathrm{L}$. in an undisturbed northern boreal primeval forest, 2) to describe temporal patterns and processes of fungal communities on decaying conifers and to outline the successional pathways of such fungi, 3) to analyse, how these fungi organize themselves in trunks of different sizes and 4) to offer comprehensive data for nature conservation purposes.

\section{Materials and methods}

Sampling and notes on the study area

The field work was done in 1987-1992 (excluding 1990) in the Värriö Strict Nature Reserve and in Urho Kekkonen National Park, northeastern Finland (Fig. 1). Most of the material was collected in August and September, but to avoid bias due to the seasonality of fungal basidiocarps, the field work in 1988 was begun at the end of July and continued until early October.

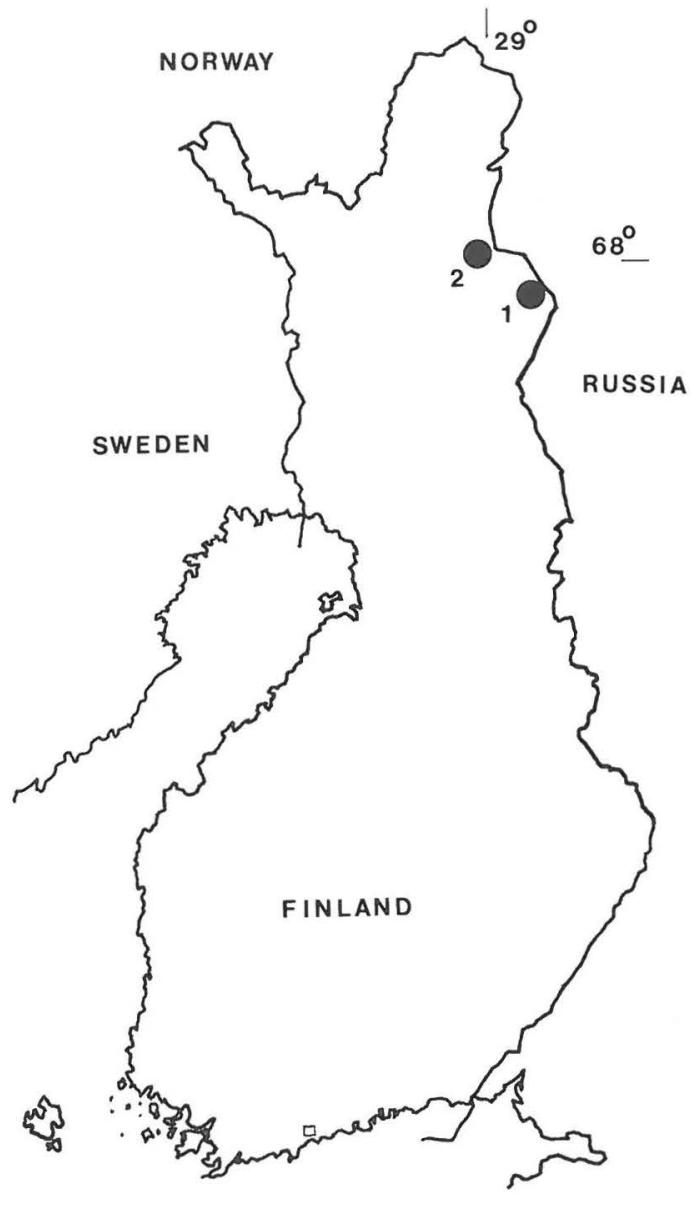

Fig. 1. The location of the study areas in Finland: 1 Värriö Strict Nature Reserve, 2 Urho Kekkonen National Park.

The study area is covered by primeval, naturally regenerating northern boreal (in the sense of Ahti et al. 1968) forests. The forests are characterized by abundant fallen and decaying trunks and natural stumps, which have not been touched by forestry. The pristine state of the area and the great number of decaying trunks at different stages of wood decomposition offered me a unique opportunity to study the natural dynamics of wood-rotting fungi. The main forest site types are briefly described below. For a more detailed description of the location, geology, climate and the vegetation of the study area see Renvall et al. (1991a).

The Scots pine (Pinus sylvestris) and Siberian spruce (Picea abies subsp. obovata) make up the majority of the woody vegetation in the area. There are two main types of dry pinedominated forests in the area. The very dry pine woodlands on sandy soils can be referred to the Uliginosum-VacciniumEmpetrum site type (UVET, Kalela 1961). Pine forests which are a little moister than the UVET and occur mostly on more fertile till soils belong to the Empetrum-Myrtillus type (EMT). 
Although single pine emergents may grow in more mesic sites, intermixed with spruce, the pine material derives exclusively from these two types of dry pine-dominated forests. The elevation of the pine forests studied varied from $190 \mathrm{~m}$ to $360 \mathrm{~m}$. Most of the data on pine trunks was collected in the Värriö Strict Nature Reserve, but fresh windfalls, in particular, were also studied in the Urho Kekkonen National Park. As shown by Zackrisson (1977), forest fires have played an essential role in the regeneration dynamics of boreal forests. The fire scars, which were fairly evenly distributed on standing pine trees and stumps, and the charred pine trunks lying on the ground, are evidence of repeated fires and indicate the strong impact of fire on the forest ecology.

Spruce predominates on moister till soils, where it forms open and well-illuminated stands. These naturally sparse mesic spruce forests on the lower slopes of fjelds belong to the Hylocomium-Myrtillus type (HMT). The more or less closed woodlands extend up to an altitude of approximately 450 metres. Spruce trunks were studied only in the Värriö Reserve, at an altitude of 280-400 metres. Climatical conditions are much more stable in these forests than in the pine-dominated forests. The moss layer is thick, dense and almost continuously mesic, which evidently has efficiently reduced the frequency of forest fires. Although spruce charcoal particles may be found in the soil, there are no visible signs of fire, e.g., fire scars or charred trunks, on trees in the spruce forests of the study area, indicating that wildfires are less common in spruce-dominated forests than in adjacent pine forests.

The spruce forests are denser and richer in brook ravines and in the valleys of small rivers, especially on soils affected by spring flooding, than they are on the lower slopes of fjelds. Tree growth is better and the trees are taller and usually thicker than in pure forests of the HMT, and the stands are more shaded. Most of these brookside forests maintain a lush grass - herb undergrowth, and evidently they have seldom, if ever, been disturbed by fires. These forests are characterized by a distinctly humid and stable microclimate, and although they usually form only narrow belts 5 to $30 \mathrm{~m}$ wide along the brooks, I treat them as a distinct forest type (here referred to as the Geranium-Dryopteris-Myrtillus site type, GDMT, of Kalela 1961).

To summarize, the major forest types differ from each other in soil properties, floristic compositions and the architecture of the woody vegetation. They are characterized by specific microclimatical conditions and exhibit differencies in their history of forest fire dynamics.

In order to study the effects of wood decomposition on the dynamics of wood-inhabiting fungi, an equal number of fallen trunks at different stages of decomposition were examined. Fifty trunks of pine at each decay stage $1-4$ were studied in UVET and EMT forests $(50 \times 4 \times 2)$, and 50 trunks of spruce at decay stages $1-4$ were studied in HMT forests $(50 \times 4 \times 1)$. In addition, 20 extensively decayed trunks or their remnants (decay stage 5) were examined for each forest type. In brookside spruce forests (GDMT) the restricted number of freshly fallen trunks allowed only 20 trunks per decay stage to be studied. Altogether, I carefully examined
440 trunks of pine and 320 trunks of spruce. Fifty-one $(11.6 \%)$ of the pine trunks were strongly charred. Because the trunks at different stages of decomposition were for the most part unevenly distributed in the forests, sample plots were not established; rather, the sample trunks were subjectively selected. However, six voucher plots $(25 \mathrm{~m} \times$ $25 \mathrm{~m}$ ) were established in selected stands of spruce in the Värriö Reserve. Fallen spruce trunks on these plots were numbered and marked permanently with plastic labels for later identification and study.

The sample trunks had to fulfil the following requirements: 1) they had to be naturally fallen, i.e. either uprooted in a storm or broken because of decay or by heavy snow, 2) they had to be lying on the ground; trunks already broken but still leaning on adjacent trees or big rocks were passed over, 3) the base diameter had to exceed $10 \mathrm{~cm}, 4$ ) the length of the trunk had to exceed $1.5 \mathrm{~m}, 5$ ) the trunks had to lie inside closed forests, i.e., at least 50 metres away from mires or other extensive treeless and microclimatically strongly deviating areas.

All Basidiomycetes (sporocarps) fruiting on these trunks were identified in situ or collected for later identification. An observation of rhizomorphs was considered adequate for ascertaining the presence of Piloderma croceum (P. Karst.) Jülich (incl. $P$. olivaceum (Parmasto) Hjortstam) in a trunk. The specimens of the fungi were identified by the author, except for tomentelloid fungi (the genera Tomentella (Pers.) Pat., Tomentellopsis Hjortstam, Pseudotomentella Svrček), which were determined by Urmas Kõljalg. Sterile specimens of corticioid fungi (e.g. the genera Botryobasidium Donk and Tubulicrinis Donk) and four rotten and unidentified specimens of agarics were excluded from the material. For a description of the microscopical examination see Renvall \& Niemelä (1992a). Voucher specimens are preserved in the Botanical Museum of the University of Helsinki $(\mathrm{H})$.

In addition to the fungal species compositions on the trunks, the following characteristics of each trunk were recorded or measured: 1) decay stage, 2) base diameter (not $\mathrm{DBH}$ !; bark included, measured to the nearest $5 \mathrm{~cm}$ ), 3) length (measured to the nearest $0.5 \mathrm{~m}$ ), 4) type of stem breakage (uprooted with root plate; stump+trunk; fallen because of root rot; snow break), 5) amount of bark (in \%, estimated to the nearest $5 \%$ ), 6) diameter where basidiocarps of a certain species are found (measured to the nearest $5 \mathrm{~cm}$ ), 7) primary species composition and approximate amount of epiphytic bryophytes and lichens. Because standing trees were sparsely and fairly evenly distributed in the study area under each forest site type the canopy cover was not estimated in this study.

To obtain more data on the preferences of the fungi for other trunk characteristics (diameter, amount of bark) I examined 28 additional pine trunks and 29 additional spruces. As well, I studied 27 spruce trunks in stunted forests of spruce swamps, and these were included in the numerical treatment of the material (DECORANA, TWINSPAN) together with the rest of the data. Altogether, then, the material studied comprised 468 pine and 376 spruce trunks. 


\section{Decay classification}

I used a five-point scale (see Renvall \& Niemelä 1994) to classify sample trunks into different stages of decay (abbreviated as D.S.). The scale corresponds fairly well with the classification presented by Muhle and LeBlanc (1975), Sollins (1982), or Maser and Trappe (1984), although the tree species (and the size and turnover time of the trunks) are different. The main character used in defining the decay stage was the hardness of wood. A single classification scale could be used because spruce and pine trees do not differ markedly from each other in their wood density and initial hardness. The decay stage was tested by sticking a knife (with a $10 \mathrm{~cm}$ long, $2 \mathrm{~cm}$ wide and $2.5 \mathrm{~mm}$ thick blade) several times into different parts of the trunk. The crown usually differs in decay stage from the basal part of the trunk, and the top third of the tree was therefore excluded from the analysis of hardness. The decay stage classification that was used is presented in Table 1.
Annual variability in the fruit-body formation is one of the main problems in quantitative fungal ecology, especially when methods are based on observations of basidiocarps (Vogt et al. 1992). I tried to avoid the problem in the present study by expanding the periods of field work to cover several growing seasons and by excluding the material that was collected during the least productive growing season. In 1987 both temperature and precipitation during the growing season were very low, and the fruit body formation of certain polypores (see Renvall et al. 1991b) and most of the terrestrial agarics was minimal. Accordingly the material collected that year was totally excluded from the numerical analyses (DECORANA, TWINSPAN) of the material. However, it was included in the analysis of species preferences for different trunk characteristics.

The moisture content of wood rises steadily during the decomposition (Griffin 1977, Lambert et al. 1980, Dix 1985, Sollins et al. 1987). In order to evaluate the decay classification used, and to estimate the changes in the moisture content, I took wood samples from selected fallen

Table 1. The classification used for dividing the trunks of Picea abies ssp. obovata and Pinus sylvestris into different stages of wood decomposition.

(D.S.) and freshly broken, but still undecayed trunks belong here. Epiphytic flora chiefly the same as on standing and still living trees.

Wood fairly hard; knife penetrates ca. 1-2 cm into the wood. Pine trunks usually already decorticated or with only small patches of bark left, often with patches of epiphytic lichens (e.g. Parmeliopsis ambigua, P. hyperopta). Bark on spruce starting to break up and small patches of epixylic cryptogams may already be found.

3 Wood fairly soft, undergoing an intense process of decomposition, small area of wood already decomposed, but especially the upper part of the trunk usually with distinctly harder parts; knife penetrates fairly easily ca. $3-5 \mathrm{~cm}$ into the wood. When lifted the crown of the trunk usually breaks off. Pine trunks usually decorticated and at least partly covered with cup lichens (Cladonia spp.). Spruce trunks usually still partly corticated, but naked parts already covered by a variety of epixylic lichens and bryophytes, in continuously mesic localities by Ptilidium pulcherrimum, Lophozia spp., Cephalozia spp. and many other hepatics.

4 Wood soft; the whole blade of the knife easily penetrates into the wood. Trunks extensively decayed and, usually, large sections of the wood usually completely decomposed. When lifted the trunk easily falls apart. Usually without bark or only small patches left; extensively covered by bryophytes and lichens, some of them typical forest ground inhabitants, e.g., Dicranum spp., reindeer lichens (Cladonia spp.). Sometimes also covered with Vaccinium vitis-idaea, Empetrum nigrum or Linnaea borealis.

Wood very soft, almost completely decomposed and disintegrates easily down between fingers. The whole trunk (or its remnants) considerably shrunken and its outer surface difficult to determine, being usually almost totally covered with ground floor cryptogams (e.g., reindeer lichens, Dicranum spp., Hylocomium splendens, Pleurozium schreberi) and/or dwarf shrubs. The remnants of spruce trunks often bearing seedlings of spruce. 
trunks at different stages of decomposition. The sampling was done in the Värriö Reserve at the end of August 1991 and 1992, and covered three forest site types (HMT, UVET, EMT). Ten samples each from spruce and pine representing different stages of decomposition were taken from the basal parts of middle-sized (base diameter 25$40 \mathrm{~cm}$ ) trunks. The size of the sample was 50-100 cubic $\mathrm{cm}$. Because rain water affects the water content of wood, especially in trunks at late stages of decomposition, the sampling was done after a period of three rainless days. Before taking the samples I first removed the bark and outernmost layer $(0.5-1.5 \mathrm{~cm})$ of the wood. The samples were immediately sealed in plastic bags and stored at $\mathrm{c}$. $+5^{\circ} \mathrm{C}$. Wet masses of the samples were measured within a week of the sampling. After that, samples were dried at $55^{\circ} \mathrm{C}$ and reweighed. Moisture content ( $\%$ of wet mass) was calculated as (wet mass-dry mass) $\times 100 /$ wet mass. In total I analysed 50 spruce and 50 pine samples.

\section{Data analysis}

For the detection of ecologically related groups of fungi and mycofloristically similar groups of sample trunks, the processed data matrixes (presence/absence) of Picea and Pinus were subjected separately to DCA (Detrended Correspondence Analysis) using the program DECORANA (Hill \& Gauch 1980). This method arranges both species and trunks along axes so that those which are most similar to each other are closest. In addition, the divisive clustering technique TWINSPAN (Two-way Indicator Species Analysis; Hill 1979) was applied. TWINSPAN is based on a reciprocal algorithm, and divisions of the trunks were made on the basis of the presence or absence of fungi. In the analyses, species with less than three observations were excluded. Tomentelloid fungi were treated under the collective name Tomentella sp., and Piloderma olivaceum was included in $P$. croceum.

To compare the similarity of the species compositions of the fungal communities between trunks at different stages of decay, Sørensen's Quotient of Similarity (Q.S.) (Magurran 1988) was applied. The index was calculated with the formula

$$
\text { Q.S. }=2 \mathrm{c} / \mathrm{a}+\mathrm{b}
$$

$\mathrm{a}=$ total number of species on trunks at decay stage a $\mathrm{b}=$ total number of species on trunks at decay stage $\mathrm{b}$ $\mathrm{c}=$ number of species common on trunks at decay stages $\mathrm{a}$ and $\mathrm{b}$

A special index of Relative Locality (R.L.) was used to describe the average proximal vs. distal location of the species (mycelia) on sample trunks. The index was calculated with the formula

$$
\text { R.L. }=\left(f_{1} / b_{1}+f_{2} / b_{2}+\ldots+f_{n} / b_{n}\right) / n
$$

$\mathrm{f}=$ trunk diameter where basidiocarps of a certain species are found (mean of the values on a single trunk) $\mathrm{b}=$ base diameter of the trunk

$\mathrm{n}=$ number of trunks
The R.L. index, which gives a simplified numerical expression for the optimal lengthwise distribution of a species, obtains values $<1$. High values $(0.8-1)$ indicate that the species prefers basal parts of the sample trunks, while low values $(<0.6)$ indicate a preference for the crown or the top third of the trunk.

\section{Nomenclature}

The nomenclature of polypores mostly follows Ryvarden and Gilbertson (1993, 1994). However, the genera Amyloporia Bondartsev \& Singer and Antrodia P. Karsten are treated according to Niemelä (1994a) and the divisions of the genera Oligoporus Bref. and Postia Fr. according to Renvall (1992). The work of Eriksson et al. (1973 and later volumes) served as the basis for the identification and nomenclature of the corticiaceous fungi. However, the species names in the families Lachnocladiaceae D.A. Reid and Coniophoraceae Ulbr. are according to Ginns (1978) and Hallenberg (1985a). The names of the Gloeocystidiellaceae Jülich are according to Ginns and Freeman (1994), and the nomenclature of the genus Hypochnicium J. Eriksson is after Hallenberg (1985b). The names of tomentelloid fungi were adopted from Stalpers (1993), and the names of other aphyllophoroid fungi follow Jülich (1984). The nomenclature of gilled fungi is according to Hansen and Knudsen (1992).

The trees and shrubs of the area are described in Hämet-Ahti et al. (1992) and the names of the other vascular plants are listed in Hämet-Ahti et al. (1986). The nomenclature of bryophytes follows Koponen et al. (1977) and that of lichens Santesson (1993). The author names of plants and lichens can be found in the above papers, and they are not repeated here.

\section{Results}

\section{Characteristics of sample trunks}

The size of the sample trunks varied with the tree species and forest site type. The variability of the base diameter and the length of the sample trunks are reported in Tables 2 and 3. Differences in the soil fertility and other growth conditions between the four forest site types are reflected in the size of the trees. In both spruce and pine trunks the base diameter ranged from 10 to $90 \mathrm{~cm}$, but the mean base diameter of spruce trunks was $40 \mathrm{~cm}$ and that of pine trunks $31 \mathrm{~cm}$. The base was thicker than $50 \mathrm{~cm}$ in $13.4 \%$ of the spruce trunks and $5.9 \%$ of the pine trunks. Spruce trunks found in rich brookside forests (GDMT) were, on average, thicker (mean base diameter $45 \mathrm{~cm}$ ) and longer than those found in mesic spruce forests (HMT; mean base diameter $38 \mathrm{~cm}$; Table 2), and 
pine trunks on more mesic sites (EMT) were somewhat bigger (mean base diameter $34 \mathrm{~cm}$ ) than those found on dry and sandy soil (UVET; mean base diameter $29 \mathrm{~cm}$; Table 3 ).

During decomposition, a fallen conifer trunk loses at least part of its bark before the wood becomes soft. In the present material the amount of bark on sample trunks exhibited a strong negative correlation with the stage of decay, although there was strong variation within each stage (Fig. 2). A fallen spruce trunk retains its bark for a fairly long time, whereas uprooted pine trunks become decorticated sooner, approximately within 10-20 years after falling. However, most of the pine trunks studied were already decorticated when they fell. The moisture

Table 2. Background information on fallen spruce (Picea abies subsp. obovata) trunks. The base diameter distribution is arranged according to stage of decay (1-5) and forest site type.

\begin{tabular}{|c|c|c|c|c|c|c|c|c|c|c|c|c|c|c|c|c|}
\hline \multirow{3}{*}{$\begin{array}{l}\text { Base diam } \\
\quad(\mathrm{cm})\end{array}$} & \multicolumn{16}{|c|}{ Decay stage } \\
\hline & & 1 & & & 2 & & & 3 & & & 4 & & & 5 & & \\
\hline & HMT & GDMT & tot. & HMT & GDMT & tot. & HMT & GDMT & tot. & HMT & GDMT & tot. & HMT & GDMT & tot. & $\mathrm{n}$ \\
\hline $10-20$ & 5 & 2 & 7 & - & - & - & 5 & 2 & 7 & 2 & - & 2 & 2 & - & 2 & 18 \\
\hline $25-35$ & 23 & 2 & 25 & 29 & 7 & 36 & 16 & 6 & 22 & 12 & 4 & 16 & 11 & 6 & 17 & 116 \\
\hline $40-50$ & 20 & 12 & 32 & 18 & 8 & 26 & 23 & 6 & 29 & 26 & 12 & 38 & 5 & 13 & 18 & 143 \\
\hline $55-$ & 2 & 4 & 6 & 3 & 5 & 8 & 6 & 6 & 12 & 10 & 4 & 14 & 2 & 1 & 3 & 43 \\
\hline $\mathrm{b}$ & 35 & 43 & 37 & 37 & 49 & 41 & 37 & 46 & 41 & 44 & 47 & 45 & 35 & 39 & 37 & \\
\hline 1 & 11.1 & 15.6 & 12.4 & 10.6 & 13 & 11.3 & 10.9 & 13.7 & 11.7 & 10.4 & 11 & 10.6 & 8.8 & 10.3 & 9.6 & \\
\hline $\mathrm{n}$ & 50 & 20 & 70 & 50 & 20 & 70 & 50 & 20 & 70 & 50 & 20 & 70 & 20 & 20 & 40 & 320 \\
\hline
\end{tabular}

HMT = Hylocomium-Myrtillus type

GDMT = Geranium-Dryopteris-Myrtillus type

$\mathrm{b}=$ mean of the base diameter.

$\mathrm{l}=$ mean of the length $(\mathrm{m})$

$\mathrm{n}=$ number of trunks

Table 3. Background information on fallen pine (Pinus sylvestris) trunks. The base diameter distribution is arranged according to stage of decay (1-5) and forest site type.

\begin{tabular}{|c|c|c|c|c|c|c|c|c|c|c|c|c|c|c|c|c|}
\hline \multirow{3}{*}{$\begin{array}{l}\text { Base diam } \\
\quad(\mathrm{cm})\end{array}$} & \multicolumn{16}{|c|}{ Decay stage } \\
\hline & & 1 & & & 2 & & & 3 & & & 4 & & & 5 & & \\
\hline & UVET & EMT & tot. & UVET & EMT & tot. & UVET & EMT & tot. & UVET & EMT & tot. & UVET & EMT & tot. & $\mathrm{n}$ \\
\hline $10-20$ & 19 & 6 & 25 & 10 & 8 & 18 & 11 & 8 & 19 & 12 & 10 & 22 & 5 & 6 & 11 & 95 \\
\hline $25-35$ & 23 & 26 & 49 & 26 & 19 & 45 & 22 & 20 & 42 & 30 & 22 & 52 & 15 & 14 & 29 & 217 \\
\hline $40-50$ & 6 & 13 & 19 & 12 & 15 & 27 & 16 & 17 & 33 & 8 & 15 & 23 & - & - & - & 102 \\
\hline $55-$ & 2 & 5 & 7 & 2 & 8 & 10 & 1 & 5 & 6 & - & 3 & 3 & - & - & - & 26 \\
\hline $\mathrm{b}$ & 29 & 36 & 32 & 31 & 35 & 33 & 30 & 36 & 33 & 27 & 32 & 30 & 26 & 30 & 28 & \\
\hline 1 & 14.6 & 13.6 & 14.1 & 10.1 & 9.3 & 9.7 & 7 & 10.5 & 8.8 & 5.3 & 7.4 & 6.4 & 7 & 5.5 & 6.3 & \\
\hline $\mathrm{n}$ & 50 & 50 & 100 & 50 & 50 & 100 & 50 & 50 & 100 & 50 & 50 & 100 & 20 & 20 & 40 & 440 \\
\hline $\mathrm{c}$ & - & - & - & 4 & 4 & 8 & 9 & 5 & 14 & 21 & 8 & 29 & - & - & - & 51 \\
\hline
\end{tabular}

UVET = Uliginosum-Vaccinium-Empetrum type

$\mathrm{EMT}=$ Empetrum-Myrtillus type

$\mathrm{b}=$ mean of the base diameter.

$\mathrm{l}=$ mean of the length $(\mathrm{m})$

$\mathrm{n}=$ number of trunks

$\mathrm{c}=$ number of strongly charred trunks 
content of wood in the sample trunks increased sharply with the stage of decay (Fig. 3), but there was considerable variation in the moisture content within each decay stage. For both spruce and pine the variation was greatest at decay stage 3, indicating that the sample trunks at that stage included more internal variation (niches) based on site differencies and physiological activities of the fungi. The greater variation reflect a real ecological heterogeneity of the trunks at this decay stage, or it may be due to a deficiency in the decay classification.

\section{Species composition}

Altogether 166 species of wood-inhabiting Basidiomycetes were recorded on fallen trunks of Picea abies and Pinus sylvestris; 120 species were found on spruce and 104 on pine. Of the 166 species, $13.3 \%$ (16) of the species on spruce and $21.6 \%$ (22) of those on pine were brown rotters. All the taxa, from both spruce and pine trunks, are summarized in Table 4, together with data on their preference for certain stages of trunk decay, and the number of observations. The number of species on trunks at different stages of decomposition increased from

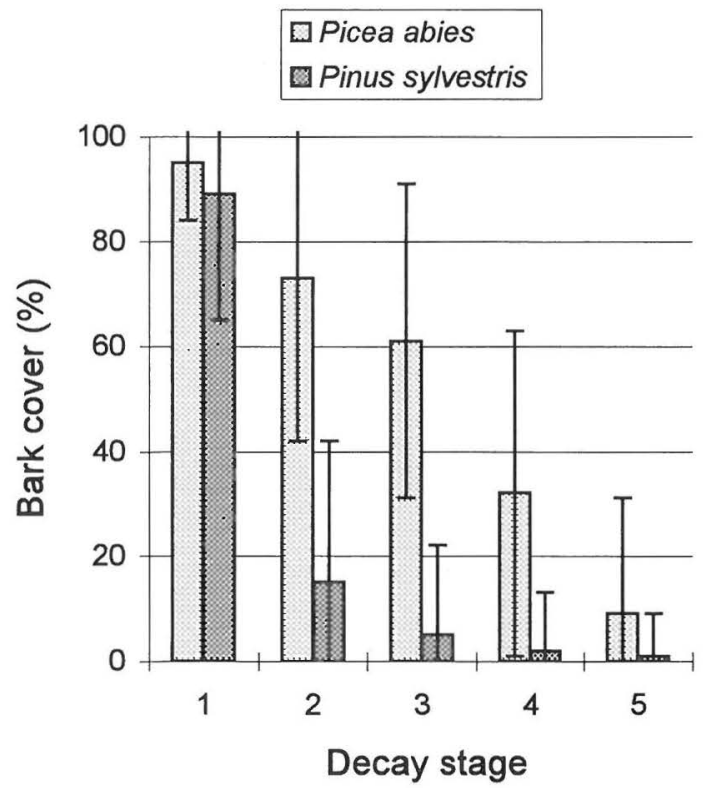

Fig. 2. Percentage of bark (meantone standard deviation) remaining on sample trunks of Picea abies subsp. obovata $(\mathrm{n}=320)$ and Pinus sylvestris $(\mathrm{n}=440)$ at different stages of decay.
32 species ( $19.2 \%$ of the total number of species) at decay stage 1 to 113 species $(68.1 \%)$ at decay stage 4 (Fig. 4). Of the total number of species, $37.4 \%$ (62 species) were found exclusively on spruce (Table 5) and $27.7 \%$ (46 species) exclusively on pine (Table $6)$.

Only a few species were recorded frequently on sample trunks, and the great majority were found less than ten times. On spruce, eight species $(6.7 \%$ of all the species on spruce) occurred on more than $10 \%$ of the trunks, and 70 species $(58.3 \%)$ were found on less than $1 \%$ (Table 7). On pine, only three species ( $2.9 \%$ of all the species on pine) were found on more than $10 \%$ of the trunks, and 62 species $(59.6 \%$ ) on less than $1 \%$ (Table 8 ). However, as shown below (Tables 14 and 19), each stage of decay harboured at least one clearly dominant species. The five most frequently occurring species on spruce trunks were Fomitopsis rosea, Phellinus nigrolimitatus, Trichaptum abietinum, Phlebia centrifuga and Amylocystis lapponica. On pine the five most frequently occurring species were Amyloporia xantha, Piloderma croceum, Antrodia albobrunnea, Stereum sanguinolentum and Skeletocutis lenis. Five out of the ten most frequently occurring species on spruce, and four on pine, were brown-rotters.

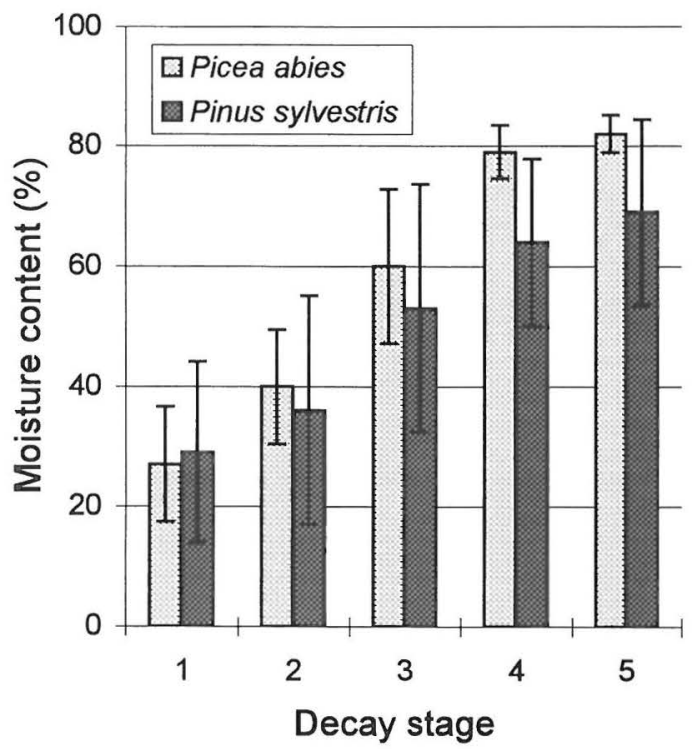

Fig. 3. Moisture content ( $\%$ of wet mass; meantone standard deviation) of wood samples from selected trunks of Picea abies subsp. obovata $(\mathrm{n}=50)$ and Pinus sylvestris $(n=50)$ at different stages of decay $(1-5)$. 
Table 4. List of Basidiomycetes found on fallen trunks of Pinus sylvestris $(\mathrm{n}=440)$ and Picea abies subsp. obovata $(n=320)$ in northeastern Finland. Species are arranged according to their preference for stage of trunk decay $(1-5, n=$ number of observations). Species that cause brown rot are marked with *. Species printed in bold face have been classified as threatened in Finland (unpublished revised list).

Species

$\begin{array}{ll}\text { Decay stage } & \mathrm{n} \\ \text { mean } & \text { range }\end{array}$

Amphinema byssoides (Fr.) J. Eriksson

Calocera viscosa (Pers.:Fr.) Fr.

Gelatoporia pannocincta (Romell) Niemelä

Panellus mitis (Pers.: Fr.) P. Singer

$P$. serotinus (Schrad.: Fr.) Kühner

Peniophora pini (Fr.) Boidin

Sistotrema brinkmannii (Bres.) J. Eriksson*

Exidia saccharina (Alb. \& Schwein.: Fr.) Fr.

Phlebiopsis gigantea (Fr.) Jülich

Peniophora septentrionalis Laurila

Stereum sanguinolentum (Alb. \& Schwein.: Fr.) Fr.

Hyphoderma setigerum (Fr.) Donk

Athelia fibulata M.P. Christ.

Cylindrobasidium laeve (Pers.: Fr.) Chamuris

Trichaptum fuscoviolaceum (Ehrenb.: Fr.) Ryvarden

Onnia leporina (Fr.) Jahn

Diplomitoporus crustulinus (Bres.) Dom.

Fibricium rude (P. Karsten) Jülich

Hyphodontia subalutacea (P. Karsten) J. Eriksson

Phlebia tremellosa (Schrad.: Fr.) Burds. \& Nakasone

Postia caesia (Schrad.: Fr.) P. Karsten*

Skeletocutis amorpha (Fr.) Kotl. \& Pouzar

S. lilacina A. David \& Jean Keller

Sphaerobasidium minutum (J. Eriksson) Oberw.

Peniophora pithya (Pers.) J. Eriksson

Leptoporus mollis (Pers.: Fr.) Quél.*

Climacocystis borealis (Fr.) Kotlaba \& Pouzar

Gloeophyllum sepiarium (Wulfen: Fr.) P. Karsten*

Phlebia centrifuga P. Karsten

Athelia cf. acrospora Jülich

Coniophora arida (Fr.) P. Karsten*

Gloeoporus taxicola (Pers.: Fr.) Gilb. \& Ryvarden

Hyphodontia hastata (Litsch.) J. Eriksson

Tomentella radiosa (P. Karsten) Rick

$\begin{array}{lll}1 & - & 1 \\ 1 & - & 1 \\ 1 & - & 1 \\ 1 & - & 7 \\ 1 & - & 1 \\ 1 & - & 1 \\ 1 & - & 1 \\ 1.2 & 1-3 & 20 \\ 1.2 & 1-2 & 12 \\ 1.2 & 1-2 & 5 \\ 1.2 & 1-3 & 82 \\ 1.3 & 1-2 & 18 \\ 1.5 & 1-2 & 2 \\ 1.5 & 1-2 & 2 \\ 1.5 & 1-3 & 44 \\ 1.9 & 1-4 & 23\end{array}$

Amyloporia xantha (Fr.: Fr.) Bondartsev \& Singer*

Antrodia primaeva Renvall \& Niemelä*

Fomitopsis pinicola (Sw.: Fr.) P. Karsten*

Gloeophyllum protractum (Fr.) Imazeki*

Sistotrema muscicola (Pers.) S. Lundell

Skeletocutis odora (Sacc.) Ginns

Trichaptum abietinum (Pers.: Fr.) Ryvarden

Phellinus chrysoloma (Fr.) Donk

1 1 7 
Table 4. Contd.

\begin{tabular}{|c|c|c|c|}
\hline Species & mean & rangee & $\mathrm{n}$ \\
\hline Amylocystis lapponica (Romell) Singer* & 2.9 & $2-4$ & 43 \\
\hline Antrodia infirma Renvall \& Niemelä* & 3 & - & 4 \\
\hline Antrodiella citrinella Niemelä \& Ryvarden & 3 & - & 1 \\
\hline Columnocystis abietina (Fr.) Pouzar* & 3 & $1-4$ & 29 \\
\hline Coniophora olivacea (Fr.: Fr.) P. Karsten* & 3 & $1-4$ & 36 \\
\hline Dichomitus squalens (P. Karsten) D.A. Reid & 3 & - & 1 \\
\hline Gelatoporia subvermispora (Pilát) Niemelä & 3 & - & 1 \\
\hline Hyphoderma cf. sibiricum (Parmasto) J. Eriksson \& Strid & 3 & - & 1 \\
\hline Lentaria epichnoa (Fr.) Corner & 3 & - & 1 \\
\hline Lentinellus castoreus (Fr.) Konrad \& Maublanc & 3 & - & 1 \\
\hline Leptosporomyces fuscoatrus (Burt) Hjortstam & 3 & - & 1 \\
\hline Omphalina oniscus (Fr.: Fr.) Quél. & 3 & - & 1 \\
\hline Phanerochaete sordida (P. Karsten) J. Eriksson \& Ryvarden & 3 & - & 1 \\
\hline Phellinus viticola (Schwein. ex Fr.) Donk & 3 & $2-5$ & 39 \\
\hline Phlebia cretacea (Bourdot \& Galzin) J. Eriksson \& Hjortstam & 3 & - & 1 \\
\hline Phlebiella borealis Larsson \& Hjortstam & 3 & - & 1 \\
\hline Postia lateritia Renvall\% & 3 & $2-4$ & 24 \\
\hline Pseudotomentella $\mathrm{sp.} 1$ & 3 & - & 1 \\
\hline Tomentella ellisii (Saccardo) Jülich \& Stalpers & 3 & - & 1 \\
\hline Vararia racemosa (Burt) Rog. \& Jacks. ssp. lapponica Hallenb. & 3 & - & 1 \\
\hline Amylostereum chailletii (Fr.) Boidin & 3.1 & $1-4$ & 11 \\
\hline Trichaptum laricinum (P. Karsten) Ryvarden & 3.1 & $2-5$ & 14 \\
\hline Aleurodiscus lividocoeruleus (P. Karsten) Lemke & 3.2 & $2-4$ & 5 \\
\hline Amyloporia crassa (P. Karsten) Bondartsev \& Singer* & 3.3 & $3-4$ & 4 \\
\hline Athelia decipiens (v. Höhn. \& Litsch.) J. Eriksson & 3.3 & $3-4$ & 4 \\
\hline Ischnoderma benzoinum (Wahlenb.: Fr.) P. Karsten & 3.3 & $2 ; 4$ & 3 \\
\hline Hyphodontia alutaria (Burt) J. Eriksson & 3.3 & $2-3 ; 5$ & 4 \\
\hline Postia hibernica (Berk. \& Broome) Jülich* & 3.3 & $2-5$ & 27 \\
\hline Tubulicrinis borealis J. Eriksson & 3.3 & $2 ; 4$ & 6 \\
\hline Antrodia albobrunnea (Romell) Ryvarden* & 3.4 & $2-5$ & 51 \\
\hline A. serialis (Fr.) Donk: & 3.4 & $2-4$ & 27 \\
\hline Antrodiella parasitica Vampola & 3.4 & $3-4$ & 17 \\
\hline Botryobasidium botryosum (Bres.) J. Eriksson & 3.4 & $2-5$ & 34 \\
\hline Mycena epipterygia (Scop.: Fr.) S.F. Gray & 3.4 & $2-4$ & 9 \\
\hline Phlebia cornea (Bourdot \& Galzin) J. Eriksson & 3.4 & $2-4$ & 7 \\
\hline Amylocorticium cebennense (Bourdot) Pouzar* & 3.5 & $3 ; 5$ & 2 \\
\hline Athelia epiphylla Pers. & 3.5 & $3-4$ & 2 \\
\hline Hyphodontia pallidula (Bres.) J. Eriksson & 3.5 & $3-4$ & 4 \\
\hline Laurilia sulcata (Burt) Pouzar & 3.5 & $2-5$ & 23 \\
\hline Oligoporus sericeomollis (Romell) Pouzar" & 3.5 & $2-4$ & 24 \\
\hline Phanerochaete velutina (Fr.) P. Karsten & 3.5 & $3-4$ & 2 \\
\hline Physodontia lundellii Ryvarden \& Solheim & 3.5 & $3-4$ & 2 \\
\hline Sistotremastrum suecicum (v. Höhn. \& Litsch.) J. Eriksson & 3.5 & $2-5$ & 11 \\
\hline Tubulicrinis subulatus (Bourdot \& Galzin) Donk & 3.5 & $3-4$ & 2 \\
\hline Ceraceomyces sublaevis (Bres.) Jülich & 3.6 & $2-4$ & 10 \\
\hline C. borealis (Romell) J. Eriksson \& Ryvarden & 3.7 & $2-5$ & 9 \\
\hline Hyphoderma praetermissum (P. Karsten) J. Eriksson \& Strid & 3.7 & $2-5$ & 22 \\
\hline Hypochnicium albostramineum (Bres.) Hallenb. & 3.7 & $3-4$ & 6 \\
\hline Piloderma olivaceum (Parmasto) Hjortstam & 3.7 & $3-4$ & 6 \\
\hline Pseudotomentella tristis (P. Karsten) M.J. Larsen & 3.7 & $3 ; 5$ & 3 \\
\hline Skeletocutis kuehneri A. David & 3.7 & $3-4$ & 3 \\
\hline S. subincarnata (Peck) Jean Keller & 3.7 & $3-4$ & 3 \\
\hline
\end{tabular}


Table 4. Contd.

\begin{tabular}{|c|c|c|c|}
\hline Species & mean & range & $\mathrm{n}$ \\
\hline Trechispora farinacea (Pers.: Fr.) Liberta & 3.7 & $2-5$ & 27 \\
\hline Tubulicrinis medius (Bourdot \& Galzin) Oberw. & 3.7 & $3-4$ & 7 \\
\hline Dichostereum granulosum (Fr.) Boidin \& Lanquetin & 3.8 & $3-4$ & 5 \\
\hline Globulicium hiemale (Laurila) Hjortstam & 3.8 & $3-5$ & 11 \\
\hline Leucogyrophana romellii Ginns* & 3.8 & $2-5$ & 24 \\
\hline Mucronella calva (Alb. \& Schwein. ex Schwein.) Fr. & 3.8 & $3-4$ & 5 \\
\hline Phanerochaete laevis (Fr.) J. Eriksson \& Ryvarden & 3.8 & $3-5$ & 4 \\
\hline P. sanguinea (Fr.) Pouzar & 3.8 & $3-5$ & 11 \\
\hline Phellinus nigrolimitatus (Romell) Bourdot \& Galzin & 3.8 & $2-5$ & 64 \\
\hline Piloderma croceum (P. Karsten) Jülich & 3.8 & $2-5$ & 73 \\
\hline Resinicium furfuraceum (Bres.) Parmasto & 3.8 & $2-5$ & 19 \\
\hline Tomentellopsis echinospora (Ellis) Hjortstam & 3.8 & $3-4$ & 5 \\
\hline Asterodon ferruginosus (Pat.) Parmasto & 3.9 & $3-5$ & 10 \\
\hline Hyphodontia aspera (Fr.) J. Eriksson & 3.9 & $3-5$ & 7 \\
\hline H. breviseta (P. Karsten) J. Eriksson & 3.9 & $3-5$ & 23 \\
\hline Hyphoderma argillaceum (Bres.) Donk & 3.9 & $2 ; 4-5$ & 9 \\
\hline Phlebiella vaga (Fr.) P. Karsten & 3.9 & $2-5$ & 41 \\
\hline Tricholomopsis decora (Fr.) Singer & 3.9 & $3-4$ & 7 \\
\hline Tubulicrinis calothrix (Pat.) Donk & 3.9 & $2-4$ & 15 \\
\hline Botryobasidium candicans J. Eriksson & 4 & - & 1 \\
\hline B. subcoronatum (v. Höhn. \& Litsch.) Donk & 4 & $2-5$ & 38 \\
\hline Byssocorticium terrestre (DC.: Fr.) Bondartsev \& Singer & 4 & $3 ; 5$ & 2 \\
\hline Ceraceomerulius serpens (Fr.) J. Eriksson \& Ryvarden & 4 & - & 1 \\
\hline Crepidotus subsphaerosporus (Lange) Kühner \& Romagn. & 4 & - & 1 \\
\hline Fibulomyces mutabilis (Bres.) Jülich & 4 & - & 1 \\
\hline F. septentrionalis (J. Eriksson) Jülich & 4 & - & 1 \\
\hline Conferticium ochraceum (Fr.: Fr.) Hallenb. & 4 & - & 1 \\
\hline Gymnopilus penetrans (Fr.) Murrill & 4 & - & 1 \\
\hline Hymenochaete fuliginosa (Pers.) Bres. & 4 & $3 ; 5$ & 2 \\
\hline Hyphoderma cremeoalbum (V. Höhn. \& Litsch.) Jülich & 4 & - & 2 \\
\hline H. pallidum (Bres.) Donk & 4 & - & 1 \\
\hline Hyphodontia alutacea (Fr.) J. Eriksson & 4 & - & 2 \\
\hline H. cineracea (Bourdot \& Galzin) J. Eriksson \& Hjortstam & 4 & - & 1 \\
\hline Kavinia alboviridis (Morgan) Gilb. \& Budington & 4 & - & 1 \\
\hline Leucogyrophana mollusca (Fr.) Pouzar* & 4 & - & 1 \\
\hline Mucronella bresadolae (Quél.) Corner & 4 & - & 1 \\
\hline Odonticium romellii (S. Lundell) Parmasto & 4 & - & 5 \\
\hline Phlebia segregata (Bourdot \& Galzin) Parmasto & 4 & - & 4 \\
\hline Phlebiella pseudotsugae (Burt) Larsson \& Hjortstam & 4 & $3-5$ & 6 \\
\hline Piloporia sajanensis (Parmasto) Niemelä & 4 & - & 1 \\
\hline Postia placenta (Fr.) M.J. Larsen \& Lombard** & 4 & - & 1 \\
\hline Protodontia piceicola (Kühner ex Bourdot) Martin & 4 & - & 1 \\
\hline Pseudotomentella nigra (Höhn. \& Litsch.) Svrček & 4 & $3-5$ & 2 \\
\hline Scytinostromella nannfeldtii (J. Eriksson) Freeman \& Petersen & 4 & - & 1 \\
\hline Sistotrema sernanderi (Litsch.) Donk & 4 & - & 1 \\
\hline Skeletocutis jelicii Tortic \& A. David & 4 & - & 1 \\
\hline Skeletocutis sp. 1 & 4 & - & 1 \\
\hline Serpula himantioides (Fr.: Fr.) P. Karsten** & 4 & $3-5$ & 4 \\
\hline Tomentella bryophila (Pers.: Fr.) M.J. Larsen & 4 & - & 1 \\
\hline T. stuposa (Link) Stalpers & 4 & - & 1 \\
\hline T. sublilacina (Ellis \& Holway) Wakefield & 4 & - & 1 \\
\hline Trechispora subsphaerospora (Litsch.) Liberta & 4 & - & 1 \\
\hline Tubulicrinis accedens (Bourdot \& Galzin) Donk & 4 & - & 1 \\
\hline
\end{tabular}


Table 4. Contd.

\begin{tabular}{|c|c|c|c|}
\hline Species & mean & range & $\mathrm{n}$ \\
\hline T. chaetophorus (v. Höhn.) Donk & 4 & - & 2 \\
\hline T. globisporus Larsson \& Hjortstam & 4 & - & 3 \\
\hline T. gracillimus (Rog. \& Jacks.) G.H. Cunn. & 4 & - & 1 \\
\hline Skeletocutis lenis (P. Karsten) Niemelä & 4.2 & $2-5$ & 36 \\
\hline S. stellae (Pilát) Jean Keller & 4.2 & $3-5$ & 7 \\
\hline Piloderma byssinum (P. Karsten) Jülich & 4.2 & $1-2 ; 4-5$ & 11 \\
\hline Gloiothele citrina (Pers.) Ginns \& Freeman & 4.2 & $3-5$ & 5 \\
\hline Pseudotomentella mucidula (P. Karsten) Svrček & 4.3 & $4-5$ & 3 \\
\hline Tylospora fibrillosa (Burt) Donk & 4.4 & $3-5$ & 12 \\
\hline Leptosporomyces galzinii (Bourdot) Jülich & 4.5 & $4-5$ & 2 \\
\hline Tubulicrinis effugiens (Bourdot \& Galzin) Oberw. & 4.5 & $4-5$ & 2 \\
\hline Botryobasidium angustisporum Boidin & 5 & - & 2 \\
\hline Phlebiella subflavidogrisea (Litsch.) Oberw. & 5 & - & 1 \\
\hline
\end{tabular}

Comparison of the mycoflora in the different forest site types showed that, although the basic composition of the fungi on pine or spruce remained the same, each site type also exhibited characteristics of its own. On spruce, for example, Leptoporus mollis, Onnia leporina and Stereum sanguinolentum occurred almost exclusively in mesic forests of the Hylocomium-Myrtillus type, while they were virtually lacking in brookside forests (Tables 9 and 10). Laurilia sulcata and Phellinus chrysoloma were more frequent in brookside forests, and Amylostereum chailletii, Climacocystis borealis, Hyphoderma argillaceum, Mycena epipterygia and Skeletocutis stellae were found almost exclusively in brookside forests.

The variation in species composition of fungi between different site types was less marked on pine than on spruce (Tables 11 and 12). However, pine-inhabiting Chaetodermella luna, Leucogyrophana romellii, Postia hibernica and Sistotrema muscicola occurred more frequently in very dry areas of the Uliginosum-VacciniumEmpetrum site type than in more mesic localities. Antrodia primaeva, Junghuhnia luteoalba, Postia lateritia and the rarities Amyloporia crassa and Antrodia infirma, in turn, favoured the somewhat mesic forests of the Empetrum-Myrtillus type. Moreover, pine trunks in some of the exceptionally mesic and fertile sites harboured a few species that almost exclusively grow on spruce in northern Europe. Accordingly, all the records of Amylocystis lapponica, Columnocystis abietina, Fomitopsis rosea, Phellinus ferrugineofuscus, $P$. nigro- limitatus and Skeletocutis odora on pine derive from continuously moist habitats, often depressions, of the Empetrum-Myrtillus type.

Charred pine trunks. The mycoflora of pine trunks heavily damaged by fire exhibited some unique features. Altogether 29 species were found on charred wood. Piloderma croceum was the most frequent fungus and was present on $54.9 \%$ of the charred trunks, though mostly only as rhizomorphs. It was especially frequent on strongly decayed charred trunks (D.S. 4). Also Antrodia primaeva, Leucogyrophana romellii, Ceraceomyces borealis and Sistotrema muscicola favoured charred wood (Table 13), whereas Amyloporia xantha, Postia hibernica, $P$. lateritia and many other regular members of the mycoflora on pine trunks were almost absent from such trunks.

Sequence, diversity and organization of fungi on spruce trunks

Sequence. Compositions of the fungal communities on individual spruce trunks varied widely with the stage of decomposition, which means with the softness of the wood and the amount of bark on the trunk. Each fungus had a specific preference for trunks at a certain stage of decay (Table 14). Freshly fallen, still corticated, undecayed trunks harboured only a few species that were also found on extensively decayed, decorticated trunks at late stages of decomposition (Table 15). On the other hand, only $13.6 \%$ of the species that inhabited 
Table 5. List of Basidiomycetes found exclusively on Picea abies subsp. obovata. Brown rot species are marked with *.

\begin{tabular}{ll}
\hline Amphinema byssoides & Onnia leporina \\
Amylostereum chailletii & Panellus serotinus \\
Antrodia serialis & Peniophora pithya \\
Antrodiella citrinella & P. septentrionalis \\
Athelia epiphylla & Phanerochaete laevis \\
A. fibulata & P. sordida \\
Botryobasidium angustisporum & P. velutina \\
Calocera viscosa & Phellinus chrysoloma \\
Ceraceomerulius serpens & Phlebia centrifuga \\
Climacocystis borealis & P. tremellosa \\
Conferticium ochraceum & Piloporia sajanensis \\
Crepidotus subsphaerosporus & Postia caesia \\
Cylindrobasidium laeve & P. placenta \\
Dichomitus squalens & Protodontia piceicola \\
Dichostereum granulosum & Pseudotomentella mucidula \\
Diplomitoporus crustulinus & P. nigra \\
Fibricium rude & Scytinostromella nannfeldtii \\
Fibulomyces septentrionalis & Sistotrema brinkmannii \\
Gelatoporia pannocincta & S. sernanderi \\
G. subvermispora & Skeletocutis lilacina \\
Gloiothele citrina & Skeletocutis sp. 1 \\
Hymenochaete fuliginosa & Sphaerobasidium minutum \\
Hyphoderma cremeoalbum & Tomentella bryophila \\
Hyphodontia cineracea & T. ellisii \\
Kavinia alboviridis & T. stuposa \\
Laurilia sulcata & T. sublilacina \\
Lentaria epichnoa & Trechispora subsphaerospora \\
Lentinellus castoreus & Tubulicrinis accedens \\
Leptoporus mollis $*$ & T. subulatus \\
Mycena epipterygia & Tylospora fibrillosa \\
Omphalina oniscus & Vararia racemosa ssp. lapponica \\
& \\
&
\end{tabular}

Table 6. List of Basidiomycetes found exclusively on Pinus sylvestris. Brown rot species are marked with *.

Amyloporia crassa*

A. xantha*

Antrodia albobrunnea*

A. infirma*

A. primaeva*

A. sinuosa*

Athelia cf. acrospora

A. decipiens

Botryobasidium candicans

Chaetodermella luna*

Fibulomyces mutabilis

Gloeophyllum protractum*

Gymnopilus penetrans

Hyphoderma pallidum

H. setigerum

H. cf. sibiricum

Hyphodontia subalutacea
Junghuhnia luteoalba

Leptosporomyces fuscoatrus

L. galzinii

Leucogyrophana mollusca*

Mucronella bresadolae

Odonticium romellii

Peniophora pini

Piloderma olivaceum

Phlebia cornea

P. cretacea

P. segregata

Phlebiella borealis

P. subflavidogrisea

Physodontia lundellii

Postia hibernica :

P. lateritia*

Pseudotomentella sp. 1 
Table 6. Contd.

Sistotrema muscicola

Sistotremastrum suecicum

Skeletocutis amorpha

S. jelicii

S. lenis

S. subincarnata
Tomentella radiosa

Tubulicrinis chaetophorus

T. effugiens

T. globisporus

T. gracillimus

T. medius sample trunks at decay stages 4 and 5 could be found on more or less intact trunks (D.S. 1).

Typical pioneers on freshly fallen spruce trunks (D.S. 1) were Stereum sanguinolentum and Exidia saccharina. In addition, Peniophora septentrionalis was found on thin (base diameter $20-30 \mathrm{~cm}$ ) trunks that were evidently broken by heavy snow during winter but remained attached
Table 7. The frequency of occurrence of wood-inhabiting Basidiomycetes on fallen trunks of Picea abies $(n=320)$. Brown rot species are marked with $*$ freq $=$ frequency (in $\%) ; n=$ number of observations. Species recorded less than ten times have been omitted.

\begin{tabular}{|c|c|c|}
\hline Species & freq & $\mathrm{n}$ \\
\hline Fomitopsis rosea & 24.7 & 79 \\
\hline Phellinus nigrolimitatus & 17.5 & 56 \\
\hline Trichaptum abietinum & 17.1 & 55 \\
\hline Phlebia centrifuga & 14.4 & 46 \\
\hline Amylocystis lapponica* & 13.1 & 42 \\
\hline Stereum sanguinolentum & 12.8 & 41 \\
\hline Fomitopsis pinicola* & 11.9 & 38 \\
\hline Gloeophyllum sepiarium & 10.3 & 33 \\
\hline Phellinus chrysoloma & 9.4 & 30 \\
\hline Coniophora olivacea & 9.1 & 29 \\
\hline Phellinus ferrugineofuscus & 9.1 & 29 \\
\hline Columnocystis abietina* & 8.8 & 28 \\
\hline Antrodia serialis & 8.4 & 27 \\
\hline Laurilia sulcata & 7.2 & 23 \\
\hline Onnia leporina & 7.2 & 23 \\
\hline Skeletocutis odora & 6.9 & 22 \\
\hline Piloderma croceum & 6.3 & 20 \\
\hline Botryobasidium subcoronatum & 4.7 & 15 \\
\hline Hyphodontia breviseta & 4.7 & 15 \\
\hline Phlebiella vaga & 4.4 & 14 \\
\hline Tubulicrinis calothrix & 4.1 & 13 \\
\hline Antrodiella parasitica & 3.8 & 12 \\
\hline Gloeoporus taxicola & 3.8 & 12 \\
\hline Tylospora fibrillosa & 3.8 & 12 \\
\hline Amylostereum chailletii & 3.4 & 11 \\
\hline Phellinus viticola & 3.4 & 11 \\
\hline Trichaptum fuscoviolaceum & 3.1 & 10 \\
\hline
\end{tabular}

Table 8. The frequency of occurrence (in \%) of woodinhabiting Basidiomycetes on fallen trunks of Pinus sylvestris $(\mathrm{n}=440)$. Brown rot species are marked with *; freq $=$ frequency (in $\%$ ); $\mathrm{n}=$ number of observations. Species recorded less than ten times have been omitted.

\begin{tabular}{|c|c|c|}
\hline Species & freq & $\mathrm{n}$ \\
\hline Amyloporia xantha* & 15.9 & 70 \\
\hline Piloderma croceum & 12.7 & 56 \\
\hline Antrodia albobrunnea* & 11.6 & 51 \\
\hline Stereum sanguinolentum & 9.3 & 41 \\
\hline Skeletocutis lenis & 8.2 & 36 \\
\hline Trichaptum fuscoviolaceum & 7.7 & 34 \\
\hline Chaetodermella luna* & 7.1 & 31 \\
\hline Botryobasidium botryosum & 6.6 & 29 \\
\hline Phellinus viticola & 6.4 & 28 \\
\hline Phlebiella vaga & 6.1 & 27 \\
\hline Postia hibernica* & 6.1 & 27 \\
\hline P. lateritia* & 5.5 & 24 \\
\hline Trechispora farinacea & 5.5 & 24 \\
\hline Antrodia primaeva* & 5.2 & 23 \\
\hline Botryobasidium subcoronatum & 5.2 & 23 \\
\hline Leucogyrophana romellii* & 5.2 & 23 \\
\hline Oligoporus sericeomollis & 4.3 & 19 \\
\hline Resinicium furfuraceum & 4.1 & 18 \\
\hline Hyphoderma setigerum & 4.1 & 18 \\
\hline Antrodia sinuosa* & 3.9 & 17 \\
\hline H. praetermissum & 3.4 & 15 \\
\hline Exidia saccharina & 3.0 & 13 \\
\hline Phlebiopsis gigantea & 2.5 & 11 \\
\hline Sistotremastrum suecicum & 2.5 & 11 \\
\hline Globulicium hiemale & 2.3 & 10 \\
\hline Trichaptum abietinum & 2.3 & 10 \\
\hline
\end{tabular}


Table 9. The frequency of occurrence of the 20 commonest Basidiomycetes on fallen trunks of Picea abies $(n=220)$ in mesic spruce forests (HMT site type). Brown rot species are marked with $*$; freq $=$ frequency $($ in $\%) ; n=$ number of observations.

\begin{tabular}{|c|c|c|}
\hline Species & freq & $\mathrm{n}$ \\
\hline Fomitopsis roseat & 27.3 & 60 \\
\hline Phellinus nigrolimitatus & 18.6 & 41 \\
\hline Stereum sanguinolentum & 16.4 & 36 \\
\hline Trichaptum abietinum & 15.9 & 35 \\
\hline Amylocystis lapponica* & 13.6 & 30 \\
\hline Phlebia centrifuga & 12.3 & 27 \\
\hline Gloeophyllum sepiarium* & 11.8 & 26 \\
\hline Fomitopsis pinicolat & 10.5 & 23 \\
\hline Phellinus ferrugineofuscus & 9.6 & 21 \\
\hline Onnia leporina & 9.1 & 20 \\
\hline Antrodia serialis* & 8.2 & 18 \\
\hline Columnocystis abietina* & 8.2 & 18 \\
\hline Phellinus chrysoloma & 7.7 & 17 \\
\hline Piloderma croceum & 7.3 & 16 \\
\hline Skeletocutis odora & 6.8 & 15 \\
\hline Coniophora olivacea* & 5.9 & 13 \\
\hline Laurilia sulcata & 5.0 & 11 \\
\hline Antrodiella parasitica & 4.6 & 10 \\
\hline Phlebiella vaga & 4.6 & 10 \\
\hline Gloeoporus taxicola & 4.1 & 9 \\
\hline Leptoporus mollis* & 4.1 & 9 \\
\hline Trichaptum fuscoviolaceum & 4.1 & 9 \\
\hline Tubulicrinis calothrix & 4.1 & 9 \\
\hline
\end{tabular}

to the stump, thus constituting special microclimatical conditions. Depending on the successional pathway, the trunks at decay stage 2 were often occupied by Fomitopsis pinicola, Onnia leporina, Phellinus chrysoloma or the co-occurring Fomitopsis rosea and Amylocystis lapponica. Fomitopsis rosea was recorded on $47 \%$ and Amylocystis lapponica on $23 \%$ of the trunks. Very often these species co-occurred with Phlebia centrifuga. Trichaptum abietinum was abundant on trunks which were primarily decayed by Onnia leporina, Phellinus chrysoloma or Fomitopsis pinicola. The predominant fungi on trunks at decay stage 3 were Fomitopsis rosea (on $45.5 \%$ of the trunks), Amylocystis lapponica (22\%), Phellinus chrysoloma, P. ferrugineofuscus and Skeletocutis odora. The trunk crowns were almost always decorated with Antrodia serialis, Columnocystis abietina or Gloeophyllum sepiarium. The extensively decayed spruce trunks (D.S. 4) maintained a high species diversity, but only a
Table 10. The frequency of occurrence of the 20 commonest Basidiomycetes on fallen trunks of Picea abies $(\mathrm{n}=100)$ in brookside forests (GDMT site type). Brown rot species are marked with $*$; freq $=$ frequency $($ in $\%$ ); $\mathrm{n}=$ number of observations.

\begin{tabular}{|c|c|c|}
\hline Species & freq & $\mathrm{n}$ \\
\hline Fomitopsis rosea* & 24 & 24 \\
\hline Phlebia centrifuga & 19 & 19 \\
\hline Trichaptum abietinum & 19 & 19 \\
\hline Coniophora olivacea* & 16 & 16 \\
\hline Fomitopsis pinicola & 15 & 15 \\
\hline Phellinus nigrolimitatus & 15 & 15 \\
\hline Amylocystis lapponica* & 12 & 12 \\
\hline Laurilia sulcata & 12 & 12 \\
\hline Phellinus chrysoloma & 11 & 11 \\
\hline Columnocystis abietina* & 10 & 10 \\
\hline Antrodia serialis* & 9 & 9 \\
\hline Amylostereum chailletii & 8 & 8 \\
\hline Phellinus ferrugineofuscus & 8 & 8 \\
\hline Botryobasidium subcoronatum & 7 & 7 \\
\hline Hyphoderma argillaceum & 7 & 7 \\
\hline Hyphodontia breviseta & 7 & 7 \\
\hline Mycena epipterygia & 7 & 7 \\
\hline Climacocystis borealis & 6 & 6 \\
\hline Gloeophyllum sepiarium* & 6 & 6 \\
\hline Skeletocutis odora & 6 & 6 \\
\hline
\end{tabular}

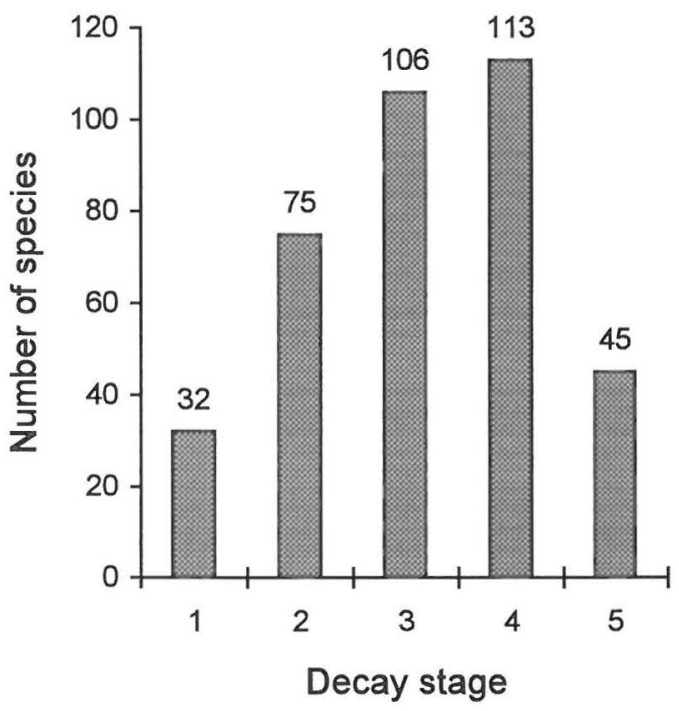

Fig. 4. The total number of Basidiomycete species on fallen trunks of Picea abies subsp. obovata $(\mathrm{n}=320)$ and Pinus sylvestris $(\mathrm{n}=440)$ at different stages of decay $(1-5)$. Number of sample trunks at each stage of decay: D.S. $1-$ 4, 170 trunks at each stage; D.S. 5, 80 trunks. 
Table 11. The frequency of occurrence of the 15 commonest species of Basidiomycetes on fallen trunks of Pinus sylvestris $(\mathrm{n}=220)$ in dry pine forests (UVET site type). Brown rot species are marked with *; freq $=$ frequency (in $\%$ ); $\mathrm{n}=$ number of observations.

\begin{tabular}{lrr}
\hline Species & freq & $\mathrm{n}$ \\
\hline Amyloporia xantha* & 17.7 & 39 \\
Piloderma croceum $^{*}$ Antrodia albobrunnea* & 16.8 & 37 \\
Stereum sanguinolentum & 13.6 & 30 \\
Leucogyrophana romellit* $_{\text {Phlebiella vaga }}^{*}$ & 9.6 & 21 \\
Postia hibernica* & 8.2 & 18 \\
Skeletocutis lenis & 8.2 & 18 \\
Hyphoderma setigerum & 7.7 & 17 \\
Trichaptum fuscoviolaceum & 7.3 & 16 \\
Chaetodermella luna* & 6.8 & 15 \\
Trechispora farinacea & 6.8 & 15 \\
Phellinus viticola & 5.9 & 13 \\
Phlebiopsis gigantea & 5.9 & 13 \\
Resinicium furfuraceum & 4.6 & 10 \\
& 4.6 & 10 \\
\hline
\end{tabular}

very few fungi were recorded repeatedly on these trunks. Phellinus nigrolimitatus, which often was recorded at decay stage 3 as well, was the dominant decayer of trunks at decay stage 4 . It was present on $50 \%$ of trunks at stage 4 . In brookside forests also Laurilia sulcata, Hyphoderma argillaceum, Hyphodontia breviseta and Skeletocutis stellae belong to the characteristic mycoflora of decay stage 4. Almost completely decomposed trunks (D.S. 5) were characterized by Piloderma croceum, Tylospora fibrillosa and many other corticiaceous fungi.

The mycoflora of decorticated spruce trunks was mostly different from that on the still corticated trunks, and each species preferred either corticated or naked wood (Table 16). The fruiting of Peniophora septentrionalis, Climacocystis borealis, Stereum sanguinolentum and Trichaptum spp. seemed to depend on the presence of bark on the sample trunks, while many other fungi, e.g., Columnocystis abietina and many species of Tubulicrinis, emerged almost exclusively on naked wood.

Diversity. The number of species on a single spruce trunk ranged from 0 (D.S. 1 and 5) to 14 (D.S. 3) (Fig. 5). The mean species number per spruce trunk was 3.2 . If the almost completely
Table 12. The frequency of occurrence of the 15 commonest species of Basidiomycetes on fallen trunks of Pinus sylvestris $(\mathrm{n}=220)$ in somewhat mesic pine forests (EMT site type). Brown rot species are marked with *; freq $=$ frequency $($ in $\%) ; n=$ number of observations.

\begin{tabular}{lrr}
\hline Species & freq & $\mathrm{n}$ \\
\hline Amyloporia xantha* & 14.1 & 31 \\
Antrodia albobrunnea* & 9.6 & 21 \\
Botryobasidium botryosum & 9.1 & 20 \\
Skeletocutis lenis & 9.1 & 20 \\
Stereum sanguinolentum & 9.1 & 20 \\
Antrodia primaeva* & 8.6 & 19 \\
Phellinus viticola & 8.2 & 18 \\
Piloderma croceum & 8.2 & 18 \\
Postia lateritia* & 7.3 & 16 \\
Botryobasidium subcoronatum & 6.4 & 14 \\
Trichaptum fuscoviolaceum & 5.9 & 13 \\
Hyphoderma praetermissum & 4.6 & 10 \\
Oligoporus sericeomollis* & 4.6 & 10 \\
Postia hibernica* & 4.6 & 10 \\
Phlebiella vaga $^{*}$ & 4.1 & 9 \\
\hline
\end{tabular}

decayed trunks (D.S. 5) are excluded, the number of species increased with the stage of decay (Figs. 5 and 6). The trunks at decay stage 1 harboured 27 species while altogether 81 species were recorded on trunks at decay stage 4 . The

Table 13. Basidiomycetes found on strongly charred trunks of Pinus sylvestris $(\mathrm{n}=51)$. Brown rot species are marked with *. Species found only once are omitted; $n=$ number observations.

\begin{tabular}{lc}
\hline Species & $\mathrm{n}$ \\
\hline Piloderma croceum & 28 \\
Antrodia primaeva* & 8 \\
Botryobasidium botryosum & 7 \\
Leucogyrophana romellit* $^{*}$ & 7 \\
Antrodia albobrunnea* & 6 \\
Phlebiella vaga & 6 \\
Ceraceomyces borealis & 5 \\
Amyloporia xantha* & 4 \\
Sistotrema muscicola & 4 \\
Trechispora farinacea & 4 \\
Botryobasidium subcoronatum & 3 \\
Athelia cf. acrospora & 2 \\
Gloeophyllum protractum* & 2 \\
Hyphoderma praetermissum & 2 \\
Odonticium romellii & 2 \\
Oligoporus sericeomollis* & 2 \\
\hline
\end{tabular}


Table 14. List of Basidiomycetes found on fallen trunks of Picea abies subsp. obovata $(\mathrm{n}=320)$ in northeastern Finland. Species are arranged according to their preference for stage of trunk decay ( $1-5 ; \mathrm{n}=$ number of observations). Species that cause brown rot are marked with *.

\begin{tabular}{|c|c|c|c|c|c|c|c|}
\hline \multirow[t]{2}{*}{ Species } & \multicolumn{7}{|c|}{ Decay stage } \\
\hline & mean & 1 & 2 & 3 & 4 & 5 & $\mathrm{n}$ \\
\hline Amphinema byssoides & 1 & 100 & - & - & - & - & 1 \\
\hline Calocera viscosa & 1 & 100 & - & - & - & - & 1 \\
\hline Gelatoporia pannocincta & 1 & 100 & - & - & - & - & 1 \\
\hline Panellus mitis & 1 & 100 & - & - & - & - & 3 \\
\hline$P$. serotinus & 1 & 100 & - & - & - & - & 1 \\
\hline Phlebiopsis gigantea & 1 & 100 & - & - & - & - & 1 \\
\hline Sistotrema brinkmannii* & 1 & 100 & - & - & - & - & 1 \\
\hline Exidia saccharina & 1.1 & 86 & 14 & - & - & - & 7 \\
\hline Stereum sanguinolentum & 1.2 & 85 & 15 & - & - & - & 41 \\
\hline Peniophora septentrionalis & 1.2 & 80 & 20 & - & - & - & 5 \\
\hline Athelia fibulata & 1.5 & 50 & 50 & - & - & - & 2 \\
\hline Cylindrobasidium laeve & 1.5 & 50 & 50 & - & - & - & 2 \\
\hline Onnia leporina & 1.9 & 39 & 39 & 17 & 4 & - & 23 \\
\hline Trichaptum fuscoviolaceum & 1.9 & 40 & 30 & 30 & - & - & 10 \\
\hline Diplomitoporus crustulinus & 2 & - & 100 & - & - & - & 1 \\
\hline Fibricium rude & 2 & - & 100 & - & - & - & 1 \\
\hline Phlebia tremellosa & 2 & - & 100 & - & - & - & 1 \\
\hline Postia caesia* & 2 & - & 100 & - & - & - & 2 \\
\hline Skeletocutis lilacina & 2 & - & 100 & - & - & - & 1 \\
\hline Sphaerobasidium minutum & 2 & - & 100 & - & - & - & 1 \\
\hline Peniophora pithya & 2.2 & - & 83 & 17 & - & - & 6 \\
\hline Leptoporus mollis* & 2.3 & 11 & 44.5 & 44.5 & - & - & 9 \\
\hline Gloeophyllum sepiarium* & 2.4 & 19 & 28 & 50 & 3 & - & 33 \\
\hline Phlebia centrifuga & 2.4 & 15 & 37 & 39 & 9 & - & 46 \\
\hline Climacocystis borealis & 2.4 & - & 57 & 43 & - & - & 7 \\
\hline Fomitopsis pinicola* & 2.6 & 8 & 45 & 26 & 21 & - & 38 \\
\hline Trichaptum abietinum & 2.6 & 18 & 29 & 29 & 20 & 4 & 55 \\
\hline Skeletocutis odora & 2.6 & 14 & 23 & 50 & 14 & - & 22 \\
\hline Coniophora arida* & 2.7 & - & 56 & 22 & 22 & - & 9 \\
\hline Phellinus chrysoloma & 2.7 & 10 & 33 & 37 & 20 & - & 30 \\
\hline Phellinus ferrugineofuscus & 2.7 & 3 & 38 & 41 & 17 & - & 29 \\
\hline Gloeoporus taxicola & 2.8 & - & 41.5 & 41.5 & 17 & - & 12 \\
\hline Fomitopsis rosea* & 2.8 & - & 42 & 41 & 16 & 1 & 79 \\
\hline Coniophora olivacea* & 2.8 & 24 & 10 & 28 & 38 & - & 29 \\
\hline Botryobasidium obtusisporum & 2.8 & 20 & 20 & 20 & 40 & - & 5 \\
\hline Amylocystis lapponica* & 2.9 & - & 38 & 36 & 26 & - & 42 \\
\hline Antrodiella citrinella & 3 & - & - & 100 & - & - & 1 \\
\hline Columnocystis abietina* & 3 & 3,5 & 18 & 53.5 & 25 & - & 28 \\
\hline Dichomitus squalens & 3 & - & - & 100 & - & - & 1 \\
\hline Gelatoporia subvermispora & 3 & - & - & 100 & - & - & 1 \\
\hline Hyphoderma praetermissum & 3 & - & 4 & 14 & 43 & - & 7 \\
\hline Hyphodontia hastata & 3 & - & - & 100 & - & - & 1 \\
\hline Lentaria epichnoa & 3 & - & - & 100 & - & - & 1 \\
\hline Lentinellus castoreus & 3 & - & - & 100 & - & - & 1 \\
\hline Omphalina oniscus & 3 & - & - & 100 & - & - & 1 \\
\hline Phanerochaete sordida & 3 & - & - & 100 & - & - & 1 \\
\hline Phellinus viticola & 3 & - & 27 & 46 & 27 & - & 11 \\
\hline Tomentella ellisii & 3 & - & - & 100 & - & - & 1 \\
\hline Vararia racemosa subsp. lapponica & 3 & - & - & 100 & - & - & 1 \\
\hline
\end{tabular}


Table 14. Contd.

\begin{tabular}{|c|c|c|c|c|c|c|c|}
\hline \multirow[t]{2}{*}{ Species } & \multicolumn{7}{|c|}{ Decay stage } \\
\hline & mean & 1 & 2 & 3 & 4 & 5 & n \\
\hline Amylostereum chailletii & 3.1 & 9 & 9 & 46 & 36 & - & 11 \\
\hline Trichaptum laricinum & 3.2 & - & 11 & 67 & 11 & 11 & 9 \\
\hline Aleurodiscus lividocoeruleus & 3.3 & - & - & 67 & 33 & - & 3 \\
\hline Hyphodontia alutaria & 3.3 & - & 33 & 33 & - & 33 & 3 \\
\hline Antrodia serialis & 3.4 & - & 7 & 48 & 45 & - & 27 \\
\hline Mycena epipterygia & 3.4 & - & 11 & 33 & 56 & - & 9 \\
\hline Laurilia sulcata & 3.5 & - & 13 & 30 & 48 & 9 & 23 \\
\hline Tubulicrinis calothrix & 3.5 & - & - & 54 & 46 & - & 13 \\
\hline Athelia epiphylla & 3.5 & - & - & 50 & 50 & - & 2 \\
\hline Ceraceomyces sublaevis & 3.5 & - & - & 50 & 50 & - & 2 \\
\hline Phanerochaete velutina & 3.5 & - & - & 50 & 50 & - & 2 \\
\hline Skeletocutis kuehneri & 3.5 & - & - & 50 & 50 & - & 2 \\
\hline Tubulicrinis subulatus & 3.5 & - & - & 50 & 50 & - & 2 \\
\hline Botryobasidium subcoronatum & 3.5 & - & 20 & 27 & 33 & 20 & 15 \\
\hline Antrodiella parasitica & 3.6 & - & - & 42 & 58 & - & 12 \\
\hline Oligoporus sericeomollis & 3.6 & - & - & 40 & 60 & - & 5 \\
\hline Tubulicrinis borealis & 3.6 & - & 20 & - & 80 & - & 5 \\
\hline Hyphodontia pallidula & 3.7 & - & - & 33 & 67 & - & 3 \\
\hline Mucronella calva & 3.7 & - & - & 33 & 67 & - & 3 \\
\hline Piloderma byssinum & 3.7 & 11 & 11 & - & 56 & 22 & 9 \\
\hline Tomentellopsis echinospora & 3.7 & - & - & 33 & 67 & - & 3 \\
\hline Trechispora farinacea & 3.7 & - & - & 33 & 67 & - & 3 \\
\hline Phanerochaete laevis & 3.8 & - & - & 50 & 25 & 25 & 4 \\
\hline Dichostereum granulosum & 3.8 & - & - & 20 & 80 & - & 5 \\
\hline Phellinus nigrolimitatus & 3.8 & - & 4 & 23 & 62 & 11 & 56 \\
\hline Botryobasidium botryosum & 3.8 & - & - & 33 & 50 & 17 & 6 \\
\hline Asterodon ferruginosus & 3.9 & - & - & 29 & 57 & 14 & 7 \\
\hline Phlebiella vaga & 3.9 & - & - & 43 & 28.5 & 28.5 & 14 \\
\hline Crepidotus subsphaerosporus & 4 & - & - & - & 100 & - & 1 \\
\hline Hymenochaete fuliginosa & 4 & - & - & 50 & - & 50 & 2 \\
\hline Hyphodontia breviseta & 4 & - & - & 26.5 & 47 & 26.5 & 15 \\
\hline Hypochnicium albostramineum & 4 & - & - & - & 100 & - & 2 \\
\hline Leucogyrophana romellii ${ }^{*}$ & 4 & - & - & - & 100 & - & 1 \\
\hline Phanerochaete sanguinea & 4 & - & - & 25 & 50 & 25 & 4 \\
\hline Amylocorticium cebennense* & 4 & - & - & - & 100 & - & 1 \\
\hline Ceraceomerulius serpens & 4 & - & - & - & 100 & - & 1 \\
\hline Fibulomyces septentrionalis & 4 & - & - & - & 100 & - & 1 \\
\hline Globulicium hiemale & 4 & - & - & - & 100 & - & 1 \\
\hline Conferticium ochraceum & 4 & - & - & - & 100 & - & 1 \\
\hline Hyphoderma cremeoalbum & 4 & - & - & - & 100 & - & 2 \\
\hline Hyphodontia alutacea & 4 & - & - & - & 100 & - & 2 \\
\hline H. cineracea & 4 & - & - & - & 100 & - & 1 \\
\hline Ischnoderma benzoinum & 4 & - & - & - & 100 & - & 2 \\
\hline Kavinia alboviridis & 4 & - & - & - & 100 & - & 1 \\
\hline Phlebiella pseudotsugae & 4 & - & - & 25 & 50 & 25 & 4 \\
\hline Piloporia sajanensis & 4 & - & - & - & 100 & - & 1 \\
\hline Postia placenta* & 4 & - & - & - & 100 & - & 1 \\
\hline Protodontia piceicola & 4 & - & - & - & 100 & - & 1 \\
\hline Pseudotomentella nigra & 4 & - & - & 50 & - & 50 & 2 \\
\hline$P$. tristis & 4 & - & - & 50 & - & 50 & 2 \\
\hline Scytinostromella nannfeldtii & 4 & - & - & - & 100 & - & 1 \\
\hline
\end{tabular}


Table 14. Contd.

\begin{tabular}{|c|c|c|c|c|c|c|c|}
\hline \multirow[t]{2}{*}{ Species } & \multicolumn{7}{|c|}{ Decay stage } \\
\hline & mean & 1 & 2 & 3 & 4 & 5 & $\mathrm{n}$ \\
\hline Sistotrema sernanderi & 4 & - & - & - & 100 & - & 1 \\
\hline Skeletocutis sp. 1 & 4 & - & - & - & 100 & - & 1 \\
\hline Tomentella bryophila & 4 & - & - & - & 100 & - & 1 \\
\hline T. stuposa & 4 & - & - & - & 100 & - & 1 \\
\hline T. sublilacina & 4 & - & - & - & 100 & - & 2 \\
\hline Trechispora subsphaerospora & 4 & - & - & - & 100 & - & 1 \\
\hline Tricholomopsis decora & 4 & - & - & - & 100 & - & 1 \\
\hline Tubulicrinis accedens & 4 & - & - & - & 100 & - & 1 \\
\hline Hyphoderma argillaceum & 4.1 & - & - & - & 87.5 & 12.5 & 8 \\
\hline Skeletocutis stellae & 4.2 & - & - & 17 & 50 & 33 & 6 \\
\hline Hyphodontia aspera & 4.2 & - & - & 20 & 40 & 40 & 5 \\
\hline Gloiothele citrina & 4.2 & - & - & 20 & 40 & 40 & 5 \\
\hline Pseudotomentella mucidula & 4.3 & - & - & - & 67 & 33 & 3 \\
\hline Serpula himantioides* & 4.3 & - & - & - & 67 & 33 & 3 \\
\hline Piloderma croceum & 4.4 & - & - & 15 & 30 & 55 & 20 \\
\hline Tylospora fibrillosa & 4.4 & - & - & 8 & 42 & 50 & 12 \\
\hline Ceraceomyces borealis & 4.5 & - & - & - & 50 & 50 & 2 \\
\hline Botryobasidium angustisporum & 5 & - & - & - & - & 100 & 2 \\
\hline Byssocorticium terrestre & 5 & - & - & - & - & 100 & 1 \\
\hline Resinicium furfuraceum & 5 & - & - & - & - & 100 & 1 \\
\hline
\end{tabular}

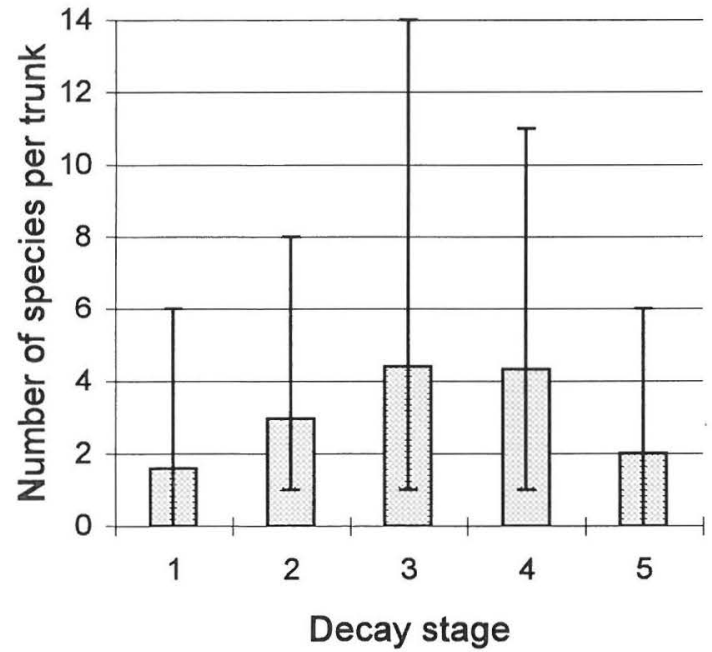

Fig. 5. The number of Basidiomycete species (meantrange) per trunk on fallen spruce (Picea abies subsp. obovata) trunks $(n=320)$ at different stages of decay (1-5).

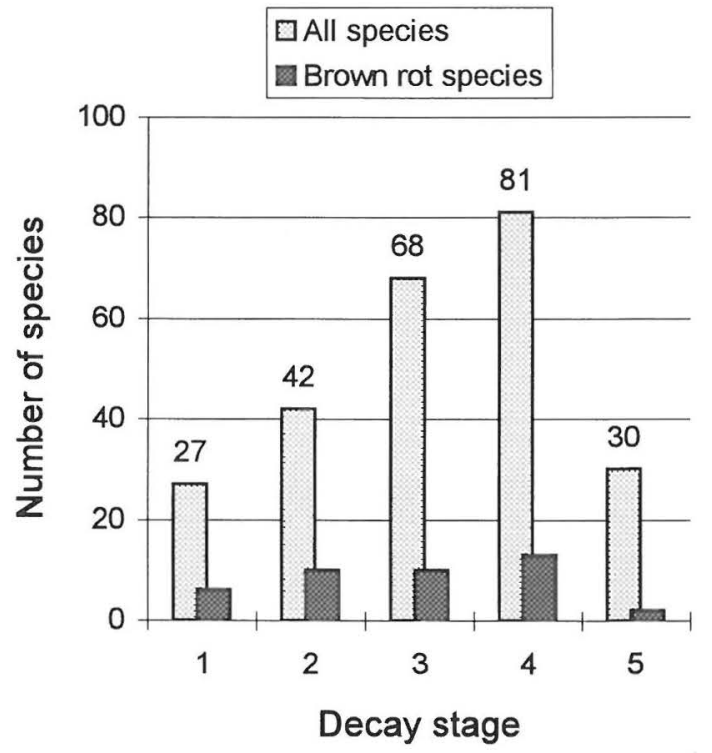

Fig. 6. Total number of Basidiomycete species on fallen spruce (Picea abies subsp. obovata) trunks $(\mathrm{n}=320)$ at different stages of decay (1-5). 


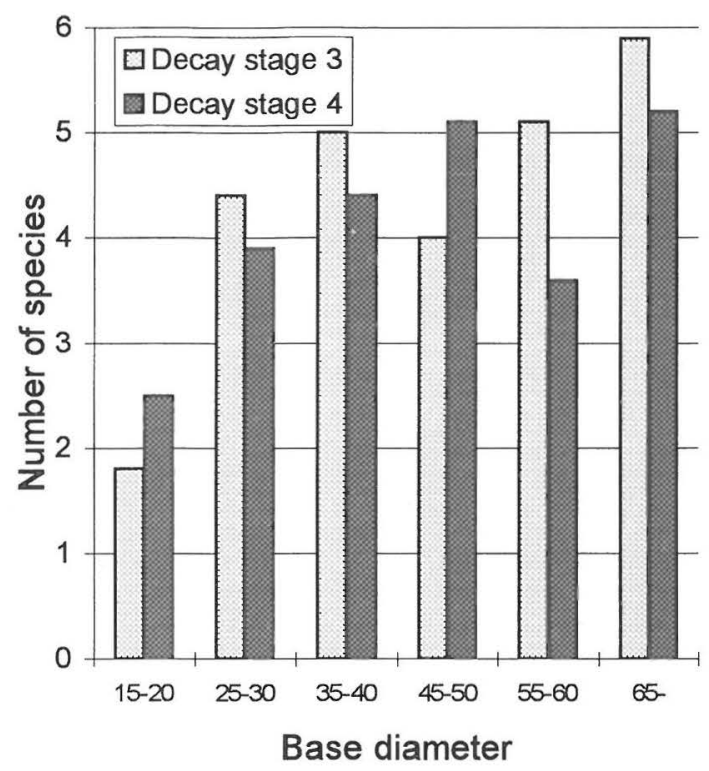

Fig. 7. Number of Basidiomycete species (mean) per trunk on fallen spruce (Picea abies subsp. obovata) trunks in different base diameter $(\mathrm{cm})$ classes.

number of species per trunk showed a weak positive correlation (not tested) with the base diameter of the trunk (Fig. 7). However, as shown in Table 17, the species compositions were very different on thin and thick trunks.

The number of species if brown rot fungi increased from decay stage 1 ( 6 species) to decay stage 4 (13 species), while the proportion of brown rot species decreased from $22 \%$ (D.S. 1) to $7 \%$ (D.S. 5) (Fig. 8). However, $50 \%$ of all the spruce trunks were inhabited by at least one brown rot fungus. At decay stage 1, $23 \%$ of the

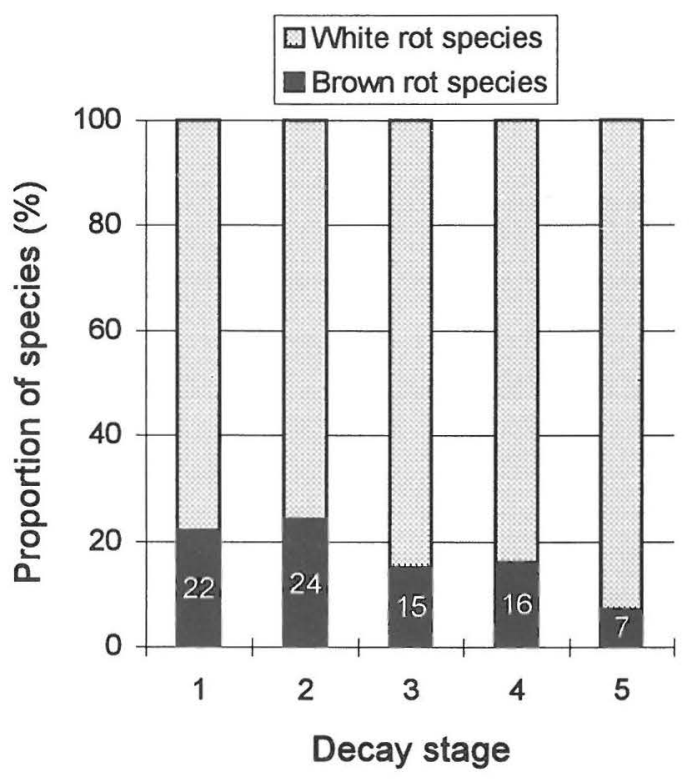

Fig. 8. Proportions of brown and white rot fungi on fallen spruce (Picea abies subsp. obovata) trunks $(\mathrm{n}=320)$ at different stages of decay (1-5).

sample trunks were inhabited by a brown-rotter, while $83 \%$ of the trunks at stage 3 harboured at least one such species (Fig. 9). On trunks at late stages of decay (D.S. 4-5) it was mostly white rot fungi that predominated.

Almost one third $(29.7 \%)$ of all the observations ( $n=1016$ ) of fungi on spruce trunks were of a brown-rot species. The proportion of brown rot fungi was highest on trunks of decay stages 2 and 3 (Fig. 10): $42 \%$ of the observations at stage 2 and $38 \%$ at stage 3 represented a brown rot fungus. Their proportion was lowest $(6 \%)$ at decay stage 5 .

Table 15. Values of Sørensen's Quotient of Similarity applied in comparing the species compositions of wood-inhabiting fungi on trunks of Picea abies subsp. obovata $(n=320)$ at different stages of decay $(1-5)$. Figures given in parentheses show the number of species common to the two decay stages.

\begin{tabular}{|c|c|c|c|c|c|c|c|}
\hline Decay stage & & 1 & 2 & & 3 & 4 & 5 \\
\hline 1 & 1.000 & & & & & & \\
\hline 2 & 0.551 & (19) & 1.000 & & & & \\
\hline 3 & 0.274 & (13) & $0.527 \quad(29)$ & 1.000 & & & \\
\hline 4 & 0.222 & (12) & 0.423 (26) & 0.685 & (51) & 1.000 & \\
\hline 5 & 0.070 & (2) & 0.222 & 0.449 & (22) & $0.414 \quad(23)$ & 1.000 \\
\hline
\end{tabular}




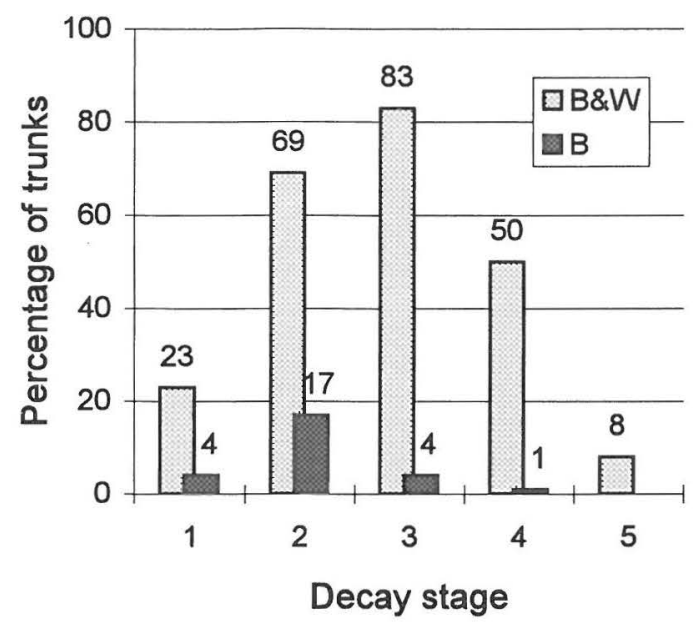

Fig. 9. Percentage of spruce (Picea abies subsp. obovata) trunks $(n=320)$ at different stages of decay $(1-5)$ inhabited by brown rot fungi; $\mathrm{B}=$ percentage of trunks inhabited solely by brown rot fungi; $\mathrm{B} \& \mathrm{~W}=$ percentage of trunks inhabited by both brown and white rot fungi.

Organization. The occurrence of fungal species on sample trunks of spruce varied according to the basal diameter of the trunks. Climacocystis borealis, Laurilia sulcata, Phlebia centrifuga and

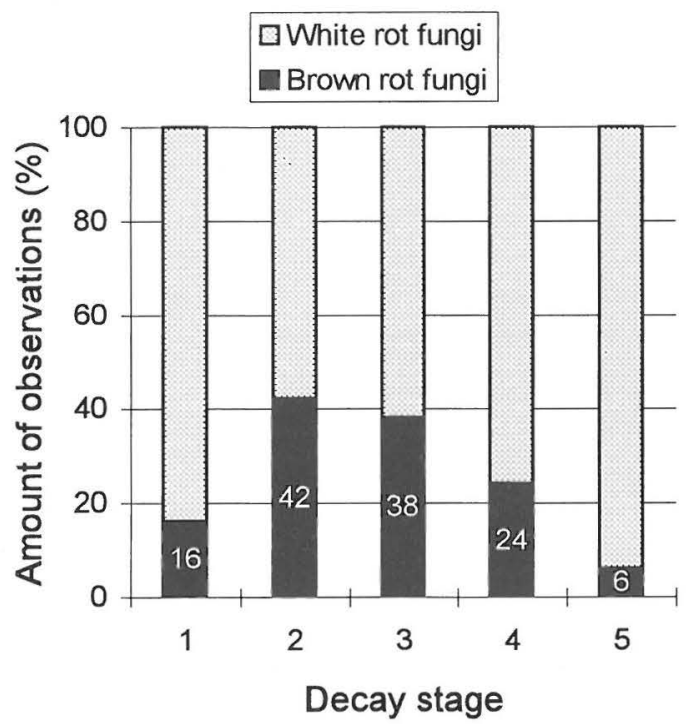

Fig. 10. Observations of brown rot fungi on fallen spruce (Picea abies subsp. obovata) trunks $(\mathrm{n}=320)$ at different stages of decay (1-5) as percentage of all observations.
Table 16. The occurrence of Basidiomycetes on fallen trunks of Picea abies subsp. obovata $(\mathrm{n}=320)$, arranged according to the cover of bark on the trunks. Species recorded less than five times have been omitted. Brown rot species are marked with *; Bark $=$ the mean of the bark cover on the trunks (in \%) \pm one standard deviation; $\mathrm{n}=$ number of observations.

\begin{tabular}{|c|c|c|}
\hline Species & Bark & $\mathrm{n}$ \\
\hline Peniophora septentrionalis & $98 \pm 5$ & 5 \\
\hline Climacocystis borealis & $96 \pm 8$ & 7 \\
\hline Stereum sanguinolentum & $95 \pm 17$ & 40 \\
\hline Onnia leporina & $87 \pm 29$ & 22 \\
\hline Phellinus chrysoloma & $81 \pm 25$ & 24 \\
\hline Exidia saccharina & $80 \pm 35$ & 7 \\
\hline Hyphoderma praetermissum & $79 \pm 11$ & 7 \\
\hline Leptoporus mollis* & $78 \pm 36$ & 9 \\
\hline Phellinus ferrugineofuscus & $77 \pm 25$ & 24 \\
\hline Phlebia centrifuga & $75 \pm 29$ & 44 \\
\hline Skeletocutis odora & $75 \pm 22$ & 22 \\
\hline Trichaptum laricinum & $75 \pm 27$ & 8 \\
\hline T. fuscoviolaceum & $74 \pm 42$ & 10 \\
\hline Peniophora pithya & $72 \pm 33$ & 6 \\
\hline Trichaptum abietinum & $71 \pm 30$ & 41 \\
\hline Coniophora arida* & $69 \pm 30$ & 9 \\
\hline Gloeoporus taxicola & $66 \pm 37$ & 12 \\
\hline Fomitopsis pinicola & $66 \pm 33$ & 35 \\
\hline Gloeophyllum sepiarium & $65 \pm 31$ & 32 \\
\hline Coniophora olivacea & $61 \pm 37$ & 26 \\
\hline Fomitopsis rosea & $59 \pm 33$ & 78 \\
\hline Antrodia serialis: & $52 \pm 30$ & 24 \\
\hline Amylostereum chailletii & $51 \pm 29$ & 11 \\
\hline Phellinus viticola & $49 \pm 26$ & 11 \\
\hline Mycena epipterygia & $49 \pm 33$ & 9 \\
\hline Antrodiella parasitica & $49 \pm 25$ & 12 \\
\hline Amylocystis lapponica* & $49 \pm 28$ & 40 \\
\hline Botryobasidium botryosum & $48 \pm 37$ & 5 \\
\hline B. subcoronatum & $46 \pm 37$ & 15 \\
\hline Asterodon ferruginosus & $45 \pm 39$ & 6 \\
\hline Columnocystis abietina & $44 \pm 31$ & 29 \\
\hline Dichostereum granulosum & $44 \pm 44$ & 5 \\
\hline Laurilia sulcata & $40 \pm 35$ & 24 \\
\hline Oligoporus sericeomollis* & $34 \pm 28$ & 5 \\
\hline Tubulicrinis calothrix & $34 \pm 28$ & 13 \\
\hline Piloderma byssinum & $31 \pm 33$ & 9 \\
\hline Skeletocutis stellae & $29 \pm 27$ & 6 \\
\hline Phellinus nigrolimitatus & 29_31 & 55 \\
\hline Tubulicrinis borealis & $28 \pm 33$ & 5 \\
\hline Phlebiella vaga & $26 \pm 32$ & 13 \\
\hline Hyphodontia breviseta & $23 \pm 32$ & 15 \\
\hline Hyphodontia aspera & $23 \pm 30$ & 6 \\
\hline Piloderma croceum & $22 \pm 33$ & 21 \\
\hline Tylospora fibrillosa & $22 \pm 25$ & 13 \\
\hline Hyphoderma argillaceum & $16 \pm 18$ & 8 \\
\hline Gloiothele citrina & $10 \pm 18$ & 6 \\
\hline
\end{tabular}


Table 17. Basidiomycetes found on fallen trunks of Picea abies subsp. obovata, arranged according to the base diameter of the trunks. Species recorded less than five times have been omitted. Brown rot species are marked with *; Frb diam $=$ diameter of the trunk at the place of basidiocarps; $\mathrm{n}=$ number of observations.

\begin{tabular}{|c|c|c|c|c|c|}
\hline \multirow[t]{2}{*}{ Species } & \multicolumn{2}{|c|}{ Base diam } & \multicolumn{2}{|c|}{ Frb diam } & \multirow[t]{2}{*}{$\mathrm{n}$} \\
\hline & mean & range & mean & range & \\
\hline Climacocystis borealis & 64 & $50-90$ & 63 & $45-90$ & 7 \\
\hline Skeletocutis odora & 52 & $30-80$ & 38 & $20-70$ & 24 \\
\hline Laurilia sulcata & 50 & $20-90$ & 42 & $20-70$ & 27 \\
\hline Tubulicrinis calothrix & 49 & $30-80$ & 23 & $10-60$ & 13 \\
\hline Phlebia centrifuga & 48 & $25-90$ & 38 & $10-75$ & 51 \\
\hline Gloeoporus taxicola & 47 & $20-80$ & 24 & $5-40$ & 14 \\
\hline Mycena epipterygia & 47 & $30-75$ & 33 & $20-45$ & 10 \\
\hline Serpula himantioides & 47 & $35-70$ & 31 & $20-50$ & 5 \\
\hline Asterodon ferruginosus & 46 & $35-60$ & 29 & $10-55$ & 7 \\
\hline Hyphoderma argillaceum & 46 & $30-70$ & 35 & $10-65$ & 8 \\
\hline Botryobasidium subcoronatum & 45 & $20-80$ & 32 & $5-80$ & 16 \\
\hline Fomitopsis pinicola & 44 & $15-80$ & 36 & $10-70$ & 40 \\
\hline Coniophora arida* & 43 & $15-90$ & 27 & $10-80$ & 10 \\
\hline Fomitopsis rosea* & 43 & $15-90$ & 32 & $10-70$ & 110 \\
\hline Leptoporus mollis* & 43 & $25-75$ & 28 & $20-40$ & 10 \\
\hline Phellinus nigrolimitatus & 43 & $15-80$ & 40 & $15-80$ & 62 \\
\hline Piloderma byssinum & 43 & $30-60$ & 25 & $5-50$ & 10 \\
\hline Hyphodontia breviseta & 42 & $30-65$ & 28 & $10-50$ & 15 \\
\hline Phellinus ferrugineofuscus & 42 & $25-75$ & 28 & $5-65$ & 37 \\
\hline Skeletocutis stellae & 42 & $30-60$ & 35 & $25-55$ & 6 \\
\hline Antrodia serialis & 41 & $25-80$ & 19 & $10-35$ & 31 \\
\hline Amylocystis lapponica & 41 & $25-80$ & 31 & $10-60$ & 56 \\
\hline Dichostereum granulosum & 41 & $35-50$ & 35 & $15-50$ & 5 \\
\hline Tubulicrinis borealis & 41 & $25-60$ & 30 & $15-55$ & 5 \\
\hline Coniophora olivacea & 40 & $15-80$ & 24 & $10-55$ & 28 \\
\hline Amylostereum chailletii & 40 & $10-60$ & 32 & $10-55$ & 12 \\
\hline Tylospora fibrillosa & 40 & $25-70$ & 24 & $5-45$ & 14 \\
\hline Phellinus chrysoloma & 40 & $10-90$ & 31 & $5-60$ & 38 \\
\hline Columnocystis abietina* & 40 & $20-70$ & 18 & $10-50$ & 30 \\
\hline Hyphoderma praetermissum & 39 & $25-60$ & 27 & $10-45$ & 7 \\
\hline Antrodiella parasitica & 38 & $15-80$ & 27 & $10-80$ & 12 \\
\hline Exidia saccharina & 38 & $20-50$ & 30 & $10-50$ & 7 \\
\hline Phellinus viticola & 38 & $15-70$ & 25 & $5-40$ & 13 \\
\hline Phlebiella vaga & 38 & $25-60$ & 20 & $5-50$ & 15 \\
\hline Piloderma croceum & 38 & $20-55$ & 22 & $5-50$ & 20 \\
\hline Onnia leporina & 37 & $20-55$ & 36 & $20-55$ & 25 \\
\hline Trichaptum abietinum & 37 & $15-80$ & 19 & $5-50$ & 57 \\
\hline Oligoporus sericeomollis* & 37 & $30-50$ & 23 & $10-50$ & 5 \\
\hline Phanerochaete sanguinea & 37 & $15-55$ & 16 & $5-15$ & 5 \\
\hline Gloeophyllum sepiarium* & 35 & $15-80$ & 19 & $10-45$ & 39 \\
\hline Peniophora pithya & 33 & $20-40$ & 21 & $10-30$ & 6 \\
\hline Stereum sanguinolentum & 33 & $20-55$ & 30 & $5-55$ & 41 \\
\hline Trichaptum fuscoviolaceum & 33 & $20-55$ & 23 & $10-35$ & 12 \\
\hline T. laricinum & 32 & $10-40$ & 29 & $5-40$ & 17 \\
\hline Peniophora septentrionalis & 30 & $20-45$ & 22 & $5-40$ & 5 \\
\hline Hyphodontia aspera & 29 & $25-35$ & 26 & $20-35$ & 5 \\
\hline
\end{tabular}


Skeletocutis odora are examples of species that mostly inhabited large trunks, whereas, Gloeophyllum sepiarium, Peniophora septentrionalis, Stereum sanguinolentum and Trichaptum laricinum occupied fairly thin trunks having a base diameter mostly less than $35 \mathrm{~cm}$ (Table 17). Amylocystis lapponica, Fomitopsis rosea, Phellinus chrysoloma, $P$. nigrolimitatus and many other species seemed to prefer medium-size trunks.

Species recorded in the basal parts of the trunks were usually not found at all in the crown. On the other hand, fungi occupying the top third of the trunks were usually absent from the basal parts. The Relative Locality index (R.L.) was used to describe the average proximal vs. distal distribution of the fungi on the sample trunks and the values are summarized in Table 18. Onnia leporina, Climacocystis borealis, Stereum sanguinolentum and Trichaptum laricinum (R.L. values 0.90-0.98) were restricted to the base and were seldom found elsewhere. The basal third of the trunks was also preferred by Fomitopsis pinicola, Skeletocutis odora, Laurilia sulcata, Leptoporus mollis, Dichostereum granulosum and Phellinus nigrolimitatus (R.L. values 0.80-0.88). The middle parts of the trunks were occupied by a great variety of species, which, however, usually had different optimum values for their longitudinal locations. Phanerochaete sanguinea, Columnocystis abietina, Trichaptum abietinum and Antrodia serialis (R.L. values $0.46-0.49$ ) were usually restricted to the crown. A clear preference for the thinnest parts of the trunks was also characteristic of Tubulicrinis calothrix, Gloeoporus taxicola and Gloeophyllum sepiarium. Most of the species inhabiting middle parts and the crown were saprotrophs, while those restricted to the base included important butt rot-causing pathogens.

Table 18. Values of the Relative Locality index (R.L.) applied in describing and comparing the average proximal vs. distal location of the wood-inhabiting fungi on fallen trunks of Picea abies subsp. obovata $(\mathrm{n}=320)$. Brown rot species are marked with *; $n=$ number of observations.

\begin{tabular}{|c|c|c|c|c|c|}
\hline Species & R.L. & $\mathrm{n}$ & Species & R.L. & $\mathrm{n}$ \\
\hline Onnia leporina & 0.98 & 23 & Hyphodontia breviseta & 0.70 & 15 \\
\hline Climacocystis borealis & 0.93 & 6 & Coniophora arida* & 0.70 & 9 \\
\hline Skeletocutis stellae & 0.90 & 6 & Coniophora olivacea* & 0.66 & 29 \\
\hline Stereum sanguinolentum & 0.90 & 42 & Phanerochaete laevis & 0.66 & $\begin{array}{r}29 \\
4\end{array}$ \\
\hline Trichaptum laricinum & 0.88 & 10 & Hyphoderma praetermissum & 0.66 & 7 \\
\hline Fomitopsis pinicolat: & 0.83 & 36 & Peniophora pithya & 0.66 & 6 \\
\hline Skeletocutis odora & 0.82 & 20 & $P$. septentrionalis & 0.65 & 5 \\
\hline Laurilia sulcata & 0.81 & 25 & Oligoporus sericeomollis & 0.65 & 4 \\
\hline Leptoporus mollis* & 0.81 & 9 & Tylospora fibrillosa & 0.65 & 12 \\
\hline Dichostereum granulosum & 0.80 & 4 & Mycena epipterygia & 0.64 & 7 \\
\hline Phellinus nigrolimitatus & 0.80 & 55 & Botryobasidium botryosum & 0.62 & 5 \\
\hline Hyphodontia aspera & 0.79 & 5 & Trichaptum fuscoviolaceum & 0.62 & 11 \\
\hline Hyphoderma argillacaeum & 0.79 & 8 & Asterodon ferruginosus & 0.62 & 6 \\
\hline Phlebia centrifuga & 0.77 & 44 & Gloiothele citrina & 0.59 & 5 \\
\hline Amylostereum chailletii & 0.76 & 11 & Piloderma byssinum & 0.56 & 9 \\
\hline Botryobasidium obtusisporum & 0.76 & 5 & Phellinus viticola & 0.55 & 11 \\
\hline Phellinus chrysoloma & 0.76 & 33 & Piloderma croceum & 0.54 & 19 \\
\hline Phlebiella pseudotsugae & 0.75 & 5 & Gloeophyllum sepiarium* & 0.52 & 35 \\
\hline Exidia saccharina & 0.74 & 7 & Gloeoporus taxicola & 0.51 & 12 \\
\hline Amylocystis lapponica* & 0.74 & 40 & Phlebiella vaga & 0.50 & 14 \\
\hline Botryobasidium subcoronatum & 0.72 & 14 & Tubulicrinis calothrix & 0.50 & 13 \\
\hline Fomitopsis rosea* & 0.72 & 66 & Antrodia serialis & 0.49 & 27 \\
\hline Antrodiella parasitica & 0.72 & $\begin{array}{r}12 \\
5\end{array}$ & Trichaptum abietinum & 0.49 & 51 \\
\hline Tubulicrinis borealis & 0.71 & $\begin{array}{r}5 \\
34\end{array}$ & Columnocystis abietina* & 0.47 & 26 \\
\hline Phellinus ferrugineofuscus & 0.70 & 34 & Phanerochaete sanguinea & 0.46 & 5 \\
\hline
\end{tabular}


Sequence, diversity and organization of fungi on pine trunks

Sequence. As on spruce, the compositions of the fungal communities on pine greatly depended on the stage of decomposition of the trunks. Certain species clearly preferred hard, undecayed trunks, while others inhabited strongly decayed trunks with softened wood.

The most frequent fungi on newly uprooted, corticated pine trunks were Stereum sanguinolentum, which was recorded on $33 \%$ of trunks at decay stage 1, and Trichaptum fuscoviolaceum (Table 19). These two species evidently are the first Basidiomycetes to invade pine trunks after their fall. Often they were associated with other white rot fungi such as Hyphoderma setigerum, Exidia saccharina and Phlebiopsis gigantea. Most of the sample trunks at decay stage 2 were already decorticated. Every third one (35.7\%) was inhabited by Amyloporia xantha, which was the commonest and evidently quantitatively the most important decayer. A. xantha was also the most important decayer at decay stage 3 . Other typical fungi of decay stage 2 were Antrodia primaeva, which preferred fairly large and charred trunks, and Fomitopsis pinicola and Trichaptum abietinum, which often shared the same trunks. Phellinus viticola and Antrodia sinuosa favoured both the decay stages 2 and 3 . Almost all species that were frequently recorded on trunks at decay stages 2 and 3 were brown rot fungi. In addition to Amyloporia xantha, also Postia lateritia, P. hibernica, Chaetodermella luna and Antrodia albobrunnea were frequently found on trunks at decay stage 3. Strongly decayed and already soft pine trunks (D.S. 4) were characterized by the presence of three species. Antrodia albobrunnea and Skeletocutis lenis were dominant decomposers, recorded, respectively, on $30 \%$ and $22 \%$ of the sample trunks at decay stage 4 . Piloderma croceum was recorded on $28 \%$ of trunks. Other abundant corticiaceous fungi at this stage of decay were Phlebiella vaga, Botryobasidium botryosum, $B$. subcoronatum, Leucogyrophana romellii, Resinicium furfuraceum and Trechispora farinacea. The strongly decomposed remnants of pine trunks (D.S. 5) bore still fruiting basidiomes of Skeletocutis lenis and Antrodia albobrunnea. The former was found on $25 \%$ and the latter on $20 \%$ of trunks at the final stage of decay.

A characteristic feature of old pine forests of northern Finland is the large number of decorticated trunks. Pines very often reach an age of 100-300 years, die and lose their bark but then remain standing for decades. After falling these silvery grey decorticated trunks with dry and resin-rich wood, kelo trees in Finnish, harboured almost totally different mycoflora than the corticated trunks of trees that died in a more usual way (Table 20). Almost three fourths (73.1\%) of the species on pine trunks were recorded on totally decorticated trunks only. Fresh windfalls hosted only a few species that were also found on extensively decayed, decorticated trunks at the late stages of decomposition. On the other hand, only $12 \%$ of species that inhabited sample trunks at decay stages 3-5 were also recorded on the trunks at stage 1 (Table 21).

Diversity. The number of species on a single pine trunk ranged from 0 (D.S. 1 and 5) to 13 (D.S. 4) (Fig. 11). The mean species number per trunk was 2.1. If the almost completely decayed trunks (D.S. 5) are excluded, the number of species increased sharply with the decay stage. The trunks at decay stage 1 harboured only 11 species, whereas altogether 68 species were recorded on trunks at decay stage 3 (Fig. 12). The number of species per trunk showed a weak positive correlation (not tested) with the base diameter of the trunk (Fig. 13). However, as shown in Table 22, the species compositions were very different on thin and thick trunks.

In all, 22 brown rot fungi were recorded on pine trunks. The number of brown rot species increased from decay stage 1 ( 4 species) to decay stage 3 ( 20 species), while their proportion of all species at a particular stage decreased evenly from decay stage 1 ( $36 \%$ of the species) to decay stage 4 (19\%) (Fig. 14). One out of four (23.9\%) trunks was inhabited only by a brown rot fungus, and over one half $(52.7 \%)$ of all trunks were inhabited by at least one brown rot fungus. At decay stage 1 only $10 \%$ of the sample trunks were inhabited by a brown-rotter, while at stage 3 as many as $77 \%$ of the trunks harboured a brown rot fungus (Fig. 15).

Over one third (37.2\%) of all the observations $(\mathrm{n}=914)$ of fungi on pine trunks were brown rot species. Their proportion (persentage of all observations) was highest on trunks at decay stages 2 and 3 (Fig. 16): 51\% and at stage 2 and $48 \%$ at stage 3 . The proportion was lowest $(10 \%$ of all observations) on trunks at decay stage 1 . 
Table 19. List of Basidiomycetes found on fallen trunks of Pinus sylvestris in northeastern Finland. Species are arranged according to their preference for stage of trunk decay ( $1-5, \mathrm{n}=$ number of observations). Species that cause brown rot are marked with *.

\begin{tabular}{|c|c|c|c|c|c|c|c|}
\hline \multirow[t]{2}{*}{ Species } & \multicolumn{7}{|c|}{ Decay stage } \\
\hline & mean & 1 & 2 & 3 & 4 & 5 & $\mathrm{n}$ \\
\hline Coniophora arida* & 1 & 100 & - & - & - & - & 1 \\
\hline Panellus mitis & 1 & 100 & - & - & - & - & 4 \\
\hline Peniophora pini & 1 & 100 & - & - & - & - & 1 \\
\hline Exidia saccharina & 1.2 & 92 & - & 8 & - & - & 13 \\
\hline Phlebiopsis gigantea & 1.2 & 82 & 18 & - & - & - & 11 \\
\hline Stereum sanguinolentum & 1.2 & 81 & 17 & 2 & - & - & 41 \\
\hline Gloeophyllum sepiarium* & 1.3 & 75 & 25 & - & - & - & 4 \\
\hline Hyphoderma setigerum & 1.3 & 72 & 28 & - & - & - & 18 \\
\hline Trichaptum fuscoviolaceum & 1.5 & 65 & 29 & 6 & - & - & 34 \\
\hline Ischnoderma benzoinum & 2 & - & 100 & - & - & - & 1 \\
\hline Hyphoderma argillaceum & 2 & - & 100 & - & - & - & 1 \\
\hline Hyphodontia hastata & 2 & - & 100 & - & - & - & 1 \\
\hline H. subalutacea & 2 & - & 100 & - & - & - & 1 \\
\hline Skeletocutis amorpha & 2 & - & 100 & - & - & - & 1 \\
\hline Tubulicrinis borealis & 2 & - & 100 & - & - & - & 1 \\
\hline Fomitopsis pinicola* & 2.2 & - & 78 & 22 & - & - & 9 \\
\hline Trichaptum abietinum & 2.4 & - & 70 & 20 & 10 & - & 10 \\
\hline Athelia cf. acrospora & 2.5 & - & 50 & 50 & - & - & 2 \\
\hline Gloeoporus taxicola & 2.5 & - & 50 & 50 & - & - & 2 \\
\hline Tomentella radiosa & 2.5 & - & 50 & 50 & - & - & 2 \\
\hline Amyloporia xantha* & 2.6 & 4 & 51 & 30 & 13 & 2 & 70 \\
\hline Gloeophyllum protractum* & 2.6 & - & 44 & 56 & - & - & 9 \\
\hline Antrodia primaeva* & 2.6 & - & 48 & 48 & 4 & - & 23 \\
\hline Sistotrema muscicola & 2.6 & - & 57 & 29 & 14 & - & 7 \\
\hline Chaetodermella luna* & 2.8 & - & 39 & 48 & 10 & 3 & 31 \\
\hline Antrodia sinuosa* & 2.8 & 6 & 35 & 35 & 24 & - & 17 \\
\hline Antrodiella parasitica & 2.8 & - & 40 & 40 & 20 & - & 5 \\
\hline Junghuhnia luteoalba & 2.8 & - & 25 & 75 & - & - & 4 \\
\hline Trichaptum laricinum & 2.8 & - & 20 & 80 & - & - & 5 \\
\hline Phellinus viticola & 2.9 & - & 36 & 39 & 21 & 4 & 28 \\
\hline Aleurodiscus lividocoeruleus & 3 & - & 50 & - & 50 & - & 2 \\
\hline Amylocorticium cebennense* & 3 & - & - & 100 & - & - & 1 \\
\hline Amylocystis lapponica* & 3 & - & - & 100 & - & - & 2 \\
\hline Antrodia infirma* & 3 & - & - & 100 & - & - & 4 \\
\hline Botryobasidium obtusisporum & 3 & - & - & 100 & - & - & 1 \\
\hline Byssocorticium terrestre & 3 & - & - & 100 & - & - & 1 \\
\hline Columnocystis abietina* & 3 & - & - & 100 & - & - & 1 \\
\hline Fomitopsis rosea & 3 & - & - & 100 & - & - & 1 \\
\hline Hyphoderma cf. sibiricum & 3 & - & - & 100 & - & - & 1 \\
\hline Hyphodontia aspera & 3 & - & - & 100 & - & - & 2 \\
\hline H. pallidula & 3 & - & - & 100 & - & - & 1 \\
\hline Leptosporomyces fuscoatrus & 3 & - & - & 100 & - & - & 1 \\
\hline Phellinus ferrugineofuscus & 3 & - & - & 100 & - & - & 1 \\
\hline Phlebia cretacea & 3 & - & - & 100 & - & - & 1 \\
\hline Phlebiella borealis & 3 & - & - & 100 & - & - & 1 \\
\hline P. pseudotsugae & 3 & - & - & 100 & - & - & 2 \\
\hline Postia lateritia* & 3 & - & 8 & 83 & 8 & - & 24 \\
\hline Pseudotomentella tristis & 3 & - & - & 100 & - & - & 1 \\
\hline Pseudotomentella sp. 1 & 3 & - & - & 100 & - & - & 1 \\
\hline Serpula himantioides* & 3 & - & - & 100 & - & - & 1 \\
\hline
\end{tabular}


Table 19. Contd.

\begin{tabular}{|c|c|c|c|c|c|c|c|}
\hline \multirow[t]{2}{*}{ Species } & \multicolumn{7}{|c|}{ Decay stage } \\
\hline & mean & 1 & 2 & 3 & 4 & 5 & $\mathrm{n}$ \\
\hline Skeletocutis odora & 3 & - & - & 100 & - & - & 1 \\
\hline Amyloporia crassa* & 3.3 & - & - & 75 & 25 & - & 4 \\
\hline Athelia decipiens & 3.3 & - & - & 75 & 25 & - & 4 \\
\hline Tubulicrinis calothrix & 3.3 & - & 25 & 25 & 50 & - & 4 \\
\hline Postia hibernica* & 3.3 & - & 11 & 55 & 30 & 4 & 27 \\
\hline Hypochnicium albostramineum & 3.3 & - & - & 67 & 33 & - & 3 \\
\hline Botryobasidium botryosum & 3.4 & - & 14 & 41 & 41 & 4 & 29 \\
\hline Antrodia albobrunnea* & 3.4 & - & 2 & 23 & 59 & 16 & 51 \\
\hline Botryobasidium subcoronatum & 3.4 & - & 17 & 26 & 57 & - & 23 \\
\hline Phlebia cornea & 3.4 & - & 14 & 43 & 43 & - & 7 \\
\hline Ceraceomyces borealis & 3.4 & - & 14 & 29 & 57 & - & 7 \\
\hline Sistotremastrum suecicum & 3.5 & - & 18 & 27 & 46 & 9 & 11 \\
\hline Oligoporus sericeomollis* & 3.5 & - & 16 & 21 & 63 & - & 19 \\
\hline Physodontia lundellii & 3.5 & - & - & 50 & 50 & - & 2 \\
\hline Piloderma croceum & 3.6 & - & 11 & 28 & 50 & 11 & 56 \\
\hline Ceraceomyces sublaevis & 3.6 & - & 12.5 & 12.5 & 75 & - & 8 \\
\hline Hyphodontia breviseta & 3.6 & - & - & 37.5 & 62.5 & - & 8 \\
\hline Piloderma olivaceum & 3.7 & - & - & 33 & 67 & - & 6 \\
\hline Skeletocutis subincarnata & 3.7 & - & - & 33 & 67 & - & 3 \\
\hline Coniophora olivacea & 3.7 & - & - & 29 & 71 & - & 7 \\
\hline Phanerochaete sanguinea & 3.7 & - & - & 29 & 71 & - & 7 \\
\hline Tubulicrinis medius & 3.7 & - & - & 29 & 71 & - & 7 \\
\hline Resinicium furfuraceum & 3.7 & - & 5.5 & 28 & 55.5 & 11 & 18 \\
\hline Trechispora farinacea & 3.8 & - & 12.5 & 25 & 37.5 & 25 & 24 \\
\hline Leucogyrophana romellii* & 3.8 & - & 4 & 30 & 48 & 17 & 23 \\
\hline Globulicium hiemale & 3.8 & - & - & 40 & 40 & 20 & 10 \\
\hline Tricholomopsis decora & 3.8 & - & - & 17 & 83 & - & 6 \\
\hline Phlebiella vaga & 3.9 & - & 4 & 26 & 52 & 18 & 27 \\
\hline Asterodon ferruginosus & 4 & - & - & - & 100 & - & 3 \\
\hline Botryobasidium candicans & 4 & - & - & - & 100 & - & 1 \\
\hline Fibulomyces mutabilis & 4 & - & - & - & 100 & - & 1 \\
\hline Hyphoderma pallidum & 4 & - & - & - & 100 & - & 1 \\
\hline H. praetermissum & 4 & - & - & 26.5 & 47 & 26.5 & 15 \\
\hline Hyphodontia alutacea & 4 & - & - & - & 100 & - & 2 \\
\hline H. alutaria & 4 & - & - & - & 100 & - & 1 \\
\hline Leucogyphana mollusca* & 4 & - & - & - & 100 & - & 1 \\
\hline Mucronella bresadolae & 4 & - & - & - & 100 & - & 1 \\
\hline M. calva & 4 & - & - & - & 100 & - & 2 \\
\hline Odonticium romellii & 4 & - & - & - & 100 & - & 5 \\
\hline Phlebia segregata & 4 & - & - & - & 100 & - & 4 \\
\hline Tomentellopsis echinospora & 4 & - & - & - & 100 & - & 1 \\
\hline Tubulicrinis chaetophorus & 4 & - & - & - & 100 & - & 2 \\
\hline T. globisporus & 4 & - & - & - & 100 & - & 3 \\
\hline T. gracillimus & 4 & - & - & - & 100 & - & 1 \\
\hline Gymnopilus penetrans & 4 & - & - & - & 100 & - & 1 \\
\hline Phellinus nigrolimitatus & 4 & - & - & 12.5 & 75 & 12.5 & 8 \\
\hline Skeletocutis jelicii & 4 & - & - & - & 100 & - & 1 \\
\hline S. kuehneri & 4 & - & - & - & 100 & - & 1 \\
\hline S. stellae & 4 & - & - & - & 100 & - & 1 \\
\hline S. lenis & 4.2 & - & 3 & 8 & 61 & 28 & 36 \\
\hline Leptosporomyces galzinii & 4.5 & - & - & - & 50 & 50 & 2 \\
\hline Piloderma byssinum & 4.5 & - & - & - & 50 & 50 & 2 \\
\hline Tubulicrinis effugiens & 4.5 & - & - & - & 50 & 50 & 2 \\
\hline Phlebiella subflavidogrisea & 5 & - & - & - & - & 100 & 1 \\
\hline
\end{tabular}




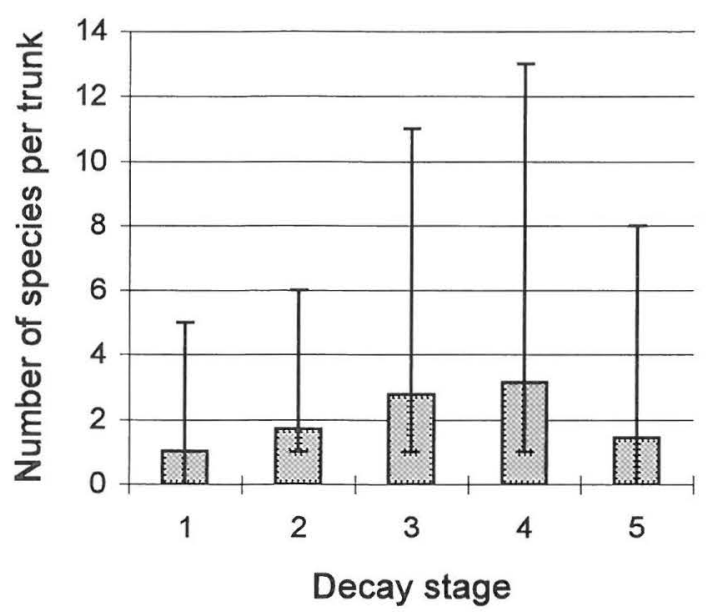

Fig. 11. The number of Basidiomycete species (meantrange) per trunk on fallen pine (Pinus sylvestris) trunks $(n=440)$ at different stages of decay $(1-5)$.

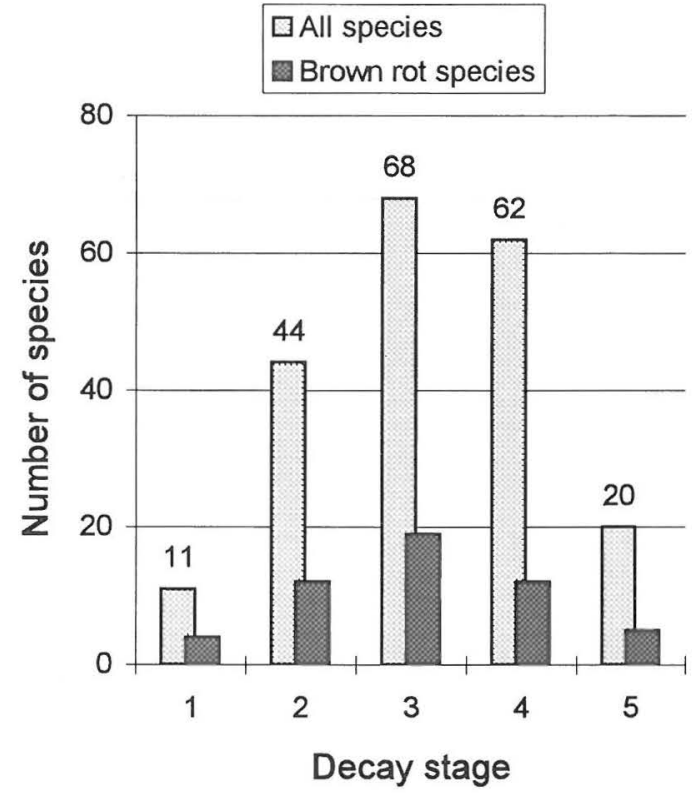

Fig. 12. Total number of Basidiomycete species on fallen pine (Pinus sylvestris) trunks $(\mathrm{n}=440)$ at different stages of decay (1-5).

Table 20. Occurrence of Basidiomycetes on fallen trunks of Pinus sylvestris $(\mathrm{n}=440)$ according to preference for trunks with different amount of bark. Brown rot species are marked with $\%$; Bark $=$ mean of the bark cover (in $\%$ ) \pm one standard deviation; $n=$ number of observations. Species recorded less than five times have been omitted.

\begin{tabular}{|c|c|c|c|c|c|}
\hline Species & Bark & $\mathrm{n}$ & Species & Bark & $\mathrm{n}$ \\
\hline Stereum sanguinolentum & $83 \pm 25$ & 42 & Piloderma croceum & $0.3 \pm 2$ & 54 \\
\hline Trichaptum fuscoviolaceum & $78 \pm 26$ & 34 & Botryobasidium subcoronatum & $0.2 \pm 1$ & 21 \\
\hline Gloeophyllum sepiarium & $78 v 26$ & 5 & Trechispora farinacea & $0.2 \pm 1$ & 24 \\
\hline Exidia saccharina & $70 \pm 29$ & 13 & Sistotrema muscicola & 0 & 7 \\
\hline Phlebiopsis gigantea & $65 \pm 30$ & 13 & Ceraceomyces borealis & 0 & 7 \\
\hline Hyphoderma setigerum & $56 \pm 36$ & 18 & Coniophora olivacea & 0 & 7 \\
\hline Trichaptum abietinum & $43 \pm 33$ & 8 & Globulicium hiemale & 0 & 9 \\
\hline Fomitopsis pinicola* & $41 \pm 32$ & 9 & Gloeophyllum protractum* & 0 & 9 \\
\hline Antrodia sinuosa* & $10 \pm 26$ & 17 & Hyphoderma praetermissum & 0 & 14 \\
\hline A. primaeva* & $10 \pm 24$ & 23 & Leucogyrophana romellii* & 0 & 23 \\
\hline Ceraceomyces sublaevis & $8 \pm 16$ & 8 & Odonticium romellii & 0 & 5 \\
\hline Amyloporia xantha* & $7 \pm 19$ & 70 & Phanerochaete sanguinea & 0 & 6 \\
\hline Hyphodontia breviseta & $3 \pm 5$ & 8 & Phellinus nigrolimitatus & 0 & 8 \\
\hline Phellinus viticola & $3 \pm 10$ & 28 & Phlebia cornea & 0 & 6 \\
\hline Antrodia albobrunnea* & $1 \pm 10$ & 51 & Piloderma olivaceum & 0 & 6 \\
\hline Botryobasidium botryosum & $1 \pm 5$ & 28 & Postia hibernica* & 0 & 27 \\
\hline Postia lateritia* & $1 \pm 3$ & 25 & Resinicium furfuraceum & 0 & 17 \\
\hline Oligoporus sericeomollis* & $1 \pm 5$ & 19 & Sistotremastrum suecicum & 0 & 10 \\
\hline Chaetodermella luna* & $0.3 \pm 2$ & 31 & Skeletocutis lenis & 0 & 35 \\
\hline \multirow{2}{*}{ Phlebiella vaga } & $0.3 \pm 2$ & 28 & Tricholomopsis decora & 0 & 6 \\
\hline & & 20 & Tubulicrinis medius & 0 & 7 \\
\hline
\end{tabular}




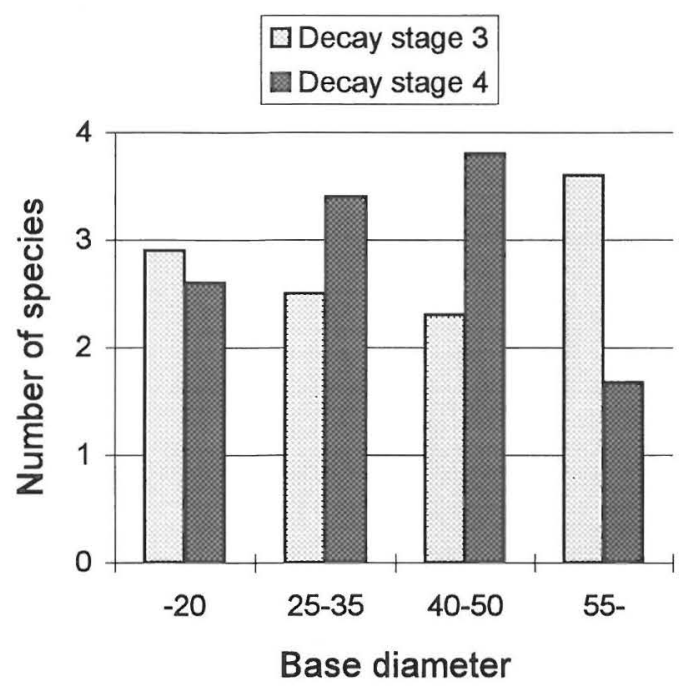

Fig. 13. Number of Basidiomycete species (mean) per trunk on fallen pine (Pinus sylvestris) trunks in different base diameter $(\mathrm{cm})$ classes.

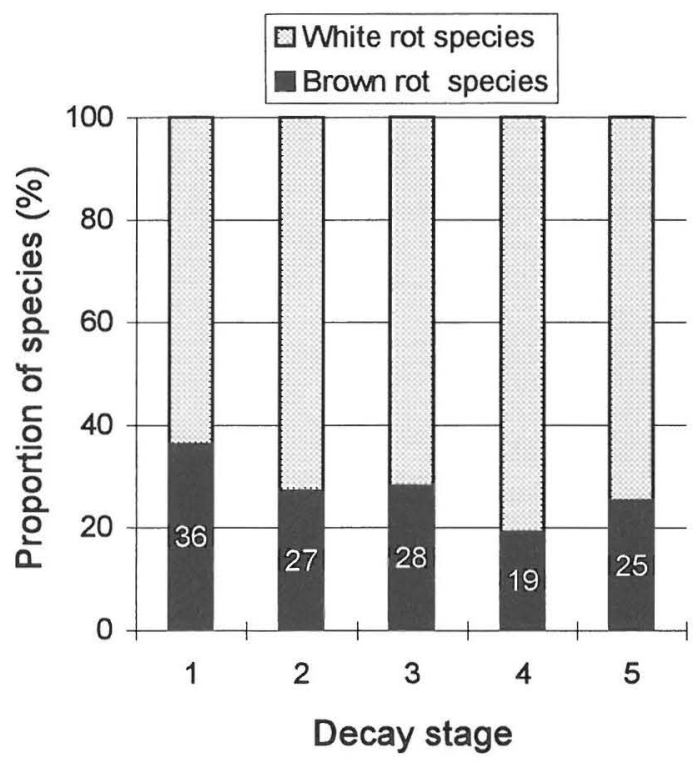

Fig. 14. Proportions of brown and white rot fungi on fallen pine (Pinus sylvestris) trunks $(\mathrm{n}=440)$ at different stages of decay (1-5).

Organization. The occurrence of fungi on pine trunks varied according to the diameter at the base of the trunks (Table 22). Amyloporia crassa, Antrodia infirma and A. primaeva were found on large trunks, while, Phanerochaete sanguinea, Piloderma croceum, Phlebiella vaga

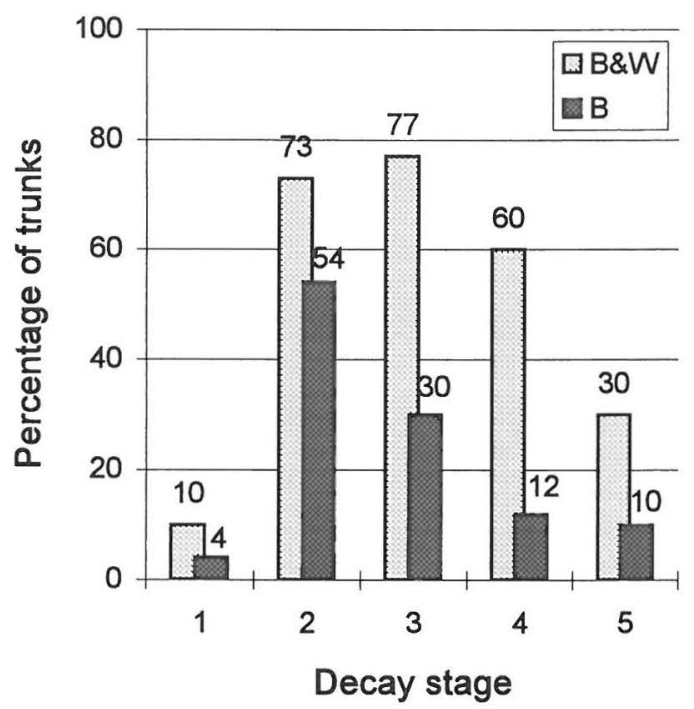

Fig. 15. Percentage of pine (Pinus sylvestris) trunks $(\mathrm{n}=$ $440)$ at different stages of decay (1-5) inhabited by brown rot fungi; $\mathrm{B}=$ percentage of trunks inhabited solely by brown rot fungi; $B \& W=$ percentage of trunks inhabited by both brown and white rot fungi.

and Chaetodermella luna mostly grew on trunks with a base diameter less than $30 \mathrm{~cm}$.

The fungi that inhabited the basal parts of the trunks were usually not found at all in the crown. On the other hand, the species that occupied the top third of the trunks were usually absent from the basal parts. Values of the Relative Locality index (R.L.), used to describe the average longitudinal distribution of fungi on the sample trunks, are summarized in Table 23. Stereum sanguinolentum (R.L. value 0.95 ) was almost totally restricted to the base. Trichaptum laricinum, Fomitopsis pinicola, Gloeophyllum protractum and Antrodia primaeva (R.L. values 0.80-0.89) also preferred the basal third of the trunks, while Panellus mitis, Chaetodermella luna, Sistotremastrum suecicum, Exidia saccharina, Resinicium furfuraceum and Phanerochaete sanguinea (R.L. values 0.42-0.58) were inhabitants of the crowns.

Successional pathways on decomposing spruce trunks

In addition to the stage of decomposition and the trunk size, the composition of the fungal community on a single spruce trunk was 
sensitive to the type of stem breakage and the history of fungal infections preceding the fall of the tree. Trunks that had broken and fallen because of butt-rot tended to harbour different mycoflora than uprooted but originally otherwise more or less healthy windfalls or trunks broken by heavy snow (Table 24).

The main species responsible for decay on spruce trees in the study area are Fomitopsis pinicola, Onnia leporina, Phellinus chrysoloma, Coniophora spp., Trichaptum laricinum, Climacocystis borealis and Stereum sanguinolentum (Eriksson 1958, Norokorpi 1979, Renvall et al. 1991b). Often these species cause extensive decay on standing and even still living trees, and then continue their work after the tree has fallen. Fomitopsis rosea was a frequent and evidently also rapid invader of fallen, undecayed spruce trunks of many kinds. By changing the chemical and physical properties of the wood, these first species influence the ability of other fungi to colonize the trunks. Species compositions thus also depend on the primary decayer(s) of the trunks, and several successional pathways were detected on decomposing spruce trunks.

Although the reasons for death of an individual tree are difficult to determine, the main agents of decay usually are easily named. All the species mentioned above occupy large volumes of wood, and the decay pattern caused by each is fairly easy to recognize. In addition, they produce large and easily identifiable basidiomes, which in most cases can be identified even years after their death. While the methods I applied do not permit a strict classification of successional pathways, seven different types were preliminarily distinguished (Table 25). The classification was made on the basis of the

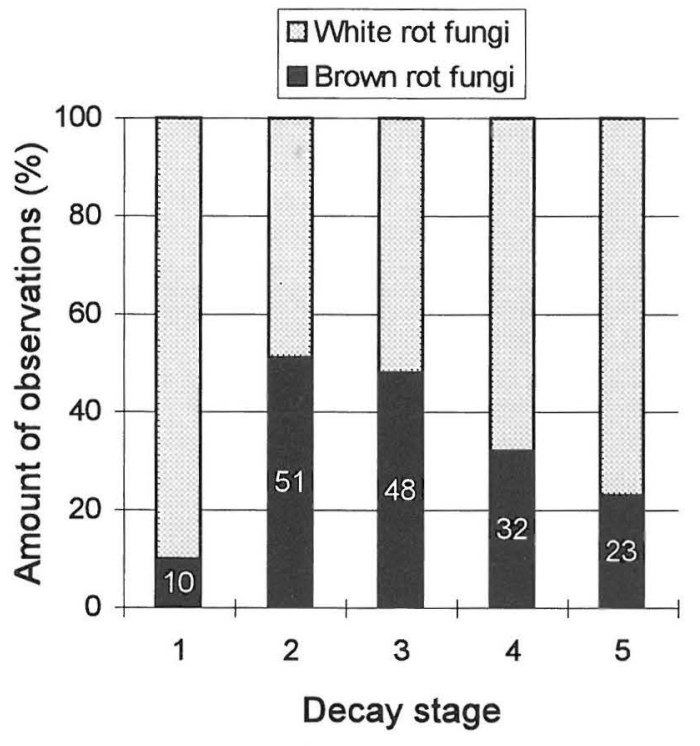

Fig. 16. Observations of brown rot fungi (in \%) on fallen pine (Pinus sylvestris) trunks $(n=440)$ at different stages of decay (1-5) as percentage of all observations.

different species compositions represented on the sample trunks and seven successional pathways, which were revealed to some extent also by the numerical treatment (TWINSPAN, DCA) of the data, are named according to the primary decayers or the type of stem breakage. The general structures of the fungal communities of the main successional pathways are outlined and compared with each other in Table 25.

Notes on the main pathways. Uprooted windfalls of spruce were almost invariably colonized by Stereum sanguinolentum, Trichaptum abietinum, Laurilia sulcata or Amylostereum chailletii. The first two species

Table 21. Values of Sørensen's Quotient of Similarity applied in comparing the species compositions of wood-inhabiting fungi on trunks of Pinus sylvestris $(\mathrm{n}=440)$ at different stages of decay $(1-5)$. Figures given in parentheses show the number of species common to the two decay stages.

\begin{tabular}{cccccc}
\hline Decay stage & 1 & 2 & 3 & 4 & 5 \\
\hline 1 & 1.000 & & & & \\
2 & $0.255(7)$ & 1.000 & & & \\
3 & $0.127(5)$ & $0.625(35)$ & 1.000 & & \\
4 & $0.055(2)$ & $0.509(27)$ & $0.615(40)$ & 1.000 & \\
5 & $0.065(1)$ & $0.406(13)$ & $0.364(16)$ & $0.464(19)$ & 1.000 \\
\hline
\end{tabular}


Table 22. Basidiomycetes found on fallen trunks of Pinus sylvestris, arranged according to the base diameter of the trunks. Species recorded less than four times have been omitted. Brown rot species are marked with *; Frb diam = diameter of the trunk at the place of basidiocarps.

\begin{tabular}{|c|c|c|c|c|c|}
\hline \multirow[t]{2}{*}{ Species } & \multicolumn{2}{|c|}{ Base diam } & \multicolumn{2}{|c|}{ Frb diam } & \multirow[t]{2}{*}{$\mathrm{n}$} \\
\hline & mean & range & mean & range & \\
\hline Amyloporia crassa* & 49 & $35-60$ & 38 & $30-45$ & 4 \\
\hline Antrodia infirma* & 48 & $40-55$ & 33 & $15-40$ & 4 \\
\hline Tricholomopsis decora & 43 & $30-60$ & 31 & $25-40$ & 6 \\
\hline Antrodia primaeva* & 42 & $15-60$ & 34 & $20-50$ & 27 \\
\hline Odonticium romellii & 41 & $20-80$ & 26 & $10-40$ & 5 \\
\hline Amylocystis lapponica* & 40 & $35-60$ & 28 & $25-30$ & 4 \\
\hline Hyphodontia breviseta & 38 & $20-80$ & 22 & $15-30$ & 8 \\
\hline Phlebia cornea & 38 & $15-60$ & 24 & $15-30$ & 9 \\
\hline Stereum sanguinolentum & 38 & $15-90$ & 32 & $15-80$ & 42 \\
\hline Sistotremastrum suecicum & 37 & $25-50$ & 20 & $5-35$ & 17 \\
\hline Athelia decipiens & 36 & $20-60$ & 21 & $15-25$ & 4 \\
\hline Coniophora olivacea* & 36 & $25-60$ & 24 & $15-35$ & 7 \\
\hline Fomitopsis pinicola & 36 & $10-80$ & 33 & $15-80$ & 11 \\
\hline Phellinus nigrolimitatus & 36 & $25-45$ & 26 & $15-40$ & 8 \\
\hline Phellinus viticola & 36 & $10-80$ & 26 & $10-60$ & 46 \\
\hline Tubulicrinis calothrix & 36 & $15-70$ & 24 & $5-40$ & 4 \\
\hline Aleurodiscus lividocoeruleus & 34 & $15-45$ & 23 & $10-40$ & 4 \\
\hline Ceraceomyces borealis & 34 & $25-40$ & 24 & $10-40$ & 8 \\
\hline Oligoporus sericeomollis & 34 & $15-80$ & 23 & $10-40$ & 24 \\
\hline Resinicium furfuraceum & 34 & $15-50$ & 18 & $10-35$ & 18 \\
\hline Antrodia albobrunnea: & 33 & $15-60$ & 22 & $5-50$ & 73 \\
\hline Botryobasidium subcoronatum & 33 & $10-60$ & 20 & $10-50$ & 30 \\
\hline Globulicium hiemale & 33 & $10-50$ & 26 & $10-45$ & 12 \\
\hline Hyphoderma setigerum & 33 & $20-60$ & 24 & $10-50$ & 18 \\
\hline Panellus mitis & 33 & $20-40$ & 13 & $10-15$ & 4 \\
\hline Phlebiopsis gigantea & 33 & $20-60$ & 23 & $15-30$ & 11 \\
\hline Skeletocutis lenis & 33 & $10-60$ & 23 & $5-50$ & 43 \\
\hline Amyloporia xantha* & 32 & $10-80$ & 23 & $5-50$ & 129 \\
\hline Gloeophyllum protractum & 32 & $10-70$ & 26 & $10-40$ & 14 \\
\hline Trechispora farinacea & 32 & $10-60$ & 19 & $5-40$ & 25 \\
\hline Trichaptum fuscoviolaceum & 32 & $15-60$ & 25 & $10-50$ & 32 \\
\hline Hyphoderma praetermissum & 31 & $20-50$ & 23 & $10-35$ & 19 \\
\hline Hypochnicium albostramineum & 31 & $15-40$ & 18 & $5-35$ & 4 \\
\hline Junghuhnia luteoalba & 31 & $15-40$ & 23 & $15-35$ & 4 \\
\hline Leucogyrophana romellii* & 31 & $15-50$ & 19 & $10-40$ & 24 \\
\hline Postia lateritia* & 31 & $10-55$ & 23 & $10-40$ & 32 \\
\hline Trichaptum laricinum & 31 & $15-30$ & 24 & $10-40$ & 6 \\
\hline Exidia saccharina & 30 & $15-60$ & 17 & $10-30$ & 15 \\
\hline Postia hibernica* & 30 & $10-60$ & 19 & $5-45$ & 34 \\
\hline Trichaptum abietinum & 30 & $15-50$ & 20 & $10-35$ & 13 \\
\hline Antrodia sinuosa* & 29 & $10-50$ & 22 & $10-40$ & 31 \\
\hline Botryobasidium botryosum & 29 & $15-60$ & 22 & $15-45$ & 32 \\
\hline Ceraceomyces sublaevis & 29 & $10-40$ & 21 & $10-40$ & 9 \\
\hline Chaetodermella luna* & 29 & $10-60$ & 16 & $5-45$ & 36 \\
\hline Tubulicrinis medius & 28 & $20-40$ & 19 & $10-35$ & 6 \\
\hline Gloeophyllum sepiarium* & 29 & $25-35$ & 21 & $10-30$ & 5 \\
\hline Phlebiella vaga & 28 & $15-60$ & 19 & $5-50$ & 35 \\
\hline Antrodiella parasitica & 26 & $10-40$ & 26 & $10-40$ & 8 \\
\hline Piloderma croceum & 26 & $10-70$ & 18 & $5-40$ & 77 \\
\hline Sistotrema muscicola & 26 & $15-50$ & 22 & $15-55$ & 8 \\
\hline Phlebia segregata & 24 & $15-35$ & 17 & $15-20$ & 4 \\
\hline Phanerochaete sanguinea & 23 & $10-45$ & 14 & $5-25$ & 10 \\
\hline Piloderma olivaceum & 21 & $10-30$ & 12 & $5-20$ & 6 \\
\hline
\end{tabular}


Table 23. Values of the Relative Locality index (R.L.) applied in describing and comparing the average proximal vs. distal location of the wood-inhabiting fungi on fallen trunks of Pinus sylvestris $(n=440)$. Brown rot species are marked with *; $\mathrm{n}=$ number of observations.

\begin{tabular}{|c|c|c|c|c|c|}
\hline Species & R.L. & $\mathrm{n}$ & Species & R.L. & $\mathrm{n}$ \\
\hline Stereum sanguinolentum & 0.95 & 42 & Phellinus nigrolimitatus & 0.70 & 8 \\
\hline Sistotrema muscicola & 0.89 & 7 & Hyphodontia breviseta & 0.70 & 8 \\
\hline Trichaptum laricinum & 0.88 & 5 & & 0.69 & 17 \\
\hline Gloeophyllum protractum* & 0.82 & 9 & $\begin{array}{l}\text { Antrodia sinuosa } \\
\text { Coniophora olivacea* }\end{array}$ & 0.69 & 7 \\
\hline Fomitopsis pinicola & 0.82 & 10 & Hyphoderma setigerum & 0.68 & 17 \\
\hline Antrodia primaeva* & 0.80 & 23 & Postia hibernica* & 0.67 & 27 \\
\hline Phlebia segregata & 0.80 & 4 & Ceraceomyces borealis & 0.67 & 7 \\
\hline Odonticium romellii & 0.78 & 5 & Trichaptum abietinum & 0.67 & 9 \\
\hline Amyloporia crassa* & 0.78 & 4 & Phellinus viticola & 0.66 & 30 \\
\hline Antrodia infirma* & 0.78 & 4 & Phlebiella vaga & 0.66 & 28 \\
\hline Antrodiella parasitica & 0.78 & 4 & Piloderma croceum & 0.65 & 64 \\
\hline Trichaptum fuscoviolaceum & 0.77 & 34 & Antrodia albobrunnea* & 0.63 & 52 \\
\hline Junghuhnia luteoalba & 0.75 & 4 & Athelia decipiens & 0.63 & 4 \\
\hline Botryobasidium botryosum & 0.74 & 29 & Leucogyrophana romellii* & 0.62 & 23 \\
\hline Postia lateritia & 0.74 & 26 & Botryobasidium subcoronatum & 0.61 & 22 \\
\hline Oligoporus sericeomollis\% & 0.73 & 19 & Trechispora farinacea & 0.60 & 24 \\
\hline Ceraceomyces sublaevis & 0.73 & 8 & & 058 & 6 \\
\hline Gloeophyllum sepiarium* & 0.73 & 4 & Phanerochaete sanguinea & $\begin{array}{l}0.58 \\
0.58\end{array}$ & 6 \\
\hline Globulicium hiemale & 0.72 & 10 & Tubulicrinis medius & 0.58 & 6 \\
\hline Tricholomopsis decora & 0.72 & 5 & Phlebia cornea & 0.58 & 5 \\
\hline Phlebiopsis gigantea & 0.72 & 11 & Resinicium furfuraceum & 0.56 & 17 \\
\hline Amyloporia xantha: & 0.72 & 72 & Exidia saccharina & 0.55 & 13 \\
\hline Skeletocutis lenis & 0.71 & 35 & Sistotremastrum suecicum & 0.55 & 11 \\
\hline Tubulicrinis calothrix & 0.71 & $\begin{array}{r}35 \\
4\end{array}$ & Chaetodermella luna* & 0.52 & 30 \\
\hline Hyphoderma praetermissum & 0.71 & $\begin{array}{r}4 \\
15\end{array}$ & Panellus mitis & 0.42 & 4 \\
\hline
\end{tabular}

were seldom found on trunks which had been primarily decayed by other fungi. Although 63 uprooted trunks were checked, many abundant saprotrophs of spruce, e.g., Phellinus nigrolimitatus, P. ferrugineofuscus, Skeletocutis odora and Leptoporus mollis, were not found at all.

The trunks occupied by Fomitopsis rosea maintained a characteristic and fairly uniform composition of fungi. $F$. rosea was very often associated with Phlebia centrifuga, Phellinus ferrugineofuscus, Skeletocutis odora and, in particular, Amylocystis lapponica. At later stages of decay it frequently co-occurred with Phellinus nigrolimitatus, which, together with Amylocystis lapponica, was the dominant fungus on trunks at the decay stages 3 and 4 . Another characteristic feature for trunks in advanced decay was the large number of species. However, Trichaptum abietinum and $T$. laricinum were then totally absent.

Trunks displaying the successional pathway opened by Onnia leporina were characterized by high species diversity and a clear crown vs. butt distribution of fungi. O. leporina was restricted to basal parts, while Trichaptum abietinum, the most frequent saprotroph on these trunks, was found only on decayed crowns, mostly together with Gloeophyllum sepiarium or Antrodia serialis. Middle parts of the trunks were often inhabited by Phellinus ferrugineofuscus, Fomitopsis rosea and Phlebia centrifuga. Phellinus nigrolimitatus was recorded only four times on trunks that were primarily decayed by Onnia leporina. 
Phellinus chrysoloma is a strong invader, which occupies large volumes of the trunks. The compound basidiocarps it produces may be as much as several metres long. Accordingly, trunks primarily decayed by this polypore present a distinct successional pathway. Characteristic features are the small number of species and evidently a very rapid decomposition of wood. Often wood was considerably softened and undergoing a strong process of decomposition even when the bark was virtually intact and almost completely covered the trunk. Moreover, epiphytic lichens and bryohytes were few, making up only small fragmentary patches. P. chrysoloma cooccurred four times with Stereum sanguinolentum. Typical adjoining saprotrophs were Trichaptum abietinum and $T$. fuscoviolaceum and at later stages of decay also Phellinus nigrolimitatus. The almost totally decomposed trunks (D.S. 5) were twice found to be inhabited by Skeletocutis stellae.

Table 24. Occurrence of selected Basidiomycetes on fallen trunks of Picea abies subsp. obovata, arranged according to the type of stem breakage. Brown rot species are marked with *. Types of stem breakage: $\operatorname{stump}=$ stump+trunk; upr $=$ uprooted trunk with root plate; root $=$ trunk fallen because of rot in main roots; snow = snow break; $n=$ number of observations.

\begin{tabular}{|c|c|c|c|c|c|}
\hline \multirow[t]{2}{*}{ Species } & \multicolumn{4}{|c|}{ Breakage type } & \multirow[t]{2}{*}{$\mathrm{n}$} \\
\hline & stump & upr & root & snow & \\
\hline Skeletocutis stellae & 100 & - & - & - & 5 \\
\hline Trichaptum laricinum & 100 & - & - & - & 9 \\
\hline Phellinus nigrolimitatus & 83 & - & 15 & 2 & 55 \\
\hline Phlebia centrifuga & 83 & 2 & 15 & - & 46 \\
\hline Antrodia serialis & 74 & 7 & 15 & 4 & 27 \\
\hline Skeletocutis odora & 74 & - & 26 & - & 19 \\
\hline Climacocystis borealis & 71 & 14 & 14 & - & 7 \\
\hline Phellinus chrysoloma & 71 & 6 & 16 & 6 & 31 \\
\hline P. ferrugineofuscus & 71 & - & 23 & 6 & 31 \\
\hline Onnia leporina & 69 & 9 & 13 & 9 & 29 \\
\hline Coniophora arida* & 68 & 22 & - & - & 9 \\
\hline Antrodiella parasitica & 67 & 17 & 17 & - & 12 \\
\hline Gloeoporus taxicola & 67 & 8 & 17 & 8 & 12 \\
\hline Peniophora pithya & 67 & - & 17 & 17 & 6 \\
\hline Fomitopsis rosea* & 66 & 6 & 27 & 1 & 89 \\
\hline Phellinus viticola & 64 & 9 & 27 & - & 11 \\
\hline Phlebiella vaga & 64 & 7 & 21 & 7 & 14 \\
\hline Columnocystis abietina* & 63 & 11 & 26 & - & 27 \\
\hline Amylocystis lapponica* & 62 & 2 & 36 & - & 42 \\
\hline Dichostereum granulosum & 60 & - & 40 & - & 5 \\
\hline Hyphodontia breviseta & 60 & 7 & 7 & 27 & 15 \\
\hline Fomitopsis pinicola* & 59 & 18 & 18 & 5 & 39 \\
\hline Tubulicrinis calothrix & 59 & 17 & 25 & - & 12 \\
\hline Trichaptum abietinum & 56 & 23 & 12 & 10 & 52 \\
\hline T. fuscoviolaceum & 55 & 9 & 27 & 9 & 11 \\
\hline Asterodon ferruginosus & 50 & 17 & 34 & - & 6 \\
\hline Hyphoderma argillaceum & 50 & 38 & 12 & - & 8 \\
\hline Gloeophyllum sepiarium* & 49 & 23 & 24 & 3 & 34 \\
\hline Leptoporus mollis* & 44 & - & 44 & 11 & 9 \\
\hline Laurilia sulcata & 43 & 30 & 26 & - & 23 \\
\hline Coniophora olivacea* & 37 & 33 & 23 & 7 & 27 \\
\hline Amylostereum chailletii & 18 & 36 & 27 & 18 & 11 \\
\hline Stereum sanguinolentum & 17 & 75 & - & 8 & 41 \\
\hline Exidia saccharina & 14 & 71 & - & 14 & 7 \\
\hline Peniophora septentrionalis & - & 20 & - & 80 & 5 \\
\hline
\end{tabular}


Fomitopsis pinicola causes extensive brown rot in basal parts of the spruce trunks. Often it cooccurred with $F$. rosea, Phlebia centrifuga and Phellinus ferrugineofuscus, which, however, were mostly found in the middle of the trunks. Trichaptum abietinum was the most abundant fungus in the top third of the trunks. Fomitopsis pinicola was occasionally associated with other aggressive decomposers, e.g., Phellinus chrysoloma and Laurilia sulcata. Although 11 trunks primarily decayed by Fomitopsis pinicola at decay stages 3 and 4 were carefully studied, Phellinus nigrolimitatus was recorded only once.

Trichaptum laricinum is an aggressive decomposer of trunks of medium or smaller size (base diameter $10-40 \mathrm{~cm}$ ), especially in stunted spruce stands bordering swampy areas. The trunks already were considerably softened (D.S. 3-4) and extensively decayed when they fell. Being a rapid invader, which occupies most of the basal third of the trunks, $T$. laricinum grew mostly alone. The commonest associates were Fomitopsis rosea and Gloeophyllum sepiarium.

The number of species per trunk (Table 25) was largest on trunks primarily decayed by a particular brown-rot fungus (not Fomitopsis spp.), evidently Coniophora sp., and which had fallen because of the decay. Very often the main roots were broken as a result of the dry cubical brown rot, or the trunk had fallen because of extensive butt-rot. Although the primary decayers of these trunks while they were standing are unknown, and may even include several species, the sample trunks that exhibited the above-described pattern are here included under a single pathway of succession, named after the genus Coniophora. Because only 12 trunks were studied, very little can be said about the species composition. The most frequent fungi fruiting on the trunks were Coniophora olivacea, Laurilia sulcata and Fomitopsis rosea.

Successional pathways on decomposing pine trunks

Most of the pine trunks studied were either uprooted by strong wind while alive, or, more often, had fallen decades after their death, as decorticated, kelo trees that had dried while standing. The compositions of fungal com-

Table 25. Outlines of the main successional pathways on decomposing trunks of Picea abies subsp. obovata.

Pathway Core species

1. Uprooted trunks

2. Fomitopsis rosea

3. Onnia leporina

4. Phellinus chrysoloma

5. Fomitopsis pinicola

6. Trichaptum laricinum

7. Coniophora
Stereum sanguinolentum, Trichaptum abietinum, Amylostereum chailletii, Laurilia sulcata

Fomitopsis rosea, Amylocystis lapponica, Phlebia centrifuga, Phellinus ferrugineofuscus, Skeletocutis odora, Phellinus nigrolimitatus Onnia leporina, Trichaptum abietinum, Phellinus ferrugineofuscus, Gloeophyllum sepiarium

Phellinus chrysoloma, Trichaptum abietinum, Phellinus nigrolimitatus Fomitopsis pinicola, F. rosea, Phlebia centrifuga, Phellinus ferrugineofuscus, Trichaptum abietinum

Trichaptum laricinum

Coniophora olivacea, C. arida, Laurilia sulcata, Fomitopsis rosea

\begin{tabular}{|c|c|c|c|c|c|c|c|}
\hline & \multicolumn{7}{|c|}{ Successional pathway } \\
\hline & 1 & 2 & 3 & 4 & 5 & 6 & 7 \\
\hline Number of trunks studied & 63 & 48 & 27 & 25 & 23 & 13 & 12 \\
\hline Number of species & 43 & 52 & 47 & 34 & 28 & 18 & 37 \\
\hline Number of brown rot species & 9 & 9 & 11 & 6 & 7 & 5 & 11 \\
\hline Number of observations & 135 & 214 & 137 & 64 & 73 & 25 & 72 \\
\hline Amount $(\%)$ of observations/brown rot fungi & 24 & 52 & 26 & 23 & 64 & 44 & 28 \\
\hline Species number/trunk (mean \pm S.D.) & $1.7 \pm 1.3$ & $4.5 \pm 2.4$ & $4.2 \pm 2.5$ & $2.7 \pm 1.6$ & $3.7 \pm 1.6$ & $2.8 \pm 1.8$ & $4.8 \pm 3.3$ \\
\hline Species number/decay stage 3 (mean \pm S.D.) & $3.4 \pm 2.5$ & $4.7 \pm 2.9$ & $4.8 \pm 2.2$ & $2.4 \pm 1.5$ & $4.5 \pm 1.9$ & $2.4 \pm 0.5$ & $8.0 \pm 3.6$ \\
\hline
\end{tabular}


munities on these two types of trunks differed greatly from each other and represent two main pathways of succession (Table 27). Trunks heavily damaged by fire mostly hosted still other fungi and form a third major pathway (Table 13). As shown in Table 26, the compositions of fungi depended on the type of stem breakage. Although only a few trunks (11) had fallen as the obvious result of extensive butt decay, the species compositions were unique, allowing two futher pathways to be distinguished: one in which decay was attributable to Fomitopsis pinicola and another in which it was due to Trichaptum laricinum. Trunks that were primarily colonized by Fomitopsis pinicola were also inhabited by Trichaptum abietinum. The five pathways of decomposition in pine trunks are outlined in Table 27.

Fresh windfalls were colonized by Stereum sanguinolentum, Trichaptum fuscoviolaceum, Hyphoderma setigerum, Phlebiopsis gigantea and Exidia saccharina, whereas kelo trees totally lacked these species and were predominantly colonized by Amyloporia xantha instead. A. xantha was often found alone, without associates. However, many other species, e.g., Phellinus viticola, Postia lateritia, P. hibernica, Antrodia primaeva, Oligoporus sericeomollis, Antrodia sinuosa, Gloeophyllum protractum and the late-stagedominants Antrodia albobrunnea and Skeletocutis lenis, occurred frequently on these trunks. Many times these species were fruiting alone, but almost all of them sometimes co-occurred with each other as well. Because the origins of the trunks at decay stage 4 and 5 were difficult to determine, the characteristics and the species compositions of the successional pathways on decomposing pine trunks were not readily apparent. A subdivision of the successional pathways on decorticated kelo trees was difficult to make therefore. TWINSPAN (Fig. 22) nevertheless divided the fallen, uncharred snags (kelo trees) into three groups, (subpathways 2a, 2b, 2c) on the basis of their main decayer: Amyloporia xantha, Antrodia primaeva or Antrodia albobrunnea.

\section{Ordination}

The DCA ordinations of the sample trunks and species revealed a clear successional grouping of the data. Both spruce and, in particular, pine trunks and the fungi on them were screened into fairly strong patterns of succession (Figs. 17-20). The first ordination axis in all the ordinations was strongly correlated with the decay stage, thus corresponding with the time and succession gradient. Accordingly, sample trunks at decay stage 1 and species that favouring early stages of decomposition had higher values on this axis and were plotted into the upper parts of diagrams. The first axis was also (negatively) correlated with the amount of bark on sample trunks. Because of small differences in the species compositions on sample trunks under each decay stage, the number of overprinted trunks was high in all the ordinations.

The ecological interpretation of the second ordination axis was not readily apparent. The axis did not separate either site types or thick trunks from thin ones. In the ordination of spruce trunks (Figs. 17 and 18) it seemed to be related to the separation of different successional pathways on decomposing trunks, sorting out the primary decayers (Climacocystis borealis, Peniophora septentrionalis, Phellinus chrysoloma, Stereum sanguinolentum, Fomitopsis rosea, Onnia leporina, Fomitopsis pinicola, Laurilia sulcata) and the trunks occupied by these fungi - that strongly influenced the subsequent species compositions (Table 25). These primary fungi occupy large volumes of wood and evidently are strong competitors in the primary resource capture. In the ordination of pine trunks, the second axis was related to differences in the species composition between the uprooted windfalls, decorticated kelo trees and charred trunks. The strongly charred trunks and the species that occurred frequently on charred wood obtained higher values on this axis and are concentrated to the right in the diagrams (Figs. 19 and 20). The third and fourth axes (not shown; eigenvalues: Picea 0.545 and 0.461 ; Pinus 0.639 and 0.557 ) did not reveal any additional ecologically meaningful patterns in the data of either spruce or pine trunks.

The DCA ordinations also indicated that there was considerable overlap in the species compositions on trunks at different stages of decay. On spruce the decay stages 3 and 4 and on pine the decay stages 2 and 3 , in particular, harboured many species in common. Thus the sample trunk grouping, which was based on the presence and absence of fungi only, did not always reveal ecological differences between the stages of decay. 
Table 26. Occurrence of selected species of wood-rotting Basidiomycetes on fallen trunks of Pinus sylvestris $(n=440)$, arranged according to type of stem breakage. Brown rot species are marked with *. Types of stem breakage: upr $=$ uprooted trunk with a root plate; stump = stump+trunk; $n=$ number of observations.

\begin{tabular}{|c|c|c|c|c|c|c|c|}
\hline \multirow{2}{*}{$\begin{array}{l}\text { Species } \\
\text { Ceraceomyces borealis }\end{array}$} & \multicolumn{2}{|c|}{$\begin{array}{l}\text { Breakage type } \\
\text { upr stump }\end{array}$} & \multirow{2}{*}{$\begin{array}{l}\mathrm{n} \\
\mathrm{7} \\
\end{array}$} & \multirow{2}{*}{$\begin{array}{l}\text { Species } \\
\text { Antrodia albobrunnea" }\end{array}$} & \multicolumn{2}{|c|}{$\begin{array}{l}\text { Breakage type } \\
\text { upr stump }\end{array}$} & $\mathrm{n}$ \\
\hline & 100 & - & & & 67 & 33 & 48 \\
\hline Hyphoderma setigerum & 100 & - & 18 & Gloeophyllum protractum* & 67 & 33 & 9 \\
\hline Odonticium romellii & 100 & - & 5 & Phlebia cornea & 67 & 33 & 6 \\
\hline Phlebiopsis gigantea & 100 & - & 11 & Phellinus viticola & 61 & 39 & 28 \\
\hline Sistotrema muscicola & 100 & - & 7 & Postia hibernica* & 60 & 40 & 25 \\
\hline Stereum sanguinolentum & 95 & 5 & 41 & P. lateritia* & 58 & 42 & 24 \\
\hline Trichaptum fuscoviolaceum & 94 & 6 & 33 & Antrodia sinuosa* & 56 & 44 & 16 \\
\hline Exidia saccharina & 91 & 11 & 11 & Amyloporia xantha* & 55 & 45 & 67 \\
\hline Phlebiella vaga & 89 & 11 & 27 & Trechispora farinacea & 54 & 46 & 24 \\
\hline Hyphoderma praetermissum & 86 & 14 & 14 & Chaetodermella luna* & 52 & 48 & 31 \\
\hline Hyphodontia breviseta & 83 & 17 & 6 & Tricholomopsis decora & 50 & 50 & 6 \\
\hline Skeletocutis lenis & 83 & 17 & 35 & Ceraceomyces sublaevis & 50 & 50 & 8 \\
\hline Antrodia primaeva* & 79 & 21 & 24 & Phellinus nigrolimitatus & 50 & 50 & 8 \\
\hline Leucogyrophana romellii & 78 & 22 & 23 & Phanerochaete sanguinea & 33 & 67 & 6 \\
\hline Oligoporus sericeomollis* & 78 & 22 & 18 & Fomitopsis pinicola* & 33 & 67 & 9 \\
\hline Sistotremastrum suecicum & 73 & 27 & 11 & Globulicium hiemale & 25 & 75 & 8 \\
\hline Coniophora olivacea* & 71 & 29 & 7 & Trichaptum abietinum & 14 & 86 & 7 \\
\hline Resinicium furfuraceum & 71 & 29 & 17 & T. laricinum & - & 100 & 5 \\
\hline
\end{tabular}

Table 27. Main successional pathways on decomposing trunks of Pinus sylvestris.

\begin{tabular}{ll}
\hline Pathway & Core species \\
\hline 1. Uprooted trunks & $\begin{array}{l}\text { Stereum sanguinolentum, Trichaptum fuscoviolaceum, Hyphoderma } \\
\text { setigerum, Phlebiopsis gigantea }\end{array}$ \\
2. Decorticated trunks & Amyloporia xantha, Chaetodernella luna, Phellinus viticola, Postia \\
(kelo trees, dried while standing) & lateritia, P. hibernica, Antrodia albobrunnea, Skeletocutis lenis, Oligoporus \\
& sericeomollis, Antrodia sinuosa, Gloeophyllum protractum \\
3. Charred trunks & Piloderma croceum, Antrodia primaeva, Leucogyrophana romellii, \\
& Ceraceomyces borealis \\
4. Fomitopsis pinicola & Fomitopsis pinicola, Trichaptum abietinum \\
5. Trichaptum laricinum & Trichaptum laricinum \\
\hline
\end{tabular}

\section{TWINSPAN classification}

The TWINSPAN classifications of the sample trunk data, which were performed on the basis of the presence or absence of fungal species, supported the general successional patterns of the material and helped in outlining the main pathways of succession on decomposing trunks. However, they did not reveal any additional ecological groupings of the species or trunks that 
Axis 1

EIG. 0.858

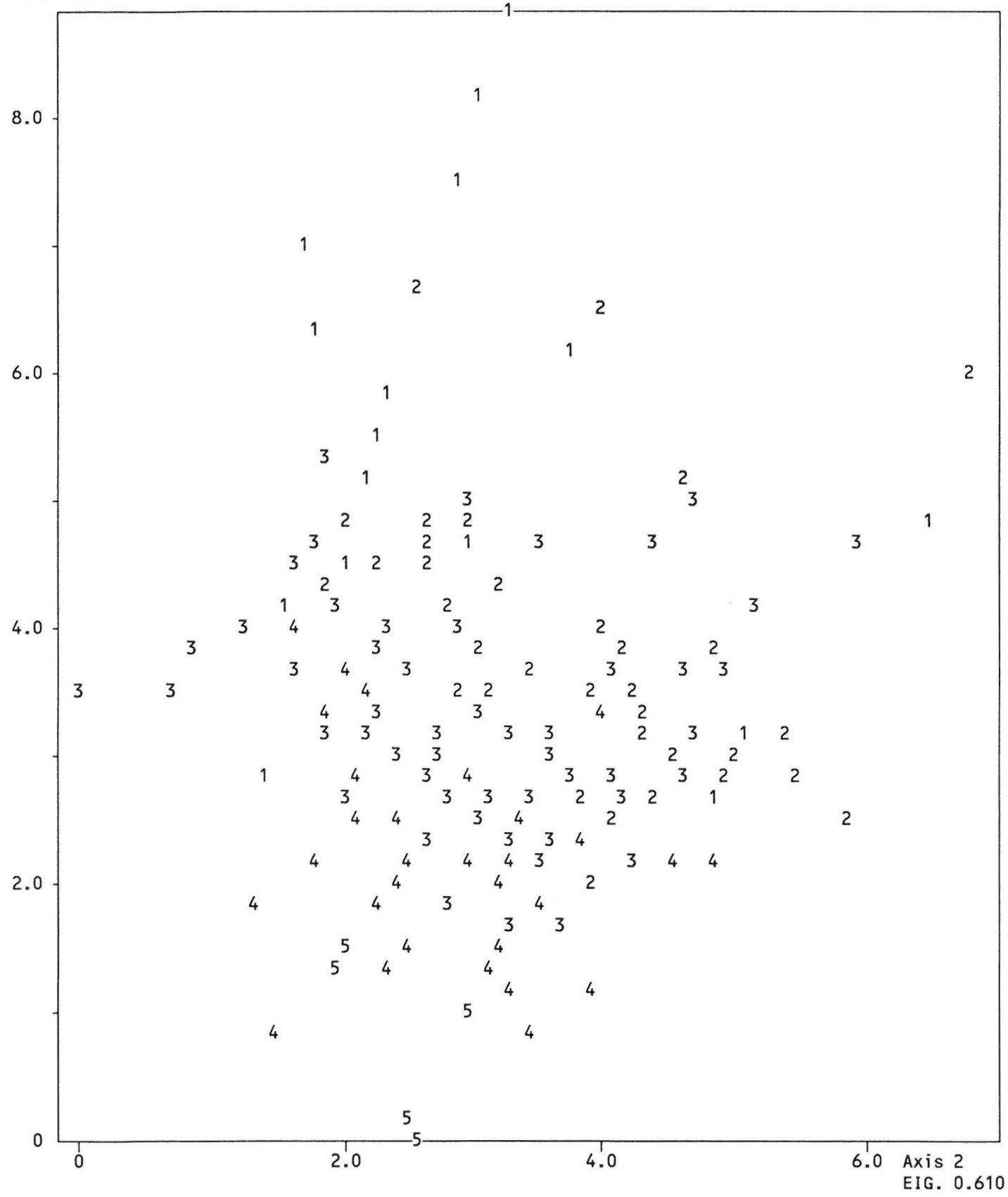

Fig. 17. DCA ordination (the first two axes) of fallen, decomposing spruce (Picea abies subsp. obovata) trunks on the basis of the composition of wood-inhabiting Basidiomycetes (presence/absence). Numbers refer to sample trunks at different stages (1-5) of decay. Species occurring on fewer than three sample trunks have been excluded from the ordination. 
Axis 1

EIG.0.858

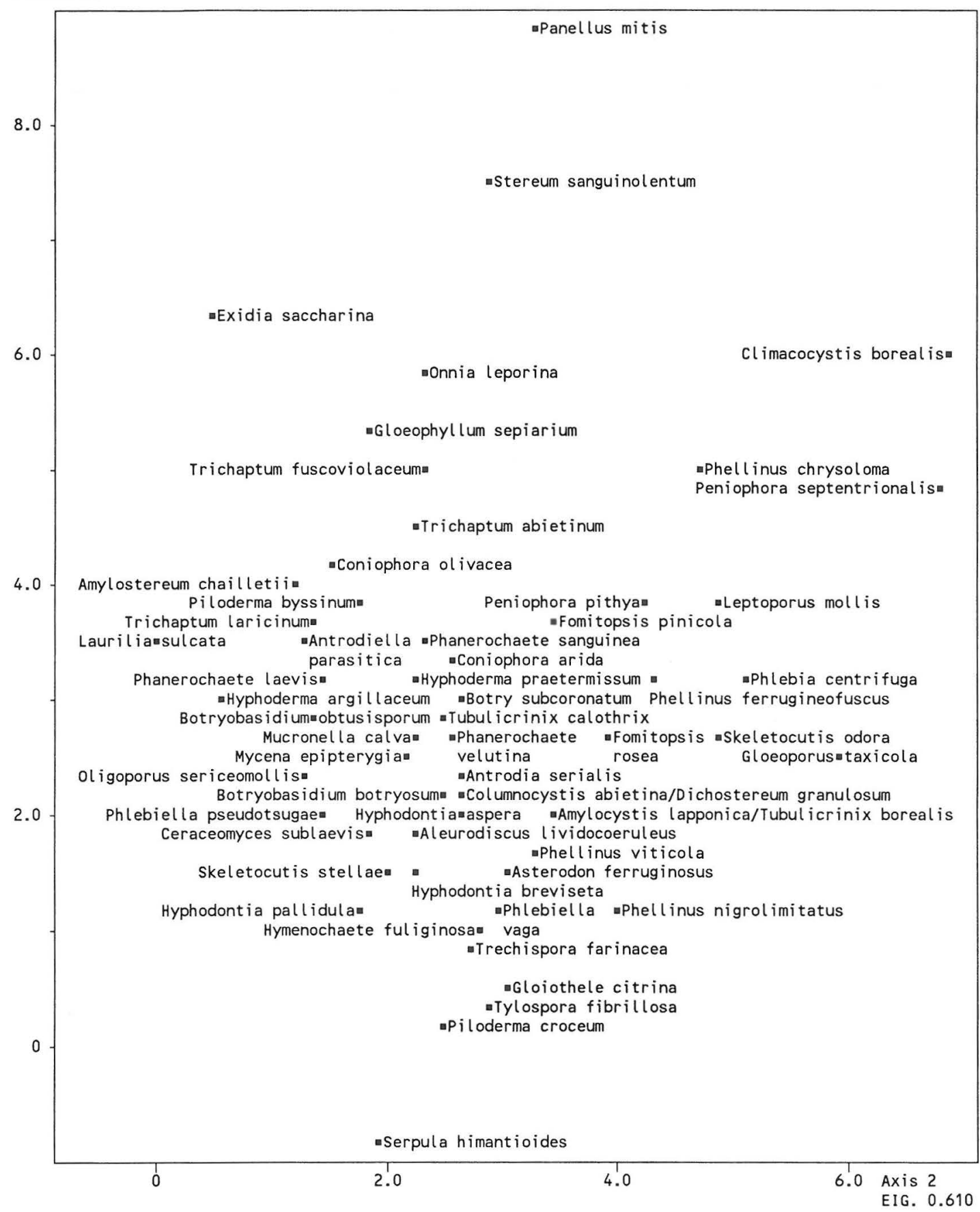

Fig. 18. DCA-ordination of Basidiomycetes (presence/absence) on fallen, decomposing trunks of Picea abies subsp. obovata. Species occurring on fewer than three sample trunks have been excluded from the ordination. 
could significantly help the ecological interpretation of the data.

In the classification of the spruce trunks (Fig. 21 ), freshly fallen, corticated and uprooted windfalls (mostly D.S. 1) emerged on the first divisional level from the rest of the material (trunks at decay stages 2-5). The indicator species of this group was Stereum sanguinolentum. On the second divisional level, where these two main groups of sample trunks were divided into four subgroups, the indicator species for the trunks at the decay stages 2-5 were Coniophora olivacea, Gloeophyllum sepiarium, Laurilia sulcata, Phellinus chrysoloma and Trichaptum abietinum. The trunks characterized by these fungi were separated from the trunks inhabited by Amylocystis lapponica, Fomitopsis rosea, Phellinus nigrolimitatus and Phlebia centrifuga. Fresh trunks (D.S. 1) were divided into two subgroups according to the presence or absence of Onnia leporina (Fig. 21, group 7)

The third divisional level classified the data into six further groups. The late successional trunks (D.S. 3-5) characterized by Piloderma croceum, Hyphodontia breviseta, Tylospora fibrillosa and, in particular, Phellinus nigrolimitatus (Fig. 21, group 2), were separated from the trunks characterized by Fomitopsis rosea, Amylocystis lapponica, Phellinus ferrugineofuscus and Phlebia centrifuga (D.S. $2-3$, group 1). Trunks which were decayed by either Climacocystis borealis (group 3) or Coniophora olivacea, Gloeophyllum sepiarium and Laurilia sulcata (group 4) were divided as well. Of the freshly fallen trunks (dominated by Stereum sanguinolentum), the third divisional level further divided the trunks into those characterised either by Exidia saccharina (and Stereum sanguinolentum group 5) or Panellus mitis (group 6).

As in the spruce trunk data, the first divisional level in the TWINSPAN analysis of the pine trunks (Fig. 22) separated the corticated and uprooted windfalls (mostly D.S. 1) from the rest of the trunks (decorticated, D.S. 2-5). Trichaptum fuscoviolaceum, Stereum sanguinolentum and Hyphoderma setigerum were the indicator species of the windfall group. The second level divisions divided the corticated trunks into two groups according to the presence or absence of Antrodiella parasitica, Hypochnicium albostramineum and Trichaptum abietinum (Fig. 22, group 7), while the decorticated trunks (mostly kelo trees) were divided into two groups characterized by the presence of either Antrodia albobrunnea and Piloderma croceum (mostly D.S. 3-4), or Amyloporia xantha and Antrodia primaeva (D.S. 2-3). The latter group consisted of trunks that almost exclusively hosted A. primaeva, Trichaptum laricinum or Amyloporia xantha.

At the third level, three of the second level groups were further divided into two subgroups each. The first two of the groups resulting were either dominated by Antrodia primaeva (Fig. 22, group 1) or Amyloporia xantha (or Trichaptum laricinum) (group 2). The other second level group consisting of decorticated trunks, inhabited by Antrodia albobrunnea and Piloderma croceum (and Postia lateritia and Phellinus viticola), was divided on the third level according to the presence or absence of Ceraceomyces borealis. Accordingly, the strongly charred trunks that hosted $C$. borealis emerged as a clear-cut ecological unit (group 3). The indicator species of the other subgroup (group 4) was Antrodia albobrunnea. The freshly fallen windfalls characterized by the presence of Trichaptum fuscoviolaceum, Stereum sanguinolentum and Hyphoderma setigerum were on the third level divided into subgroups with indicator species either Trichaptum fuscoviolaceum and Hyphoderma setigerum (group 5) or Phlebiopsis gigantea and Amyloporia xantha (group 6).

\section{Discussion}

Species composition and organization

Differences in the species compositions between the forest site types can perhaps best be explained by the microclimatical characteristics of the sites. However, the differences are evidently also due to the size variation of the trunks between the site types. This was seen, for example, in Climacocystis borealis and Laurilia sulcata, which clearly preferred brookside forests, but even there were almost exclusively restricted to the basal parts of the thickest trunks.

White rot fungi were found to dominate at the beginning of the decomposition, as well as on almost totally decomposed trunks. Most white rot species were fairly clearly restricted to a certain stage of decay, while many brown rot fungi stood out as dominant species during several successiove stages of decay. Many strong 
Axis 1

EIG. 0.964

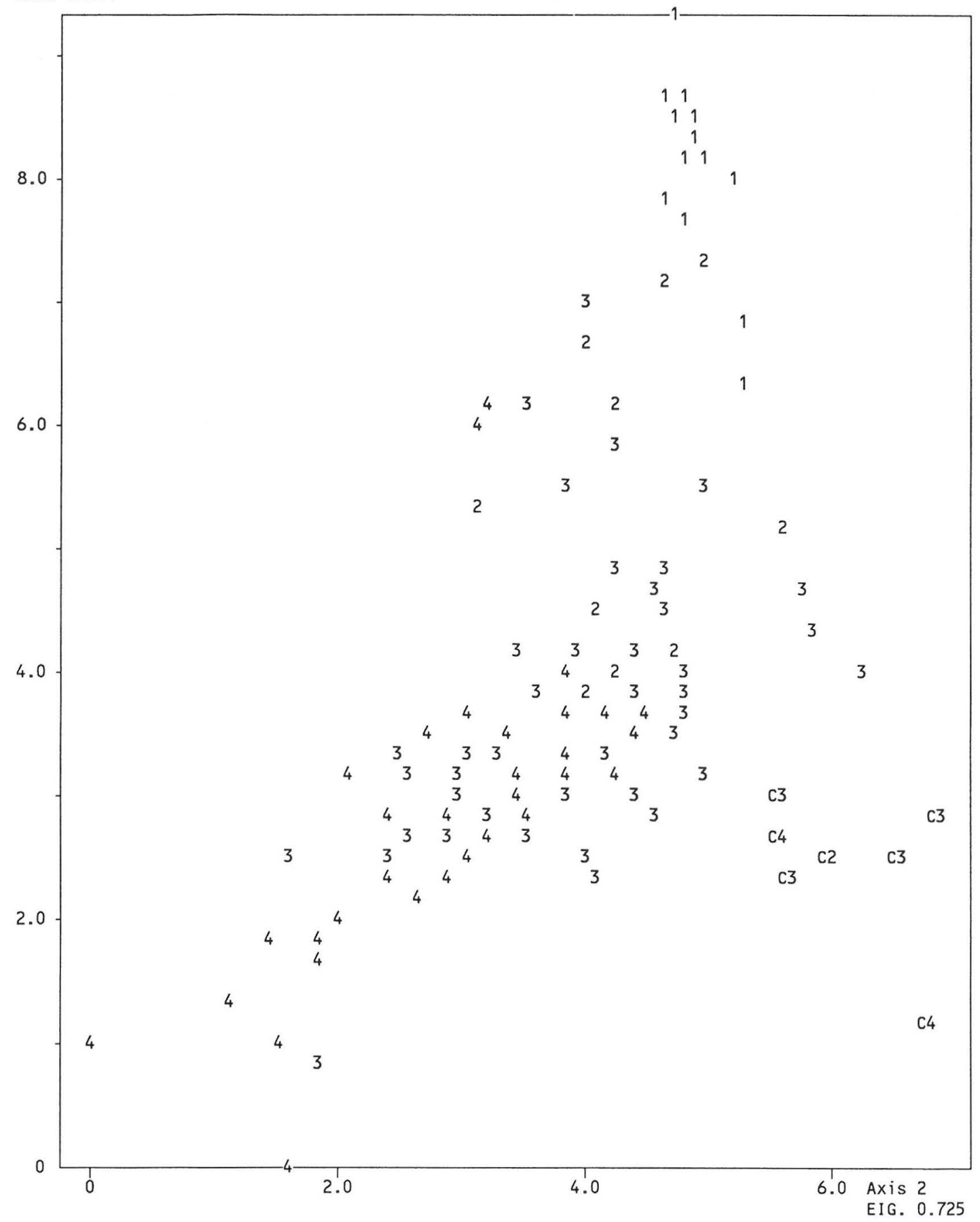

Fig. 19. DCA-ordination (the first two axes) of fallen, decomposing pine (Pinus sylvestris) trunks on the basis of the composition of wood-inhabiting Basidiomycetes (presence/absence). Numbers refer to sample trunks at different stages (1-5) of decay. Strongly charred trunks are marked with C. Species occurring on fewer than three sample trunks have been excluded from the ordination. 
Axis 1

EIG. 0.964

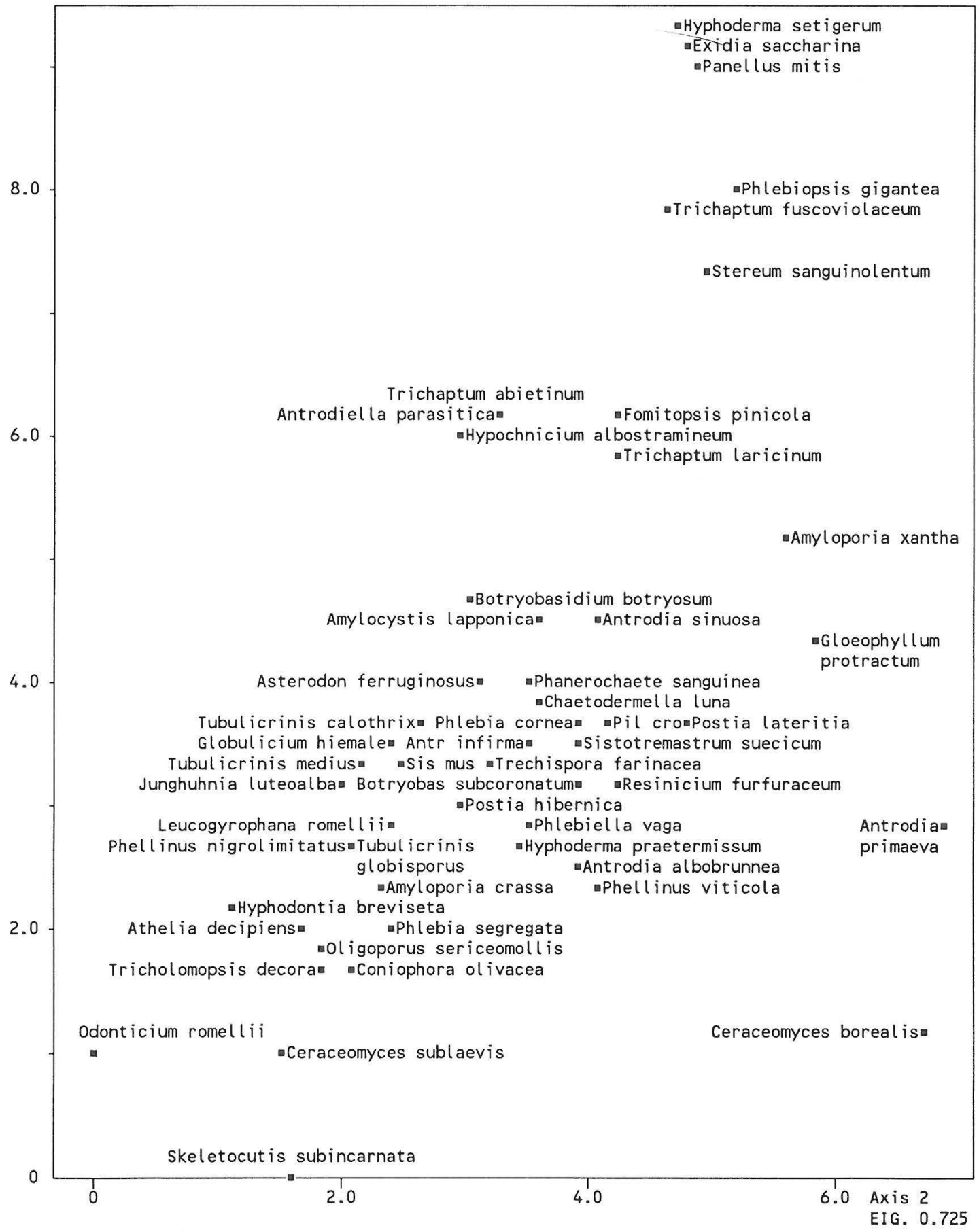

Fig. 20. DCA-ordination of Basidiomycetes (presence/absence) on fallen, decomposing trunks of Pinus sylvestris. Species occurring on fewer than three sample trunks have been excluded from the ordination. 


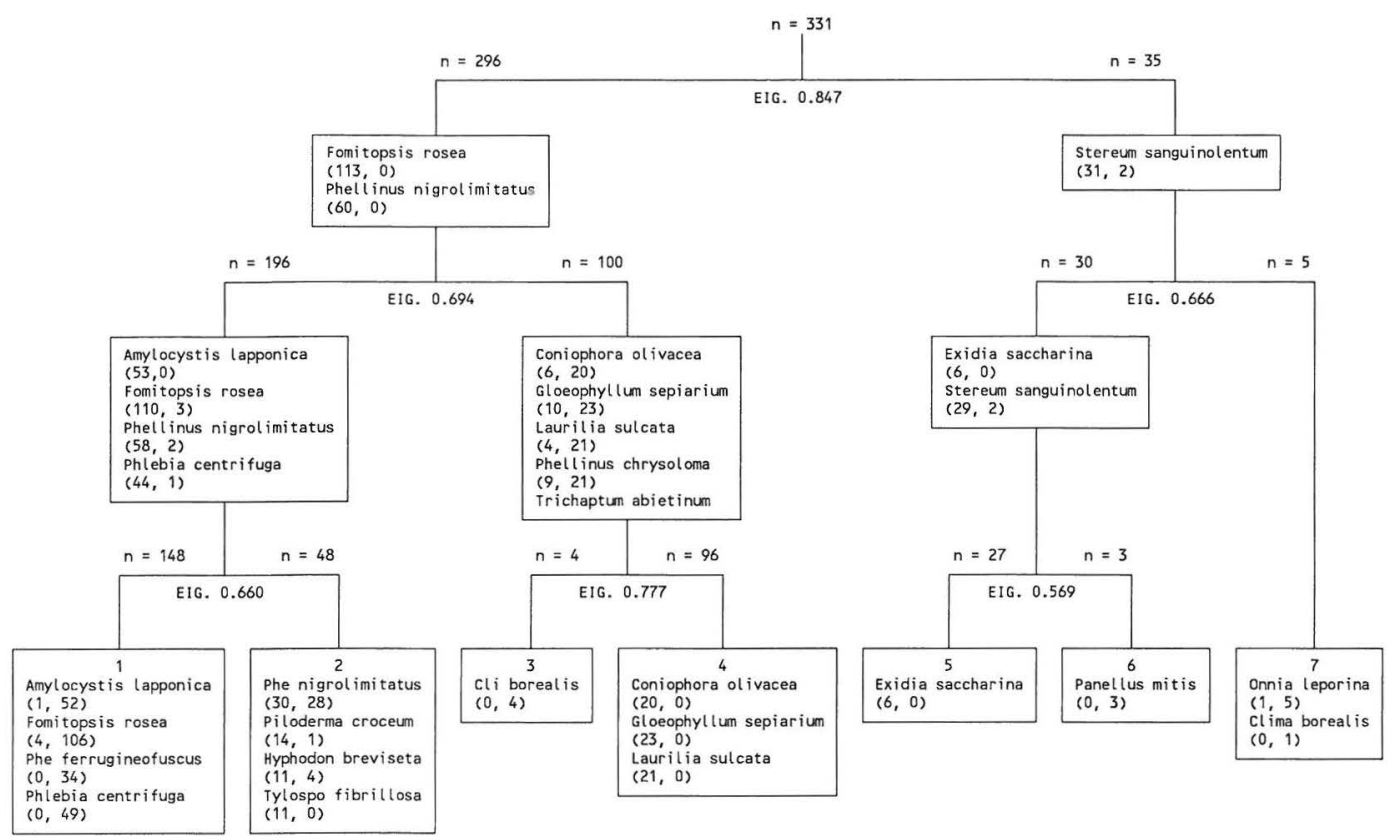

Fig. 21. Grouping of the decomposing spruce (Picea abies subsp. obovata) trunks in two-way indicator species analysis (TWINSPAN) on the basis of Basidiomycete species composition (presence/absence). The indicator species of each division are given. The numbers in parentheses show the frequencies of the species in the following subdivision of the trunks. Species occurring on fewer than three sample trunks have been excluded; $\mathrm{n}=$ number of trunks divided.

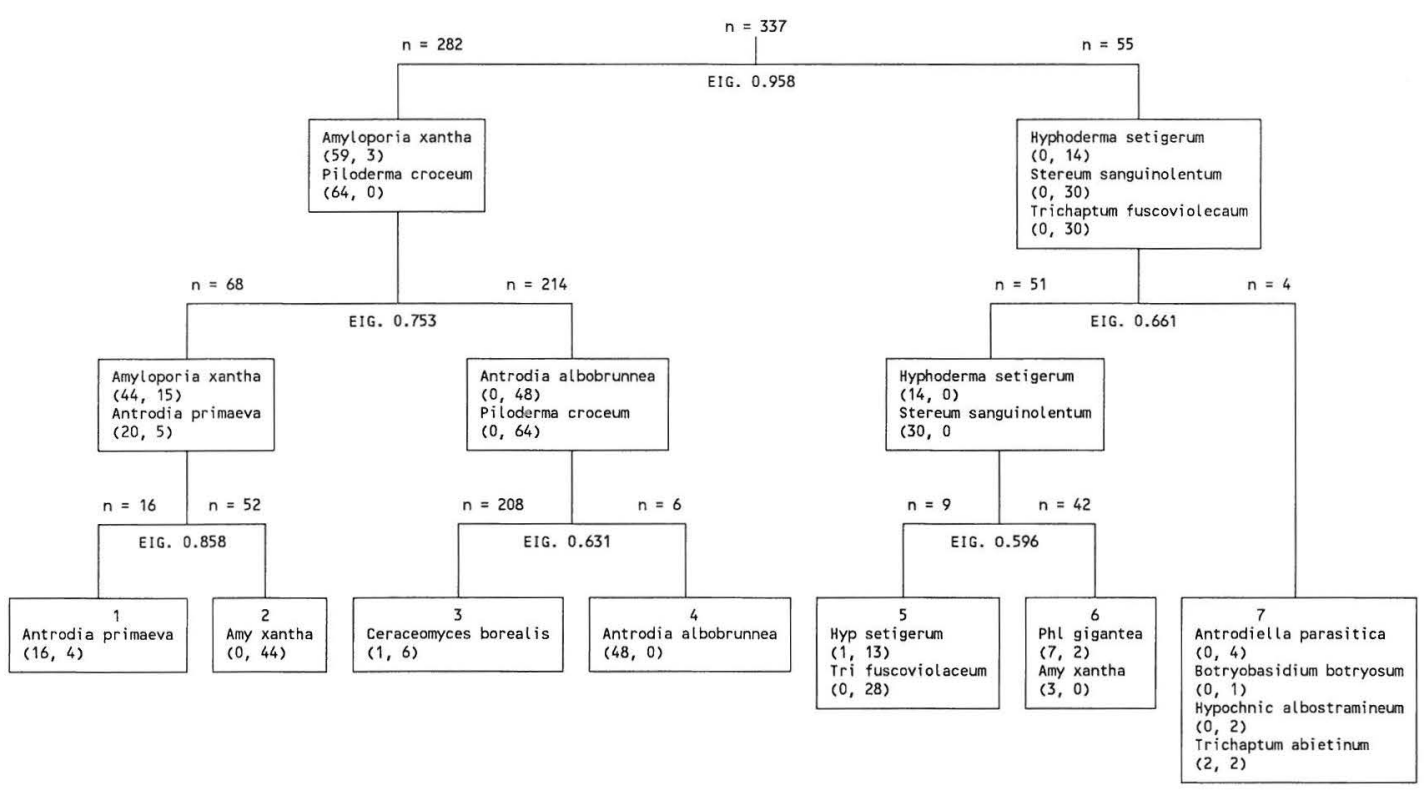

Fig. 22. Grouping of the decomposing pine (Pinus sylvestris) trunks in two-way indicator species analysis (TWINSPAN) on the basis of Basidiomycete species composition (presence/absence). The indicator species of each division are given. The numbers in parentheses show the frequencies of the species in the following subdivision of the trunks. Species occurring on fewer than three sample trunks have been excluded; $\mathrm{n}=$ number of trunks divided. 
primary decayers of spruce were white rot fungi. These play a vital role in the community dynamics of lignicolous fungi by opening up different successional pathways of decomposition. The ecologically highly variable niches occupied by white rot fungi emphasize the wide diversity (Otjen \& Blanchette 1986a, b, Eriksson et al. 1990) and heterogeneous phylogeny of these species (Ryvarden 1991).

As pointed out by Gilbertson (1981) and Ryvarden (1991), brown rot fungi are essential agents in the decomposition of coniferous trees in the boreal forest ecosystem. In my study area brown-rotters were clearly the dominant decomposers in dry habitats, in particular on pine. Many decorticated pine trunks were inhabited almost exclusively by brown-rotters. By contrast, white-rot fungi predominated in many spruce trunks, particularly in permanently wet localities. One explanation for this may be found in the physical and chemical differences e.g., the amount of wood sugar residues, of the two tree species (cf. Eriksson et al. 1990). As shown in this study, the number of brown rot fungi on decomposing trunks depends on the successional pathway of the decomposition (Table 25). Although the number of brown rot species was small compared to white rot fungi, most of them were frequently recorded, and the commonest species both on spruce and on pine were brown-rotters. Most brown rot fungi preferred intermediate stages of decay, which indicates their essential role at the main stages of decomposition.

The results of this study show that the species of wood-inhabiting fungi organize themselves more or less clearly according to the trunk diameter. They prefer either thick or thin trunks, and they are adapted to decompose only certain parts of the trunks. Many of the species that preferred thin trunks are frequently found in managed forests as well, while almost all the species that are adapted to decompose thick trunks are rare outside pristine or near-pristine forests, evidently because of the lack of suitable substrates. Likewise, the crown-inhabiting species (Tables 18 and 23), e.g., Panellus mitis, Phanerochaete sanguinea, Columnocystis abietina, Trichaptum abietinum, Antrodia serialis, Phellinus viticola and Gloeophyllum sepiarium, maintain viable populations in managed forests, whereas many of the species that are restricted to basal parts of the trunks cannot survive in there at all. All this indicates that large trunks are one of the most important substrates in maintaining species richness in the boreal forest ecosystem.

A characteristic feature of the mycoflora on the trunks was the small number of frequently occurring species. Although many of the rare species surely are rare, and the number of sample trunks may have been too small to fully reveal the ecology of all the species, the pattern that I discovered may partly have been due to incomplete field methods. Many corticioid fungi are ephemeral species which occupy only very small volumes of wood and produce basidiocarps that disappear quickly after sporulation, and many species (e.g., Botryobasidium Donk, Tubulicrinis Donk spp.) appear as very thin, small and more or less wood-coloured mycelial surfaces, which makes them inconspicuous even when fertile.

Not only small corticioid species but many easily observable polypores showed the same pattern. Eventhough the main decayer remained the same, each trunk, depending on some minor differences in terrain topography, tended to exhibit some properties of its own. Even very similar trunks lying next to each other seldom harboured exactly the same combination of species. Probably the great number of infrequent species indicates that a fallen tree trunk is a highly diverse ecological resource, a complex of units in which decomposition proceeds on many levels. On the other hand, the rare species were mostly recorded at late stages of decay, which indicates that several successional pathways are represented in the decomposition of fallen trunks.

Another feature characterizing fallen conifer trunks in the study area was the small number of agarics. According to my study, polypores are the most important wood-rotting element in northern boreal pristine forests. Many common pathogens of conifers in managed forests of the boreal zone were absent or very rare in the area. Heterobasidion annosum (Fr.) Bref. and Onnia tomentosa (Fr.) P. Karsten have a more southern distribution and were not found at all. Although over two thousands living pine trees were checked, Phellinus pini (Brot.: Fr.) A. Ames was recorded only nine times and Phaeolus schweinitzii (Fr.) Pat. only once. The genus Armillaria Fr. seems to be restricted to fallen birch (Betula) trunks in the study area. 


\section{Diversity}

If the almost completely decayed trunks (D.S. 5) are excluded, the number of species occurring on the trunks of both spruce and pine increased noticeably with the stage of decomposition. A fairly similar successional trend, although with a different species composition, was noticed on decomposing spruce trunks in Norway (Framstad et al. 1992, Bendiksen \& Høiland 1994). The great differences in the amounts of species on freshly fallen and extensively decayed trunks reflect the considerable physical and chemical changes taking place in decomposing wood (cf. Eriksson et al. 1990). A freshly fallen, undecayed trunk is a fairly homogeneous substrate. During the decomposition the activities of decomposers locally change its structure, moisture and chemistry, thus creating new niches for fungi. In addition, because several successional pathways exist in the decomposition of fallen trunks, and the species compositions of the fungi vary according to the pathway, a variety of microhabitats characteristic of each pathway will be created in extensively decayed trunks.

Each decay stage harboured a number of species that were not found at all on trunks at other stages of decay. Fresh windfalls (D.S. 1) of spruce harboured seven such species, and extensively decayed trunks (D.S. 4) 25. Freshly uprooted pine trunks (D.S. 1) harboured three species which were not recorded on trunks at other stages of decay, and the trunks at both decay stages 3 and 4 harboured 19 such species. Accordingly, each decay stage harbours a characteristic species composition. However, the trunks at late stages of decay (D.S. 4, in particular) maintain exceptionally diverse species combinations and serve as important mycelium and spore banks for many saprotrophic fungi. Most of these late-succession species are known to prefer old forest habitats and have diminished in number because of forestry. Many of them have been classified as threatened in Nordic countries (Bendiksen \& Høiland 1992, Rassi et al. 1992, Kotiranta \& Niemelä 1993, Hallingbäck 1994).

\section{Community structure and development}

The fungi were found to have specific preferences for certain stages of wood decomposition. Similar results have been obtained by Lange (1992) on the sequence of fungi on beech logs, and by Bendiksen and Høiland (1994) who studied the effects of forestry on wood-rotting fungi on spruce trunks in Central Norway. However, the study on filamentous fungi and yeasts on decomposing logs of Pseudotsuga menziesii in Oregon, U.S. carried out by Crawford et al. (1990), by using isolates from cross-sections of logs, did not reveal a strong successional trend of species. One explanation for this may be that only trunks at intermediate stages of decay were included in their study. As demonstrated by Boddy (1992) the species of wood-inhabiting fungi replace one other in a characteristic sequence during the decomposition of wood, altering the physical structure, moisture, acidity and nutrient contents of the wood. In its altered condition the wood becomes suitable for the establishment and colonization of successor fungi (secondary resource capture, see Rayner \& Boddy 1988a). The distinct successional orders of species that develop on decaying trunks show that lignicolous Basidiomycetes differ greatly from each other in their substratum requirements and in their competitive abilities at different stages of decomposition. This variety makes great demands on the research on fungal systematics, because taxonomically collective species concepts (e.g., the genera Antrodiella Ryvarden \& Johan. and Skeletocutis Kotl. \& Pouzar) tend to mask and obscure differences of this kind.

The results of this study have shown that the fungal composition in a conifer trunk is most closely tied to the stage of decomposition. In addition, many other trunk characteristics contribute to the structure and dynamics of fungal communities. The history of fungal infections preceding the tree fall has a strong impact, as do the base diameter and type of stem breakage. Because bark reduces evaporation from fallen trunks and thus is a moderating factor in controlling the moisture content of wood, its amount affects the microclimatical conditions inside wood. Physical and chemical properties of the host tree species and the microclimate of the growth site govern the basic trends in the community development of wood-inhabiting fungi. However, the first stages in the tree trunk decomposition greatly depend on the way the tree died. Primary decayers significantly alter the structure and chemistry of wood, and in this way 
affect the compositions of fungal communities in later stages of succession.

Different pioneers open different successional pathways. Subsequent species are affected and selected according to their predecessors, and in their turn have a strong influence on what species will follow (replacement interactions, see Rayner \& Boddy 1988a). For example, by significantly changing the quality of wood the base-inhabiting primary decayers on spruce, i.e., Climacocystis borealis, Fomitopsis pinicola, F. rosea, Onnia lepporina and, in particular, Phellinus chrysoloma and Trichaptum laricinum, greatly affect the ability of other fungi to colonize the trunk. Furthermore, evidently because they require particular chemical and physical properties in the wood, some rare and threatened saprotrophic polypores only inhabit trunks already decayed by specific species (Niemelä et al. 1995). These successors emerge only after the preceding fungus has died. Examples on spruce are Pycnoporellus fulgens and Antrodiella citrinella, which follow Fomitopsis pinicola, Skeletocutis carneogrisea and Antrodiella parasitica, which in turn depend on Trichaptum spp., and Piloporia sajanensis which grows only on trunks that have been extensively decayed by Trichaptum laricinum.

All this suggests that strong physiological and ecological ties, or even obligatory dependencies exist between the late saprotrophs and the early colonizers. Each species has a unique ecological role in the process of decomposition (e.g., Rayner \& Hedges 1982, Boddy \& Rayner 1983a). Some species are active decayers that occupy large volumes of the trunks, whereas others are highly local, or utilize the last remnants of decaying wood, and may even depend on metabolic products of other fungi or exist in parasitic relationships with them (Cooke \& Rayner 1984, Rayner et al. 1987, Rayner \& Boddy 1988a, Renvall \& Niemelä 1992b, Jeffries \& Young 1994). As demonstrated by Swift (1987) and also shown in this study, communities of wood-rotting fungi are dynamic entities that change continuously in space and time. While often exhibiting fairly constant species compositions, they never reach a state of equilibrium.

Although the succession of wood-inhabiting fungi, i.e. the replacement of mycelia of one species by mycelia of another, has been demonstrated in many studies (summarized by Rayner \& Boddy 1988a and Boddy 1992), very little is known about how the succession of mycelia is reflected in the basidiocarp production. Presumably the internal mycelial dynamics and the succession of basidiocarps differ widely with the fungus species, and comparative studies are needed to evaluate these differences. For purposes of the present study, suffice it to note that basidiocarps are the best indicators of the reproductive ability of the fungi, and data based on the fruit body production give the most reliable information on the spatial dynamics of the fungi outside the wood.

\section{Decomposing trunks as resource units for} lignicolous fungi

During decomposition, fallen tree trunks undergo structural and chemical changes in a generally recognized order. The trunk becomes decorticated and collapses against the ground. The wood becomes softened and cracked, and the density steadily decreases (Christensen 1984, Sollins et al. 1987). All this together with the activities of decomposing organisms increases the moisture content. Over the years, trunks become more or less covered with bryophytes and lichens (e.g., Söderström 1988), which then increase the water-holding capacity of the wood and reduce evaporation. As indicated by the results of the present study and shown by Dix (1985) and Sollins et al. (1987), the moisture content of wood in fallen trunks increases markedly during decomposition. Also the nutrient contents of wood differ significantly with the stage of decay (Grier 1978, Lambert et al. 1980, Sollins et al. 1987). The amount of gaseous carbon dioxide increases inside decaying wood, until fragmentation of the wood enhances ventilation (Hintikka \& Korhonen 1970). Both the chemical and physical conditions of wood are thus closely related to the stage of decomposition of the trunk. Forest fires create new substrates for fungi to invade. Through changing the quality of wood, burning totally changes the pathway of decomposition.

Thick and thin trunks of the same tree species differ ecologically from each other in many ways. Because of the slow growth rate, wood density is usually higher in thin understorey trees than in rapidly-growing upper canopy trees. After falling a thin (and light) trunk will typically remain for many years, lying on its root plate and 
branches, before collapsing against the ground. During that time it may undergo periods of harsh drought. However, after it has completely fallen its moisture content increases and the whole trunk is rapidly invaded by decayers, many of them evidently via mycelial cords (or rhizomorphs) from the soil (Thompson \& Boddy 1983, Coates \& Rayner 1985b, Dowson et al. 1986, 1988, Chapela et al. 1988, Boddy 1993). Eventually the trunk will be overgrown by bryophytes and lichens (ground floor species). After ground contact, thin trunks are relatively rapidly decomposed.

When trees with heavy and thick trunks fall because of decay, they usually immediately assume ground contact throughout their length. Their large volume slows down decomposition and the slow decomposition rate is evidently an important factor in maintaining high species diversity of fungi. Many threatened species, e.g., Amyloporia crassa, Antrodia infirma and Skeletocutis stellae, seem to depend on the slow decay process of large-volume trunks. If a tree with a thick trunk is uprooted because of strong wind, it may stay a few years uplifted on its root plate. However, at least the top third of the trunk will be in direct contact with the ground. Voluminous trunks have thick bark and retain it for a fairly long time, whereas thin trunks become decorticated much sooner after falling. Greater wood volume maintains more stable microclimatical conditions (temperature, moisture) inside the trunk, while thin trunks undergo rapid and dramatic changes of many kinds (Boddy 1983). All in all, a large trunk offers much greater ecological potential for woodrotting fungi than what a thin trunk does. It offers a whole series of niches in both horizontal and vertical direction, and in relation to the distance from the surface. As indicated by the results of this study, large trunks maintain higher species diversity than thin trunks.

To conclude: Varying with tree species, the microclimate of the site, stage of decomposition, size, history of fungal infections, type of stem breakage and amount of bark, decomposing tree trunks offer a rich variety of ecological niches for lignicolous organisms to invade. A fallen trunk should be understood as a temporally changing, highly heterogeneous and spatially discontinuous resource unit. It is a substrate in a dynamic state, which finally will be eradicated by its own inhabitants. Although it is an independent unit and undergoing internal successional processes of its own, the disturbance caused to the surrounding forest vegetation appreciably affects its properties, making it a vulnerable habitat.

Besides serving as a host for a great number of lignicolous fungi, invertebrates, bryophytes and lichens, decomposing wood plays an essential role in forest regeneration biology. According to Larsen et al. (1980) brown rot residues make up a considerable part of the humus layer in boreal forests and increase the water-holding capacity of soil. Furthermore, decayed wood is an important substrate for ectomycorrhizal activity. As has been shown in many studies in North America (Harvey et al. 1976, 1979, Kropp 1982a, b, Kropp \& Maser 1982), rotten wood supports a substantial portion of the total number of ectomycorrhizae in the forest floor. As seedbeds of trees decomposing trunks actively contribute to forest regeneration (Harmon et al. 1986, Harmon \& Franklin 1989). According to Larsen et al. (1980) trunks that have been extensively decayed by brown rotters, in particular, are important sites for tree seedlings. In the study area, old spruce trunks fairly often were serving as nurse logs.

Some corticiaceous species (Amphinema byssoides, Byssocorticium terrestris, Piloderma croceum, $P$. byssinum, $P$. olivaceum) that were found on sample trunks are important mycorrhiza formers (Froidevaux et al. 1978, Ginns \& Lefebvre 1993, Erland et al. 1994). Their frequency of occurrence was highest on extensively decayed trunks (decay stages 4-5). Piloderma croceum was particularly common, being found on $24.3 \%$ of the pine trunks at the decay stages 4 and 5 . Although Tanesaka et al. (1993) have shown that some mycorrhizal fungi are not able to degrade wood, the frequent occurrence of corticiaceous ectomycorrhizal fungi on decomposing trunks in this study indicates that they contribute in decomposing the last remnants of fallen trunks. Thus, by linking the decomposition of wood with the germination of tree seeds and the early growth of seedlings, they evidently play an essential role in the natural regeneration dynamics of boreal forests. 


\section{Rate of decomposition}

In boreal climate the complete decomposition of a large, fallen, pine or spruce will take over 100 years. Estimates range from 70 years in southern boreal Sweden (Hytteborn \& Packham 1987) to 200 years or even more in northern boreal forests (Hofgaard 1993). In addition to the major climatic factors, also microclimatical conditions of the site, frequency of forest fires, physical and chemical properties of the tree species, age and size of the tree and type of stem breakage have their influence on the decomposition processes. Many biotic factors play an essential role in the decomposition, too. The activities of bark beetles and other insects, and woodpeckers, particularly the Three-toed Woodpecker (Picoides tridactylus L.) affect how long a conifer trunk retains its bark.

The major factor affecting the rate of decomposition of a fallen trunk is the primary decayer. Phellinus chrysoloma on spruce and Trichaptum laricinum on both spruce and pine are rapid invaders that aggressively occupy large volumes of the trunks. The wood decayed by these fungi rapidly becomes softened, and complete decomposition occurs much more quickly than in trunks primarily decayed by slow-working fungi such as Fomitopsis pinicola. Accordingly, estimates of the decomposition rates should always include comprehensive and detailed definitions of the ecological factors mentioned above, and data on decomposers.

\section{Conservation of wood-inhabiting fungi}

As shown by Bader et al. (1995) and indicated by qualitative ecological data in many mycofloristic papers (Kotiranta \& Niemelä, Renvall et al, 1991b, Niemelä et al. 1992, Niemelä 1994b), the species richness is much lower in managed forests than in pristine or near-pristine forests. It has been estimated in Finland that, depending on the degree of logging, up to $80 \%$ of the species of wood-inhabiting fungi disappear because of forestry. The main reason for this is the lack of suitable substrates in managed forests. Modern forestry eradicates fallen tree trunks, and the remaining decomposing wood chiefly consists of thin, freshly cut trunks, stumps and small twigs. As shown in this study such woody material supports only a tiny portion of the total myco- flora involved in the natural dynamics of wood decomposition. Many fungi, in particular species that prefer large trunks, and many late-successional saprotrophs are now threatened as a result. Because the remaining pristine or nearpristine forests have become more and more fragmented and are now almost always isolated by vast areas of managed forests, the survival of many rare fungi ultimately depends on their dispersal abilities and colonization strategies (metapopulation dynamics, see Hanski 1991, Hanski \& Gilpin 1991).

The lack of quantitative studies on the community and population ecology of wooddecomposing fungi makes them especially problematic in the conservation biology of boreal primeval forests (cf. Esseen et al. 1992, Haila 1994). In many studies focused on lignicolous organisms it has been concluded that the dynamics of wood decomposition plays a crucial role in maintaining a high species diversity in boreal forests. Wood-inhabiting fungi are key organisms when it comes to analysing and interpreting the dynamics and conservational value of old forests. On the basis of the present study it is evident that the conservation of lignicolous fungi will depend upon the depth with which we understand fungal community development and the decomposition dynamics of fallen tree trunks.

Acknowledgements. I wish to thank my supervisor Prof. Tuomo Niemelä for his expert guidance and friendly support at all stages of the work. The manuscript was much improved by the constructive comments of Prof. Niemelä and Dr. Jan Stenlid (Uppsala). Dr. Pekka Pakarinen kindly assisted with the numerical treatment of the data, and Mr. Urmas Kõljalg (Tartu) provided valuable help in the identification of the tomentelloid fungi. The research was financed by the Academy of Finland (project No. 1808, 1011799).

\section{References}

Aanstad, S. \& Ryvarden, L. 1987: Aphyllophorales on wooden fences in Norway. - Windahlia 17:49-54.

Andersson, L.I. \& Hytteborn, H. 1991: Bryophytes and decaying wood -- a comparison between managed and natural forest. - Holarctic Ecol. 14:21-130.

Ahti, T., Hämet-Ahti, L. \& Jalas, J. 1968: Vegetation zones and their sections in northwestern Europe. Ann. Bot. Fennici 5:169-211.

Angelstam, P. \& Mikusiński, G. 1994: Woodpecker assemblages in natural and managed boreal and hemiboreal forests -- a review. -- Ann. Zool. Fennici 31:157-172.

Bader, P., Jansson, S. \& Jonsson, B.G. 1995: Wood- 
inhabiting fungi and substrate decline in selectively logged boreal spruce forests. - Biol. Conserv. (submitted)

Bendiksen, E. \& Høiland, K. 1992: Red list of threatened macromycetes in Norway. - Directorate for Nature Management Report 1992-6:31-42.

Bendiksen, E. \& Høiland, K. 1994: Modern forestry and effect on biodiversity of wood-rotting fungi. Fifth International Mycological Congress, August 1421, 1994, Vancouver, BC, Abstracts: 14.

Boddy, L. 1983: Microclimate and moisture dynamics of wood decomposing in terrestrial ecosystems. - Soil Biol. Biochem. 15:149-157.

Boddy, L. 1992: Development and function of fungal communities in decomposing wood. In: Wicklow, D.T. \& Carroll, C.G. (eds.), The fungal community. Its organization and role in the ecosystem: pp. 749782. - Marcel Dekker, New York.

Boddy, L. 1993: Saprotrophic cord-forming fungi: warfare strategies and other ecological aspects. -- Mycol. Res. 97:641-655.

Boddy, L., Bardsley, D.W. \& Gibbon, O.M. 1987: Fungal communities in attached ash branches. New Phytol. 107:143-154.

Boddy, L. \& Rayner, A.D.M. 1983a: Ecological roles of basidiomycetes forming decay communities in attached oak branches. - New Phytol. 93:77-88.

Boddy, L. \& Rayner, A.D.M. 1983b: Origins of decay in living deciduous trees: the role of moisture content and a re-appraisal of the expanded concept of tree decay. - New Phytol. 94:623-641

Boddy, L. \& Rayner, A.D.M. 1983c: Mycelial interactions, morphogenesis and ecology of Phlebia radiata and Phlebia rufa from oak. - Trans. British Mycol. Soc. 80:437-448.

Butcher, J.A. 1968: The ecology of fungi infecting untreated sapwood of Pinus radiata. - Canadian J. Bot. 46:1577-1589.

Chamuris, G.P. \& Falk, S.P. 1987: The population structure of Peniophora rufa in an aspen plantation. - Mycologia 79:451-457.

Chapela, I.H. \& Boddy, L. 1988a: Fungal colonization of attached beech branches 1. Early stages of development of fungal communities. - New Phytol. 110:39-45.

Chapela, I.H. \& Boddy, L. 1988b: Fungal colonization of attached beech branches 2. Spatial and temporal organization of communities arising from latent invaders in bark and functional sapwood, under different moisture regimes. — New Phytol. 110:4757.

Chapela, I.H. \& Boddy, L. 1988c: The fate of early fungal colonizers in beech branches decomposing on the forest floor. - FEMS Microbiol. Ecol. 53:273-284.

Chapela, I.H., Boddy, L. \& Rayner, A.D.M. 1988: Structure and development of fungal communities in beech logs four and a half years after felling. FEMS Microbiol. Ecol. 53:59-70.

Chase, T.E. \& Ullrich, R.C. 1983: Sexuality, distribution, and dispersal of Heterobasidion annosum in pine plantations of Vermont. - Mycologia 75:825-831.

Chesson, P.L. \& Case, T.J. 1986: Overview: Nonequilibrium community theories: chance variability, history and coexistence. In: Diamond, J.M. \& Case, T.J. (eds.), Community ecology: pp. 229-239. - Harper \& Row, New York.

Christensen, O. 1984: The state of decay of woody litter determined by relative density. - Oikos 42:211219.

Coates, D. \& Rayner A.D.M. 1985a: Fungal population and community development in beech logs. 1 . Establishment via the aerial cut surface. - New Phytol. 101:153-171.

Coates, D. \& Rayner A.D.M. 1985b: Fungal population and community development in beech logs. 2 . Establishment via the buried cut surface. - New Phytol. 101:173-181.

Coates, D. \& Rayner A.D.M. 1985c: Fungal population and community development in beech logs. 3. Spatial dynamics, interactions and strategies. New Phytol. 101:183-198.

Cooke, R.C. \& Rayner, A.D.M. 1984: Ecology of saprotrophic fungi. - 415 pp. Longman, London and New York.

Crawford, R.H., Carpenter, S.E. \& Harmon, M.E. 1990: Communities of filamentous fungi and yeast in decomposing logs of Pseudotsuga menziesii. Mycologia 82:759-765.

DeAngelis, D.L. \& Waterhouse, J.C. 1987: Equilibrium and nonequilibrium concepts in ecological models. - Ecol. Monogr. 57:1-21.

Dix, N.J. 1985: Changes in relationship between water content and water potential after decay and its significance for fungal successions. - Trans. British Mycol. Soc. 85:649-653.

Dowson, C.G., Rayner, A.D.M. \& Boddy, L. 1986: Outgrowth patterns of mycelial cord-forming basidiomycetes from and between woody resource units in soil. - J. Gen. Microbiol. 121:203-211.

Dowson, C.G., Rayner, A.D.M. \& Boddy, L. 1988: The form and outcome of mycelial interactions involving cord-forming decomposer basidiomycetes in homogeneous and heterogeneous environments. New Phytol. 109:423-432.

Eriksson, J. 1958: Studies in the Heterobasidiomycetes and Homobasidiomycetes-Aphyllophorales of Muddus national park in North Sweden. - Symb. Bot. Upsal. 16:1-172.

Eriksson, J. \& Ryvarden, L. 1973: The Corticiaceae of North Europe 2. Aleurodiscus-Confertobasidium. - Pp. 55-261. Fungiflora, Oslo.

Eriksson, J. \& Ryvarden, L. 1975: The Corticiaceae of North Europe 3. Coronicium-Hyphoderma. - Pp. 887-546. Fungiflora, Oslo.

Eriksson, J. \& Ryvarden, L. 1976: The Corticiaceae of North Europe 4. Hyphodermella-Mycoacia. - Pp. 547-886. Fungiflora, Oslo.

Eriksson, J., Hjortstam, K. \& Ryvarden, L. 1978: The Corticiaceae of North Europe 5. MycoaciellaPhanerochaete. - Pp. 887-1047. Fungiflora, Oslo.

Eriksson, J., Hjortstam, K. \& Ryvarden, L. 1981: The Corticiaceae of North Europe 6. PhlebiaSarcodontia. - Pp. 1051-1276. Fungiflora, Oslo.

Eriksson, J., Hjortstam, K. \& Ryvarden, L. 1984: The Corticiaceae of North Europe 7. SchizoporaSuillosporium. - Pp. 1277-1449. Fungiflora, Oslo. 
Eriksson, J. \& Strid, A. 1969: Studies in the Aphyllophorales (Basidiomycetes) of northern Finland. - Ann. Univ. Turku. AII 40 (Rep. Kevo Subarctic Sta. 4):112-158.

Eriksson, K.L., Blanchette, R.A. \& Ander, P. 1990: Microbial and enzymatic degradation of wood and wood components. - 407 pp. Springer-Verlag, Berlin, etc.

Erkkilä, R. \& Niemelä, T. 1986: Polypores in the parks and forests in the City of Helsinki. - Karstenia 26: $1-40$.

Erland, S., Henrion, B., Martin, F., Glover, L.A. \& Alexander, I.J. 1994: Identification of the ectomycorrhizal basidiomycete Tylospora fibrillosa Donk by RFLP analysis of the PCRamplified ITS and IGS regions of ribosomal DNA. - New Phytol. 126:525-532.

Esseen, P.A., Ehnström, B., Ericson, L. \& Sjöberg, K. 1992: Boreal forests - the focal habitats of Fennoscandia. In: Hansson, L. (ed.), Ecological principles of nature conservation, applications in temperate and boreal environments: pp. 252-325. - Elsevier, London \& New York.

Framstad, E., Bendiksen, E., Flatberg, K.I., Frisvoll, A., Holien, H., Høiland, K., Prestø, T. \& Svalastog, D. 1992: Effekten av fragmentering og kvalitetsendring i barskog på kryptogamer. Skogforsk 13/92:4-15.

Frankland, J.C. 1992: Mechanisms in fungal successions. In: Wicklow, D.T. \& Carroll, C.G. (eds.), The fungal community. Its organization and role in the ecosystem: pp. 383-401. - Marcel Dekker, New York.

Franklin, J.F., Shugart, H.H. \& Harmon, M.E. 1987: Tree death as an ecological process. - BioScience 37:550-556.

Froidevaux, L., Amiet, R. \& Jaquenoud-Steinlin, M. 1978: Les Hyménomycétes résupinés mycorrhiziques dans le bois pourri. - Schweiz. Z. Pilzkunde 56:9-14.

Gilbertson, R.L. 1981: North American wood-rotting fungi that cause brown rots. - Mycotaxon 12:372416.

Giller, P.S. \& Gee, J.H.R. 1987: The analysis of community organization: The influence of equilibrium, scale and terminology. In: Gee, J.H R. \& Giller, P.S. (eds.), Organization of communities past and present: pp. 519-542. - Blackwell, Oxford, etc.

Ginns, J. 1978: Leucogyrophana (Aphyllophorales): identification of species. - Canadian J. Bot. 56:1953-1973.

Ginns, J. \& Freeman, G.W. 1994: The Gloeocystidiellaceae (Basidiomycota, Hericiales) of North America.- Bibl. Mycol. 157:1-118.

Ginns, J. \& Lefebvre, M.N.L. 1993: Lignicolous corticioid fungi (Basidiomycota) of North America. Systematics, distribution, and ecology. Mycologia Memoir 19:1-247.

Graham, S.A. 1925: The felled tree trunk as an ecological unit. - Ecology 6:397-416.

Grier, C.C. 1978: A Tsuga heterophylla-Picea sitchensis ecosystem of coastal Oregon: decomposition and nutrient balances of fallen logs. - Canadian J. Forest Res. 8:198-206.

Griffin, D.M. 1977: Water potential and wood decay fungi. - Ann. Rev. Phytopathol. 15:319-329.

Griffith, G.S. \& Boddy L. 1990: Fungal decomposition of attached angiosperm twigs 1 . Decay community development in ash, beech and oak twigs. - New Phytol. 116:407-415.

Haila, Y. 1994: Preserving ecological diversity in boreal forests: ecological background, research, and management. - Ann. Zool. Fennici 31:203-217.

Haila, Y., Niemelä, P. \& Kouki, J. (eds.) 1994: Metsätalouden ekologiset vaikutukset boreaalisissa havumetsissä. Effects of management on the ecological diversity of boreal forests. - Finnish Forest Res. Inst., Res. Papers 482:1-123.

Hallaksela, A.-M. 1993: Early interactions of Heterobasidion annosum and Stereum sanguinolentum with non-decay fungi and bacteria following inoculation into stems of Picea abies. European J. Forest Pathol. 23:416-430.

Hallaksela, A.-M. 1994: Early microbial community develpoment in stems of Picea abies inoculated with characterised decay fungi, non-decay fungi and bacteria. - Finnish Forest Res. Inst., Res. Papers 485: 1-105.

Hallaksela, A.-M. \& Salkinoja-Salonen, M. 1992: Bacteria inhabiting artificially inoculated xylem of Picea abies. - Scand. J. Forest Res. 7:165-175.

Hallenberg, N. 1985a: The Lachnocladiaceae and Coniophoraceae of North Europe. - 96 pp. Fungiflora, Oslo.

Hallenberg, N. 1985b: On the Hypochnicium eichleri complex (Basidiomycetes). - Mycotaxon 24:431436.

Hallingbäck, T. 1994: Ekologisk katalog över storsvampar. SNV Report 4313. - 213 pp. Swedish National Environmental Protection Agency, Uppsala.

Hämet-Ahti, L. 1983: Human impact on closed boreal forest (taiga). In: Holzner, W., Werger, M. J. A. \& Ikusima, I. (eds.), Man's impact on vegetation: pp. 201-211. - W. Junk Publishers, The Hague, etc.

Hämet-Ahti, L., Suominen, J., Ulvinen, T., Uotila, P. \& Vuokko, S. (eds.) 1986: Retkeilykasvio. -598 pp. Suomen Luonnonsuojelun Tuki, Helsinki.

Hämet-Ahti, L., Palmén, A., Alanko, P. \& Tigerstedt, P.M.A. 1992: Suomen puu- ja pensaskasvio. — 373 pp. Dendrologian Seura, Helsinki.

Hanski, I. 1991: Single species metapopulation dynamics: concepts, models and observations. - Biol. J. Linnean Soc. 42:17-38.

Hanski, I. \& Gilpin, M. 1991: Metapopulation dynamics: brief history and conceptual domain. - Biol. J. Linnean Soc. 42:3-16.

Hansen, L. \& Knudsen, H. 1992: Nordic Macromycetes 2. - 474 pp. Nordsvamp, Copenhagen.

Hansson, L. (ed.) 1992: Ecological principles of nature conservation, applications in temperate and boreal environments. - $436 \mathrm{pp}$. Elsevier, London \& New York.

Harmon, M.E. \& Franklin, J.F. 1989: Tree seedlings on logs in Picea-Tsuga forests of Oregon and 
Washington. - Ecology 70:48-59.

Harmon, M.E., Franklin, J.F., Swanson, F.J., Sollins, P., Gregory, S.V., Lattin, J.D., Anderson, N.H., Cline, S.P., Aumen, N.G., Seddell, J.R., Lienkaeper, G.W., Cromack, K. Jr. \& Cummins, K.W. 1986: Ecology of coarse woody debris in temperate ecosystems. - Adv. Ecol. Res. 15:133302.

Harvey, A.E., Larsen, M.J. \& Jurgensen, M.F. 1976: Distribution of ectomycorrhizae in mature Douglas fir -larch forest soil in western Montana. - Forest Sci. 22:393-398.

Harvey, A.E., Larsen, M.J. \& Jurgensen, M.F. 1979: Comparative distribution of ectomycorrhizae in soils of three western Montana forest habitat types. - Forest Sci. 25:350-358.

Hill, M.O. 1979: TWINSPAN — a FORTRAN program for arranging multivariate data in an ordered twoway table by classification of the individuals and attributes. - $48 \mathrm{pp}$. Cornell Univ., Ithaca, NY.

Hill, M.O. \& Gauch, H.G. 1980: Detrended correspondence analysis: an improved technique. - Vegetatio 42:47-58.

Hintikka, V., Korhonen, K. 1970: Effects of carbon dioxide on the growth of lignicolous and soilinhabiting Hymenomycetes. - Comm. Inst. Forest. Fenniae 69:1-28.

Hintikka, V. 1993: Occurrence of edible fungi and other macromycetes on tree stumps over a sixteen-year period. - Acta Bot. Fennica 149:11-17.

Hjortstam, K. 1981: Notes on Aphyllophorales (Basidiomycetes) from Omberg, Östergötland, Sweden. - Windahlia 11:39-55.

Hjortstam, K. \& Johannesen, E. 1980: Annotated list of the alpine wood fungus flora in Norway. Aphyllophorales and Myxomycetes in Dovrefjekk National Park. - Windahlia 10:15-45.

Hjortstam, K., Larsson, K.-H. \& Ryvarden, L. 1987. The Corticiaceae of North Europe 8. Phlebiella, Thanatephorus-Ypsilonidium. - Pp. 1450-1631. Fungiflora, Oslo.

Hjortstam, K., Larsson, K.-H. \& Ryvarden, L. 1988 : The Corticiaceae of North Europe 1. Introduction and keys. - Pp. 1-59. Fungiflora, Oslo.

Hofgaard, A. 1993: 50 years of change in a Swedish boreal old-growth Picea abies forest. - J. Veget. Sci. 4:773-782.

Hytteborn, H. \& Packham, J.R. 1987: Decay rate of Picea abies $\operatorname{logs}$ and the strom gap theory: a reexamination of Sernander plot III, Fiby Urskog, central Sweden. - Arboric. J. 11:299-311.

Jahn, H. 1962: Pilzewuchs an Fichtenstümpfen (Picea) in Westfälischen Gebirgen. - Westfälische Pilzbriefe 3:110-122.

Jahn, H. 1966: Pilzgesellschaften an Populus tremula. Z. Pilzkunde 32:26-42.

Jahn, H. 1968: Pilze an Weisstanne (Abies alba). Westfälische Pilzbriefe 7:17-40.

Jeffries, P. \& Young, T.W.K. 1994: Interfungal parasitic relationships. - $296 \mathrm{pp}$. CAB International, Oxon.

Johansen, I. \& Ryvarden, L. 1977: Wood-inhabiting fungi (Aphyllophorales, Homobasidiomycetes) in two Norwegian forest reserves. - Rep. Norwegian
For. Res. Inst. 33.11:455-478.

Jonsson, B.G. 1993: Treefall disturbance, succession, and diversity in boreal forest floor vegetation. - Doct. thesis, 100 pp., Dept. Ecol. Bot., Univ. Umeâ.

Jülich, W. 1984: Kleine Kryptogamenflora 2b(1), Basidiomyceten 1. Die Nichtblätterpilze, Gallertpilze und Bauchpilze (Aphyllophorales, Heterobasidiomycetes, Gastromycetes). - $626 \mathrm{pp}$. Gustav Fischer, Stuttgart \& New York.

Käärik, A. \& Rennerfelt, E. 1957: Investigations on the fungal flora of spruce and pine stumps. - Medd. Stat. Skogsforskningsinst. 47:1-40.

Kaila, L., Martikainen, P., Punttila, P. \& Yakovlev, E. 1994: Saproxylic beetles (Coleoptera) on dead birch trunks decayed by different polypore species. Ann. Zool. Fennici 31:97-107.

Kalela, A. 1961: Waldvegetationszonen Finnlands und ihre klimatischen Paralleltypen. - Arch. Soc. Vanamo 16 (suppl.): 65-83.

Karlsson, J.-O. 1993: Genetic structure of populations of root rot fungi with special emphasis on Heterobasidion annosum. - Doct. thesis, 53 pp. + 5 papers. Dept. Forest Mycol. Pathol., Swedish Univ. Agric. Sci., Uppsala.

Karström, M. 1992a: Steget före - en presentation. Svensk Bot. Tidskr. 86:103-114.

Karström, M. 1992b: Steget före i det gömda landet. Svensk Bot. Tidskr. 86:115-146.

Kay, E. \& Vilgalys, R. 1992: Spatial distribution and genetic relationships among individuals in a natural population of the Oyster mushroom Pleurotus cornucopioides. - Mycologia 84:173-182.

Koponen, T., Isoviita, P. \& Lammes, T. 1977: The bryophytes of Finland: An annotated checklist. Flora Fennica 6:1-77.

Korhonen, K. 1978a: Intersterility groups of Heterobasidion annosum. - Commun. Inst. Forest. Fenniae 94:1-25.

Korhonen, K. 1978b: Interfertility and clonal size in the Armillaria mellea complex. - Karstenia 18:31-42.

Kotiranta, H. \& Niemelä, T. 1981: Composition of the polypore communities of four forest areas in southern Finland. - Karstenia 21:31-48.

Kotiranta, H. \& Niemelä, T. 1993: Uhanalaiset käävät Suomessa. (English summary: Threatened polypores in Finland.) - 116 pp. Vesi- ja ympäristöhallitus, Helsinki.

Kropp, B.R. 1982a: Fungi from decayed wood as ectomycorrhizal symbionts of western hemlock. Canadian J. Forest Res. 12:36-39.

Kropp, B.R. 1982b: Rotten wood as mycorrhizal inoculum for containerized western hemlock. Canadian J. Forest Res. 12:428-431.

Kropp, B.R. \& Trappe, J.M. 1982: Ectomycorrhizal fungi of Tsuga heterophylla. - Mycologia 74:479_ 488.

Lambert, R.L., Lang, G.E., Reiners, W. A. 1980: Loss of mass and chemical change in decaying boles of a subalpine balsam fir forest. - Ecology 61:14601473.

Lange, M. 1986: Fungus succession on fallen logs of beach. - Svampe 13:38-31.

Lange, M. 1992: Sequence of macromycetes on decaying 
beech logs. - Persoonia 14:449-456.

Larsen, M.J., Harvey, A.E., Jurgensen, M.F. 1980: Residue decay processes and associated environmental functions in northern Rocky Mountain forests. - U. S. D. A. For. Service, Gen. Tech. Rep. INT-90:157-174.

Larsson, K.-H. 1986: Wood-inhabiting, resupinate basidiomycetes from Halleberg and Hunneberg in Västergötland, southwest Sweden. - Windahlia 15:23-34.

Lewis, K.J. \& Hansen, E.M. 1991: Vegetative compatibility groups and protein electrophoresis indicate a role for basidiospores in spread of Inonotus tomentosus in spruce forests of British Columbia. Canadian J. Bot. 69:1756-1763.

Lopez, S.E. 1983: Sucesion fungica en madera de Eucalyptus viminalis (Myrtacea) 1. Basidiomycetes sobre arbol vivo y troncos en estiba. - Bol. Soc. Argentina Bot. 22:21-39.

Luschka, N. 1993: Die Pilze des Nationalparks Bayerisher Wald im bayerisch-böhmischen Grenzgebiete. Hoppea, Denkschr. Regensb. Bot. Ges. 53:5-363.

Magurran, A.E. 1988: Ecological diversity and its measurement. - 179 pp. Princeton Univ. Press, Princeton.

Maser, C. \& Trappe, J.M. 1984: The seen and unseen world of the fallen tree. - U. S. D. A. Forest Service, Gen. Tech. Rep. PNW-164:1-56.

Mathiassen, G. 1993: Corticolous and lignicolous Pyrenomycetes s. lat. (Ascomycetes) on Salix along a mid-Scandinavian transect. - Sommerfeltia 20:1-180.

McCullough, H.A. 1948: Plant succession on fallen logs in a virgin spruce-fir forest. - Ecology 29:508-513.

Meredith, D.S. 1959: The infection of pine stumps by Fomes annosus and other fungi. - Ann. Bot. 23:445-476.

Meredith, D.S. 1960: Further observations on fungi inhabiting pine stumps. - Ann. Bot. 24:63-78.

Montgomery, R.A.P. 1982: The role of polysaccharidase enzymes in the decay of wood by basidiomycetes. In: Frankland, C. Hedger, J.N. \& Swift, M.J. (eds.), Decomposer basidiomycetes: their biology and ecology: pp 51-65. - Cambridge Univ. Press, London.

Muhle, H. \& LeBlanc, F. 1975: Bryophyte and lichen succession on decaying logs. 1. Analysis along an evaporational gradient in eastern Canada. - J. Hattori Bot. Lab. 39:1-33.

Niemelä, T. 1994a: Suomen kääpien määritysopas.Helsingin yliop. kasvit. lait. monist. 138:1-131.

Niemelä, T. 1994b: Five species of Anomoporia — rare polypores of old forests. - Ann. Bot. Fennici 31:93-115.

Niemelä, T., Kotiranta, H. \& Penttilä, R. 1992: New records of rare and threatened polypores in Finland. — Karstenia 32:81-94.

Niemelä, T., Renvall, P. \& Penttilä, R. 1995: Interactions of fungi at late stages of wood decomposition. - In: Stokland, J. \& al. (eds.), Biodiversity and management of boreal forests, University of Oslo (submitted).

Nobles, M.K. 1948: Studies in forest pathology 6.
Identification of cultures of wood-rotting fungi. Canadian J. Res. C, 26:281-431.

Nobles, M.K. 1965: Identification of cultures of woodinhabiting Hymenomycetes. - Canadian J. Bot. 43:1097-1139.

Norokorpi, Y. 1979: Old Norway spruce stands, amount of decay and decay-causing microbes in northern Finland. - Comm. Inst. Forest. Fenniae 97:1-66.

Otjen, L. \& Blanchette, R.A. 1986a: A discussion of microstructural changes in wood during decomposition by white rot basidiomycetes. Canadian J. Bot. 64:905-911.

Otjen, L. \& Blanchette, R.A. 1986b: Selective delignification of birch wood (Betula papyrifera) by Hirschioporus pargamenus in the field and laboratory. - Holzforschung 40:183-189.

Park, D. 1968: The ecology of terrestrial fungi. In: Ainsworth, G.C. \& Sussman, A S. (eds.), The fungi, an advanced treatise 3: pp. 5-39. - Academic Press, New York.

Rassi, P., Kaipiainen, H., Mannerkoski, I. \& Ståhls, G. (eds.) 1992: Uhanalaisten eläinten ja kasvien seurantatoimikunnan mietintö. - Komiteamietintö 1991(30):1-328. Ympäristöministeriö, Helsinki.

Rayner, A.D.M. 1977a: Fungal colonization of hardwood stumps from natural sources 1 . Non-basidiomycetes. — Trans. British Mycol. Soc. 69:291-302.

Rayner, A.D.M. 1977b: Fungal colonization of hardwood stumps from natural sources 2. Basidiomycetes. Trans. British Mycol. Soc. 69:303-312.

Rayner, A.D.M. \& Boddy, L. 1988a: Fungal decomposition of wood: its biology and ecology. 587 pp. John Wiley \& Sons, Bath.

Rayner, A.D.M. \& Boddy, L. 1988b: Fungal communities in the decay of wood. - Adv. Microbial Ecol. 10:115-166.

Rayner, A.D.M., Boddy, L. \& Dowson, C.G. 1987: Temporary parasitism of Coriolus spp. by Lenzites betulina: a strategy for domain capture in wood decay fungi. - FEMS Microbiol. Ecol. 45:53-58.

Rayner, A.D.M. \& Hedges, M.J. 1982: Observations on the specificity and ecological role of basidiomycetes colonizing dead elm wood. Trans. British Mycol. Soc. 78:370-373.

Rayner, A.D.M. \& Todd., N.K. 1979: Population and community stucture and dynamics of fungi in decaying wood. - Adv. Bot. Res. 7:333-420.

Renvall, P. 1992: Basidiomycetes at the timberline in Lapland 4. Postia lateritia n. sp. and its rustcoloured relatives. - Karstenia 32:43-60.

Renvall, P. \& Niemelä, T. 1992a: Basidiomycetes at the timberline in Lapland 3. Two new boreal polypores with intricate hyphal systems. - Karstenia 32:2942.

Renvall, P. \& Niemelä, T. 1992b: Fungi on fungi: Wood -rotting Basidiomycetes and their threatened successors. - XI Congress of European Mycologists, Kew, 7-11 September 1992, Abstracts: 35.

Renvall, P. \& Niemelä, T. 1994: Lahoamistavat sienilajiston monimuotoisuutta kaatuneissa puunrungoissa. - Luonnon Tutkija 98:186-193.

Renvall, P., Renvall, T. \& Niemelä, T. 1991a: Basidiomycetes at the timberline in Lapland 1. 
Introduction. - Karstenia 31:1-12.

Renvall, P., Renvall, T. \& Niemelä, T. 1991b: Basidiomycetes at the timberline in Lapland 2. An annotated check-list of the polypores of northeastern Finland. - Karstenia 31:13-28.

Roll-Hansen, F. \& Roll-Hansen, H. 1979: Microflora of sound-looking wood in Picea abies stems. European J. Forest Pathol. 9:308-316.

Runge, A. 1978: Pilzsukzession auf Kiefernstümpfen. —Z. Mykol. 44:295-301.

Runge, A. 1986: Pilzsukzession auf Kiefernstümpfen 2. — Z. Mykol. 52:429-437.

Rypacek, V. 1966: Biologie holzzerstörender Pilze. — 211 pp. Gustav Fischer, Jena.

Ryvarden, L. 1991: Genera of polypores. Nomenclature and taxonomy. - Synopsis Fungorum 5:1-363.

Ryvarden, L. 1994: Notes on some corticioid fungi from Finnmark, northern Norway. - Agarica 13:49-51.

Ryvarden, L. \& Gilbertson, R.L. 1993: European polypores 1. Abortiporus-Lindtneria. - Synopsis Fungorum 6:1-387.

Ryvarden, L. \& Gilbertson, R.L. 1994: European polypores 2. Meripilus-Tyromyces. - Synopsis Fungorum 7:388-743.

Santesson, R. 1993: The lichens and lichenicolous fungi of Sweden and Norway. - 240 pp. SBT-förlaget, Lund.

Siitonen, J. 1994: Decaying wood and saproxylic Coleoptera in two old spruce forests: a comparison based on two sampling methods. - Ann. Zool. Fennici 31:89-95.

Smith, M.L., Bruhn, N. \& Anderson, J.B. 1992: The fungus Armillaria bulbosa is among the largest and oldest living organisms. - Nature 356:428-431.

Söderström, L. 1987: The regulation of abundance and distribution patterns of bryophyte species on decaying logs in spruce forests. - 144 pp. Doct. thesis, Univ. Umeå.

Söderström, L. 1988: Sequence of bryophytes and lichens in relation to substrate variables of decaying coniferous wood in northern Sweden. - Nordic J. Bot. 8:89-97.

Solheim, H. 1992a: The early stages of fungal invasion in Norway spruce infested by the bark beetle Ips typographus. - Canadian J. Bot. 70:1-5.

Solheim, H. 1992b: Fungal succession in sapwood of Norway Spruce by the bark beetle Ips typographus. — Europen J. Forest Pathol. 22:136-148.

Sollins, P. 1982: Input and decay of coarse woody debris in coniferous stands in western Oregon and Washington. - Canadian J. Forest Res. 12:18-28.

Sollins, P., Cline, S.P., Verhoeven, T., Sachs, D. \& Spycher, G. 1987: Patterns of log decay in oldgrowth Douglas-fir forests. - Canadian J. Forest Res. 17:1585-1595.

Stalpers, J.A. 1978: Identification of wood-inhabiting Aphyllophorales in pure culture. - Stud. Mycol.
16:1-248.

Stalpers, J.A. 1993: The aphyllophoraceous fungi 1. Keys to the species of the Thelephorales. - Stud. Mycol. $35: 1-168$.

Stenlid, J. 1985: Population structure in Heterobasidion annosum as determined by somatic incompatibility, sexual compatibility, and isoenzyme patterns. Canadian J. Bot. 63:2268-2273.

Stenlid, J. 1993: Community and population biology of boreal decay fungi. In: Pegler, D. N. \& Boddy, L., Ing, B. \& Kirk, P. M. (eds.), Fungi of Europe: Investigation, recording and conservation: pp. 171180. - Royal Botanic Gardens, Kew.

Strid, A. 1975: Wood-inhabiting fungi of alder forests in North-Central Scandinavia 1. Aphyllophorales (Basidiomycetes). Taxonomy, ecology and distribution. - Wahlenbergia 1:1-237.

Swift, M.J. 1977: The ecology of wood decomposition. Sci. Prog. Oxford 64:179-203.

Swift, M. J. 1982: Basidiomycetes as components of forest ecosystems. In: Frankland, J.C., Hedger, J.N. \& Swift, M.J. (eds.), Decomposer basiodiomycetes: their biology and ecology: pp. 307-337. Cambridge Univ. Press, Cambridge etc.

Swift, M.J. 1987: Organization of assemblages of decomposer fungi in space and time. In: Gee, J.H.R. \& Giller, P.S. (eds.), Organization of communities past and present: pp. 519-542. - Blackwell, Oxford etc.

Tanesaka, E., Masuda, H. \& Kinugawa, K. 1993: Wood degrading ability of basidiomycetes that are wood decomposers, litter decomposers, or mycorrhizal symbionts. - Mycologia 85:347-354.

Thompson, W. \& Boddy, L. 1983: Decomposition of suppressed oak trees in even-aged plantations 2. Colonization of tree roots by cord- and rhizomorphproducing basidiomycetes. - New Phytol. 93:277291.

Todd, N.K. \& Rayner, A.D.M. 1980: Fungal individualism. - Sci. Prog. Oxford 66:331-354.

Virkkala, R., Rajasärkkä, A., Väisänen, R.A., Vickholm, M. \& Virolainen, E. 1994: Conservation value of nature reserves: do hole-nesting birds prefer protected forests in southern Finland? - Ann. Zool. Fennici 31:173-186.

Vogt, K.A., Bloomfield, J., Ammirati, J.F. \& Ammirati, S.R. 1992: Sporocarp production by Basidiomycetes, with emphasis on forest ecosystems. In: Wicklow, D.T. \& Carroll, C.G. (eds.), The fungal community. Its organization and role in the ecosystem: pp. 563581. - Marcel Dekker, New York.

Zackrisson, O. 1977: Influence of forest fires on the north Swedish boreal forest. — Oikos 29:22-32.

Received on 9 March 1995 
